\title{
Ambipolar Ballistic Electron Emission Microscopy Studies of Gate-field Modified Schottky Barriers
}

\author{
Dissertation \\ By \\ Yulu Che, M.S. \\ Graduate Program in Physics \\ The Ohio State University \\ 2010 \\ Dissertation Committee: \\ Jonathan P. Pelz, Advisor \\ Nandini Trivedi \\ Gregory Lafyatis \\ Fengyuan Yang
}

Presented in Partial Fulfillment of the Requirements for the Degree Doctor of Philosophy in the Graduate School of The Ohio State University 


\section{Copyright by}

\section{Yulu Che}

2010 


\begin{abstract}
In this thesis, we present two separate studies: (1) The development and use of "amibipolar" ballistic electron emission microscopy (BEEM), which allows the measurement of Schottky barrier heights (SBHs) for both electrons and holes at the same location on the same metal/semiconductor contact with nm-scale spatial resolution and meV-scale energy resolution. A significant accomplishment of this study is the successful development of a new method that can monitor lateral variations of the local interface electric field and local interface dipoles at metal/semiconductor contacts by monitoring local variations in the electron- and hole SBHs. (2) Ion milling and inductively coupled plasma reactive ion etching (ICP-RIE) of vertical silicon nanowires, in order to increase the fraction of vertical vs. non-vertical nanowires.
\end{abstract}

In the first experiment, novel four-terminal back-gated Schottky contact sample structures (similar in ways to field effect transistors) were fabricated from 
silicon-on-insulator (SOI) substrates and used to successfully measure the Schottky barrier heights for both electrons and holes at the same location on the same metal/semiconductor $(\mathrm{M} / \mathrm{S})$ contact by using a four-terminal BEEM technique. This measurement cannot be done using conventional three-terminal BEEM on regular metal/bulk silicon contacts, which do not allow the interface electric field under the M/S contact to be modified by an applied gate voltage. With our back-gated Schottky diode structures, by applying a back-gate voltage to the SOI substrate, the Si film could be made either effectively n-type or p-type depending on the polarity of the gate voltage.

Using this gated SOI sample structure, we demonstrated ambipolar BEEM measurements in which BEEM and BHEM (ballistic hole emission microscopy) measurements were performed at the same location on the same $\mathrm{Au} / \mathrm{Si}$ and $\mathrm{Cu} / \mathrm{Si}$ contacts at $\mathrm{T}=80 \mathrm{~K}$. After accounting for image force lowering (IFL), the local Schottky barrier heights for electrons and holes sum to approximately the silicon band gap, indicating that we can accurately probe both the conduction band and valence bands at the same location. Hence, we demonstrated that this technique is sensitive to local variations in the metal/Si interface dipole and/or the interface electric field. While the individual $n$ - and p-SBHs varied by more than $200 \mathrm{meV}$ between the $\mathrm{Au} / \mathrm{Si}$ and $\mathrm{Cu} / \mathrm{Si}$ contacts, for a given sample they sum to within 15 $\mathrm{meV}$ of the same value, indicating that the individual variations are due to 
variations in a local surface dipole as compared with tip effects or variations in local composition.

The second study presents a method to increase the ratio of vertical silicon nanowires (grown by chemical vapor deposition (CVD) with dispersed colloidal $\mathrm{Au}$ particles as catalyst) by ion milling and RIE etching. By choosing the correct ion milling thickness of silicon, we found that it is possible to remove most or all of the non-vertical silicon nanowires leaving mostly or only vertical silicon nanowires standing on the substrate. This is an important achievement which opens up a new opportunity for researches on high density vertical nanowires. Our method may be useful for studies that require samples with a large fraction of vertical nanowires. 
Dedicated to my parents and my wife 


\section{ACKNOWLEDGEMENTS}

First, I would like to give my sincere appreciation to my advisor, Prof. Jonathan P. Pelz, for his guidance and support during my graduate study at OSU. It has been a great pleasure to work with him and I have learned from him a lot about physics. I want to thank Brian Gibbons and Camelia Marginean for teaching me how to use our equipments. I also want to thank our lab members, Kibog Park, Wei Cai, Steve Stone, Jonas Beardsley, Drew Cardwell, Kenji Oman and Austen Rau for their help. I want to thank Tom Kelch, Bob Merritt, Denis Pelekhov, Rita Rokhlin, Iulian Hetel, and the people in the machine shop and the electronic shop for all their technical support. And I want to thank Robert Davis, Paul Steven, Derek Ditmer, Aimee Bross, John Calin and Nirut Pussadee for their support and help in Nanotech West cleanroom. I want to thank Jim Jones for his help in EE cleanroom. I also want to thank Yuri Obukhov and Kin Chung Fong for their help.

I want to thank my parents for their support and encouragement. Finally, I would like to express my heartfelt appreciation to my wife, Huichao Song, for her love, support, unlimited patience, understanding and encouragement. 


\section{VITA}

April 16, 1974..........................Born in Huadian, Jilin, China

1992 - 1996............................. Physics, Jilin University, China

1996 - 1999............................... Physics, Jilin University, China

2002 - Present..........................The Ohio State University

\section{PUBLICATIONS}

1. Y.L.Che and J.P. Pelz, "Ambipolar ballistic electron emission microscopy studies of gate-field modified Schottky barriers", Applied Physics Letter 96, 242106 (2010)

\section{FIELD OF STUDY}

Major Field: Physics 


\section{TABLE OF CONTENTS}

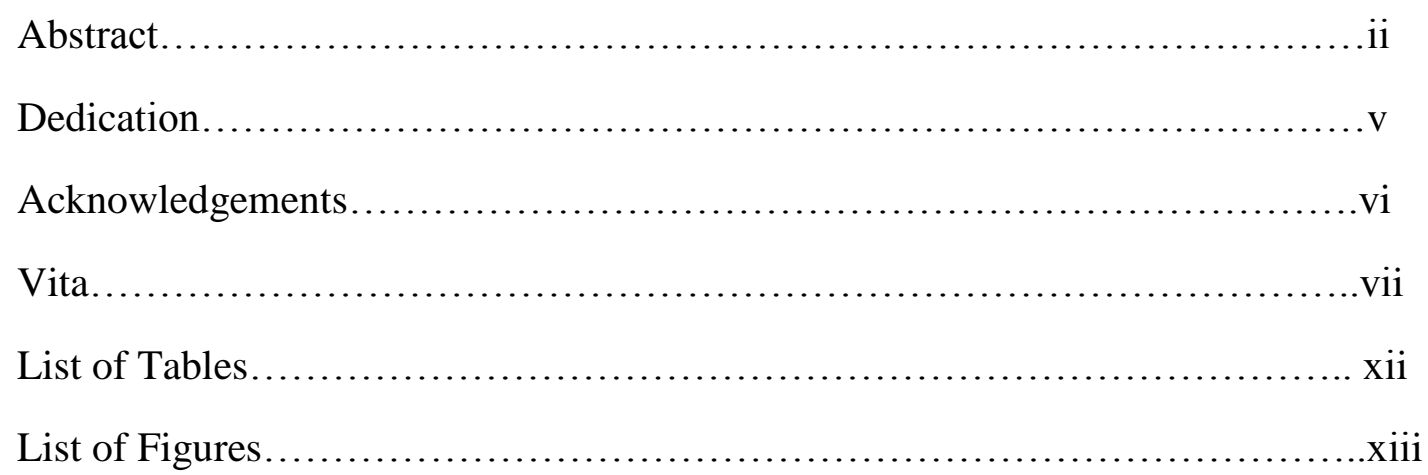

\section{Chapters:}

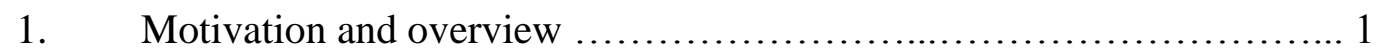

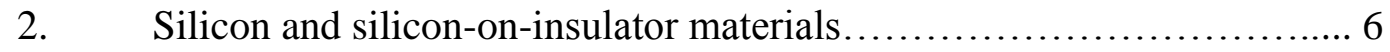

2.1 Silicon.............................................. 6

2.1.1 Crystal structure............................... 6

2.1.2 Energy bands .............................. 9

2.1.3 Carrier concentration at thermal equilibrium..........13

2.1.4 Carrier transport................................. 16 
2.1.5 $\mathrm{SiO}_{2}$ and $\mathrm{Si} / \mathrm{SiO}_{2}$ interface..................... 17

2.2 Silicon-on-insulator.........................................21

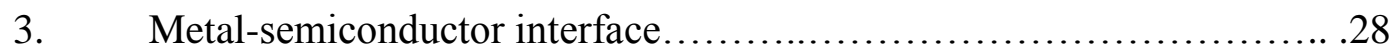

3.1 Schottky barriers ......................................... 28

3.2 Fermi level inning...........................................

3.3 Image force lowering ..................................... 34

3.4 Current transport........................................ 37

3.5 Ohmic contacts..................................................40

3.6 Tung's model- Inhomogeneous Schottky barriers................40

3.7 Characterization of Schottky barrier height...................44

4. Ballistic Electron Emission Microscopy...............................45

4.1 Schematics of BEEM........................................45

4.2 BEEM spectrum.......................................48

4.3 Theoretical models.........................................49

4.4 Equipment setup......................................58

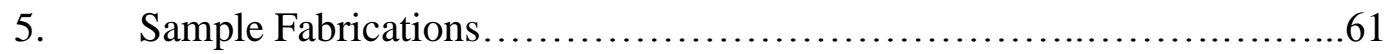

5.1 Sample structure........................................61

5.2 Chemical cleaning...................................... 64

5.2.1 Three-solvent cleaning............................65

5.2.2 Piranha cleaning.................................66

5.2.3 Four-chemical cleaning........................... 66

5.3 Photolithography....................................... 68 
5.3.1 Lift-off with dual-layer resists..................69

5.3.2 Lift-off with single-layer resist................ 71

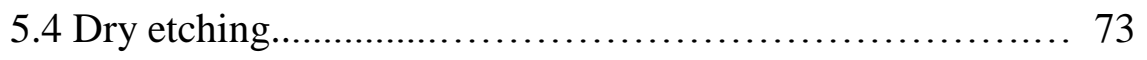

5.4.1 Plasma etching................................................. 73

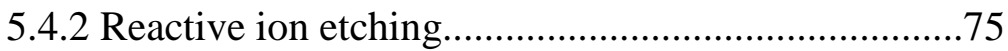

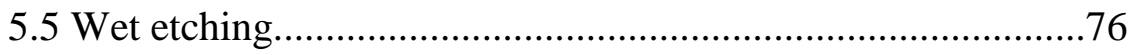

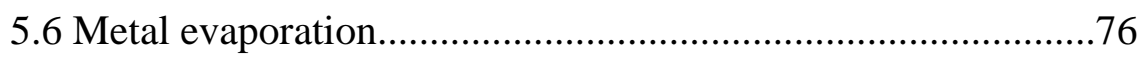

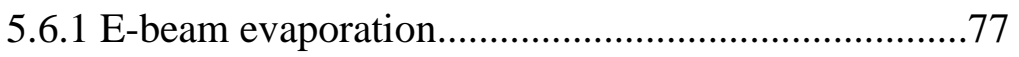

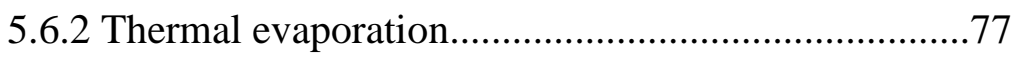

5.7 Fabrication steps for one complete SOI sample....................78

6. Ambipolar BEEM measurements................................80

6.1 Previous BEEM results on $\mathrm{Au} / \mathrm{Si}$ and $\mathrm{Cu} / \mathrm{Si}$ contacts..........81

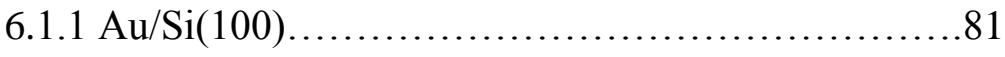

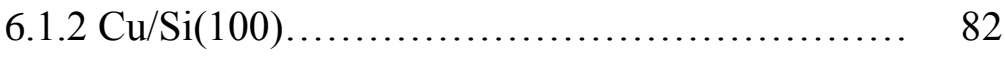

6.2 Ambipolar BEEM studies...........................82

6.2.1 Basic idea of ambipolar BEEM...................86

6.2.2 Ambipolar BEEM measurement results............89

6.3 Explanation of the applied gate voltages..................96

6.4 Image force lowering measurements.................. 104

6.5 Wet etch SOI silicon film......................... 106

6.6 Conclusions and future work $\ldots \ldots \ldots \ldots \ldots \ldots \ldots \ldots \ldots \ldots, 112$

7. Ion milling vertical silicon nanowires........................... 114

7.1 Introduction........................................114 
7.2 Vertical silicon nanowire fabrications....................115

7.3 Ion mill silicon nanowires.............................118

7.4 RIE etch silicon nanowires............................ 124

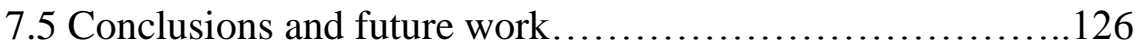

Appendices.......................................................... 127

A Gate voltages for BEEM and BHEM measurements................130

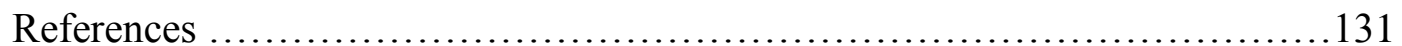




\section{LIST OF TABLES}

$\begin{array}{lll}\text { Table Page } & \text { Pag }\end{array}$

2.1 Physical and electrical properties of modern commercial SIMOX....... 27

5.1 The recipe for plasma etching 35nm SIMOX SOI wafers............. 74 


\section{LIST OF FIGURES}

Figure Page

2.1 Diamond lattice structure where a is the lattice constant............. 7

$2.2 \quad \mathrm{sp}^{3}$ hybrid orbitals.......................................... 7

2.3 Brillouin zone for the diamond lattice........................... 8

2.4 Energy bands in silicon along [111] and [100] directions............. 10

2.5 Constant-energy surface near the conduction band minima........... 11

2.6 Temperature dependence of the energy band gap of silicon.......... 12

2.7 Energy levels for silicon with donor and acceptor impurities..........14

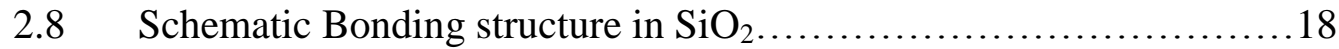

$2.9 \quad$ Amorphous $\mathrm{SiO}_{2}$ structure................................... 19

xiii 
2.10 Terminology of charges associated with thermal $\mathrm{SiO}_{2} \ldots \ldots \ldots \ldots \ldots .21$

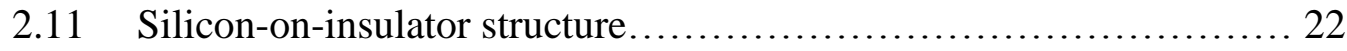

2.12 The basic SIMOX process........................................ 23

2.13 Cross-section TEM images of SIMOX samples before and after HTA at $1360{ }^{\circ} \mathrm{C}$ for 6hours.................................. 23

2.14 Oxygen precipitation, growth and precipitate coalescence during SIMOX annealing with different oxygen doses............. 24

2.15 A set of TEM micrographs shows the existence of an optimum dose for the formation of Si island free BOX layer................. 26

3.1 The energy band diagram of metal-semiconductor contacts.......... 29

3.2 Experimental results of barrier heights for metal-semiconductor Contacts.......................................................... 31

3.3 Energy band diagram with interface states......................... 32

3.4 Energy band diagram between a metal and a vacuum................. 35

3.5 Theoretical and experimental IVs for Au/Si barrier.................. 38

3.6 Low barrier height and/or high doping for ohmic contacts............ 41

3.7 Conduction band minimum potential in an n-type semiconductor near a circular patch................................................ 43

4.1 Schematic representation and Energy band diagram of BEEM........ 46 
4.2 Typical BEEM spectrum on Au/n-Si...........................48

4.3 Schematic energy diagram of the tunneling process..................51

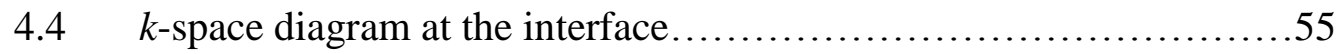

4.5 Schematic of our Omicron variable temperature STM system.........59

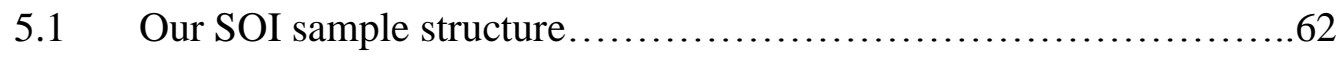

5.2 Lift-off process with dual-layer resists..............................70

5.3 Lift-off process with single-layer resist.......................... 72

6.1 BEEM spectrum measured on $\mathrm{Au} / \mathrm{n}-\mathrm{Si}(100)$ and $\mathrm{Au} / \mathrm{p}-\mathrm{Si}(100) \ldots \ldots \ldots 83$

6.2 Energy band diagrams of metal $/ \mathrm{n}-\mathrm{Si}$ and metal $/ \mathrm{p}-\mathrm{Si} \ldots \ldots \ldots \ldots \ldots \ldots . \ldots 5$

6.3 Schematic diagram of sample geometry and wiring for ambipolar BEEM measurements......................................... 87

6.4 Energy band structure for $\mathrm{Au} / \mathrm{Si}$ barriers ........................... 88

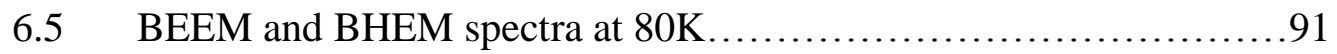

6.6 Energy band diagram for positive and negative substrate bias.........98

6.7 COMSOL simulation without interface states........................100

6.8 COMSOL simulation on Au/SOI with interface states................101

6.9 COMSOL simulation on $\mathrm{Cu} / \mathrm{SOI}$ with interface states................102

6.10 Energy band diagram when there is a bias between the Schottky

Contact and the ohmic contact..................................104 
6.11 Schottky barrier heights versus bias on Schottky contacts........... 105

6.12 SOI sample structure with sharp corners...................... 107

6.13 AFM images of the Si surface before and after SC-1 etching.........109

6.14 AFM images of $\mathrm{Si}$ surface etched with $\mathrm{SiO}_{2}$ mask............... 110

6.15 AFM images of etched step on SOI wafer.......................111

7.1 SEM images of vertical Si nanowires grown by VLS mechanism.....116

7.2 SEM images of vertical Si nanowires etched with ICP-RIE..........117

7.3 Dual beam FIB/SEM.......................................... 119

7.4 SEM image of nanowires....................................... 120

7.5 SEM image of nanowires after 10nm ion milling ...................121

7.6 SEM image of nanowires after 30nm ion milling ..................122

7.7 SEM image of nanowires after 200nm ion milling..................123

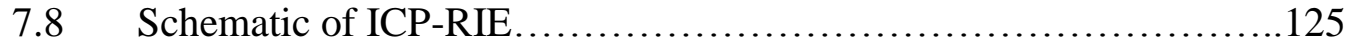

A.1 Geometry for COMSOL numerical simulation....................130 


\section{CHAPTER 1}

\section{MOTIVATION AND OVERVIEW}

In this work, we present the results of two different studies: (1) amibipolar ballistic electron emission microscopy (BEEM) studies on gated silicon-on-insulator (SOI) sample, and (2) ion milling of silicon nanowires to increase the yield of vertical nanowires. The main accomplishments of the first study are: (1) The successful development of a new method that can monitor the energy (relative to the metal Fermi energy) of both the conduction band minimum and the valence band maximum of a thin semiconductor film at the same location. (2) By comparing these two energies, this technique can be used as a method to monitor and quantify lateral variations of (i) the local interface electric field, (ii) local interface dipoles, and (iii) the local bandgap at metal/semiconductor contacts. The main accomplishment of the second study is the development of a method to increase the ratio of vertical silicon nanowires that are grown epitaxially on a Si substrate. 


\section{Amibipolar ballistic electron emission microscopy (BEEM) studies on}

gated silicon-on-insulator (SOI) sample. There is an increasing interest in nm-size electronic device structures in which current flow is controlled by internal tunnel barriers that are modified by an applied gate bias. For example, it has been argued that carbon nanotube field effect transistors (FETs) [1] can behave as Schottky barrier FETs [2] in which the current is controlled by gate-field modulated tunneling through a Schottky barrier. One characteristic of Schottky barrier FETs is ambipolar transport [2], in which either electrons or holes are the primary carriers that tunnel through a gate-field controlled tunnel barrier. Gate-field controlled tunneling is also the basis of Tunneling FETs [3,4], which have been proposed to have better scalability and demonstrated to have a lower sub-threshold swing than possible in conventional FETs. Since locally-enhanced electric fields (for example at the edges of a gate or the channel region) can strongly impact the local tunneling in Schottky barrier and Tunneling FETs, it is important to be able to measure the effects of an applied field on the local barrier properties for both electron and hole transport. However, how the Schottky barriers are modified by the gate field can't be determined by conventional methods such as $I_{S D}$ (source-drain current) versus $V_{G}$ (gate voltage) measurement. For example, in carbon nanotube FETs, the gate voltage affects the source-drain current by modulating both the nanotube channel conductance and the Schottky barriers at the source/drain end, so it is difficult to extract the effect of the gate voltage on the Schottky barrier from $I_{S D}-V_{G}$ 
measurement. In this study, we take advantage of the high spatial resolution (nmscale) and high energy precision (several $\mathrm{meV}$ ) of the technique of BEEM and modified it from a three-probe technique to a four-probe technique. By applying this modified BEEM technique on a novel sample structure fabricated on SOI wafers, we succeeded in varying the electric-field under the Schottky contacts by applying a gate voltage and measuring how the Schottky barriers are modified by this electricfield.

II. Ion milling of vertical Si nanowires. This study presents a method to increase the yield of vertical silicon nanowires by ion milling. Vertical silicon nanowires have been used to fabricate some novel devices such as silicon vertically integrated nanowire FETs or Surround-gate FETs silicon nanowire photodetectors, and silicon nanowire solar cell. These devices were claimed to have some better performance than conventional structures. And end-on metal contacts to vertical Si nanowires can also be used to study the role of Fermi level pinning in nano-scale metal-semiconductor contacts, which is one of the critical issues in nanowire or carbon nanotube FETs. Our method may be found useful by someone who wants to conduct research on vertical Si nanowires but has difficulty in obtaining samples that do not have a high density of vertical nanowires.

Background information and experimental results of our work are presented in the following chapters: 
Chapter 2 is an introduction to silicon and silicon-on-insulator properties. Several topics important to our studies are covered: energy bands of silicon, carrier concentration at thermal equilibrium, $\mathrm{Si} / \mathrm{SiO}_{2}$ interface, and structure and fabrication of SIMOX (Separation by IMplantation of Oxygen) SOI.

Chapter 3 covers several concepts related to Schottky barriers: formation of Schottky barriers, Fermi level pinning, image force lowering, inhomogeneity of the Schottky barriers, and ohmic contacts.

In Chapter 4, the technique of BEEM is described in detail. The basic idea of BEEM and theoretical models for extracting barrier heights are presented.

Chapter 5 describes the preparation methods of our samples used in our measurements, including chemical cleaning, photolithography, dry-etching, and metal evaporation, etc..

In Chapter 6, we demonstrate our ambipolar BEEM measurements in which BEEM and BHEM measurements were performed at the same location on the same $\mathrm{Au} / \mathrm{Si}$ and $\mathrm{Cu} / \mathrm{Si}$ contacts at $\mathrm{T}=80 \mathrm{~K}$. After accounting for image force lowering, the local SBHs for electrons and holes sum to approximately the Si bandgap, indicating that we can accurately probe both the conduction band and valence bands at the same location. While the individual $n$ - and p-SBHs varied by more than $200 \mathrm{meV}$ between the $\mathrm{Au} / \mathrm{Si}$ and $\mathrm{Cu} / \mathrm{Si}$ contacts, for a given sample they sum to within 15 $\mathrm{meV}$ of the same value, indicating that the individual variations are due to variations 
in a local surface dipole as compared with tip effects or variations in local composition.

Chapter 7 is our results on using ion milling to increase the yield of vertical silicon nanowires. We found that the fraction of vertical NWs can be increased, but some damage does occur to the remaining (vertical) NWs if the milling is done too long. By choose the correct ion milling thickness of silicon, all of the non-vertical silicon nanowires can be removed leaving only vertical silicon nanowires standing on the substrate. 


\section{CHAPTER 2}

\section{SILICON AND SILICON-ON-INSULATOR MATERIALS}

\subsection{Silicon \\ In this section, some properties of $\mathrm{Si}$ and $\mathrm{Si} / \mathrm{SiO}_{2}$ interfaces will be discussed.}

\subsubsection{Crystal structure}

Silicon belongs to Group IV A and has an atomic configuration of $[\mathrm{Ne}] 3 \mathrm{~s}^{2} \mathrm{p}^{2}$ [5]. The crystal structure of silicon is the diamond lattice structure in which each silicon atom is surrounded by four equidistant nearest silicon atoms which lie at the corners of a tetrahedron, and the lattice constant is a $=5.43 \AA$, as shown in Figure 2.1 [6]. In crystalline silicon, the four outermost shell electrons $\left(3 \mathrm{~s}^{2} 3 \mathrm{p}^{2}\right)$ form four equivalent $\mathrm{sp}^{3}$ hybrid orbitals which are directed at an angle of $109.5^{\circ}$ relative to each other as shown in Figure 2.2 [6]. The bond between two nearest silicon 


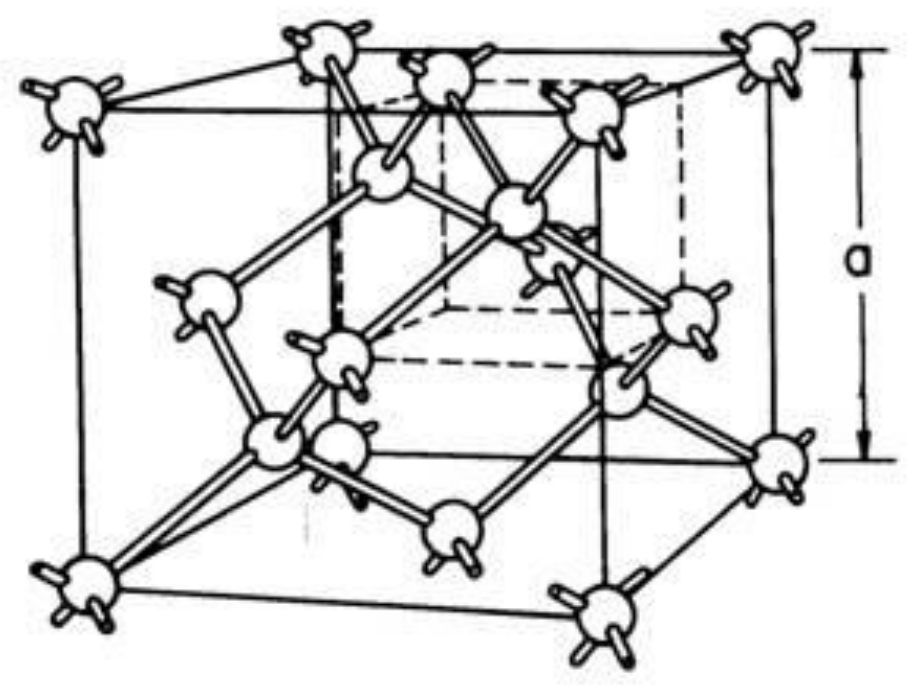

Figure 2.1: Diamond lattice structure where $\mathbf{a}$ is the lattice constant (figure taken from Ref. [6] ).

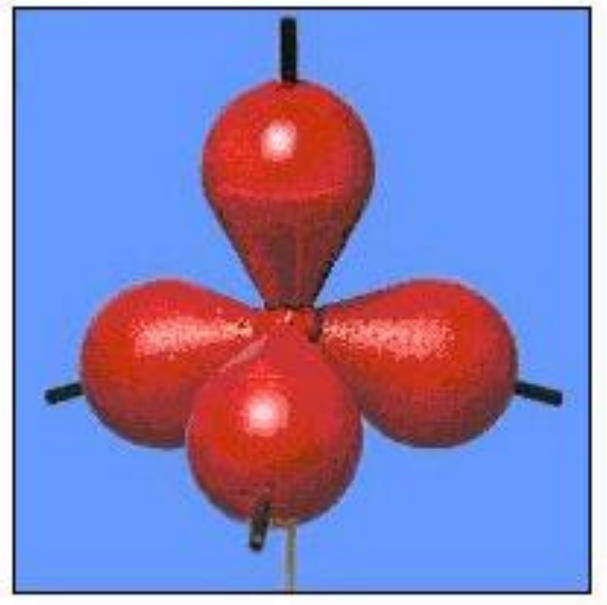

Figure 2.2: $\mathrm{sp}^{3}$ hybrid orbitals (figure taken from Ref. [7]) . 
atoms is a covalent $\boldsymbol{\sigma}$ bond formed by head-to-head overlapping of two $\mathrm{sp}^{3}$ hybrid orbitals with opposite spins.

Since the diamond lattice can be considered as two interpenetrating facecentered cubic (FCC) lattices which are shifted by $\sqrt{3} a / 4$ along the diagonal [5], the Bravais lattice of silicon crystal is the FCC lattice and the reciprocal lattice is the body-centered cubic (BCC) lattice. The first Brillouin zone for the diamond lattice is the truncated octahedron as shown in Figure 2.3 in which the most important symmetry points and symmetry lines are indicated: zone center $\Gamma$ : $2 \pi / \mathrm{a}(0,0,0) ; \mathrm{X}: 2 \pi / \mathrm{a}(0,0,1) ; \mathrm{L}: 2 \pi / \mathrm{a}(1 / 2,1 / 2,1 / 2) ; \mathrm{K}: 2 \pi / \mathrm{a}(3 / 4,3 / 4,0) ; \Delta:\langle 100>$ axes; $\Sigma:\langle 110>$ axes; and $\Lambda:<111>$ axes [4].

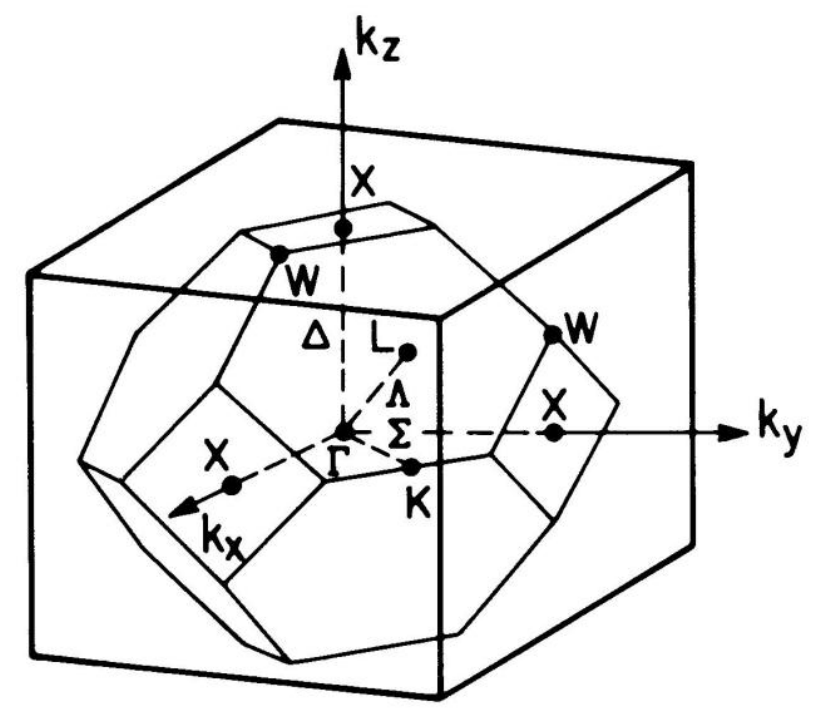

Figure 2.3: Brillouin zone for the diamond lattice (figure taken from Ref. [8]). 


\subsubsection{Energy bands}

The energy band structure describes the relationship between the energy $E$ and the momentum $\boldsymbol{k}$ which can be obtained by solving the Schrödinger equation with the aid of group theory [9]. Figure 2.4 shows the energy bands in silicon along the [100] and [111] directions [10]. The upper part shows the conductions bands and the lower part shows the valence bands. The separation is the silicon band gap $E_{g}=1.12 \mathrm{eV}$ at room temperature. The lowest conduction band has a minimum at a point along the [100] direction. For the valence bands, two degenerate bands meet at $\Gamma(0,0,0)$, giving the maximum of the valence bands. These two valence bands have different curvature at point $\Gamma$ and are called the light-hole band and the heavyhole band respectively. The third valence band is separated from the other two bands by roughly $0.044 \mathrm{eV}$ because of the spin-orbital coupling. Since the conduction band minimum and the valence band maximum occur at different points in $\boldsymbol{k}$-space, silicon is an indirect band-gap semiconductor.

At the conduction band minimum $\left(k_{x}^{\min }, k_{y}^{\min }, k_{z}^{\min }\right)$, the energy of electron can be written as

$$
E(\vec{k})=E_{C}+\frac{\hbar^{2}}{2}\left(\frac{\left(k_{x}-k_{x}^{\min }\right)^{2}}{m_{x}}+\frac{\left(k_{y}-k_{y}^{\min }\right)^{2}}{m_{y}}+\frac{\left(k_{z}-k_{z}{ }^{\min }\right)^{2}}{m_{z}}\right)
$$




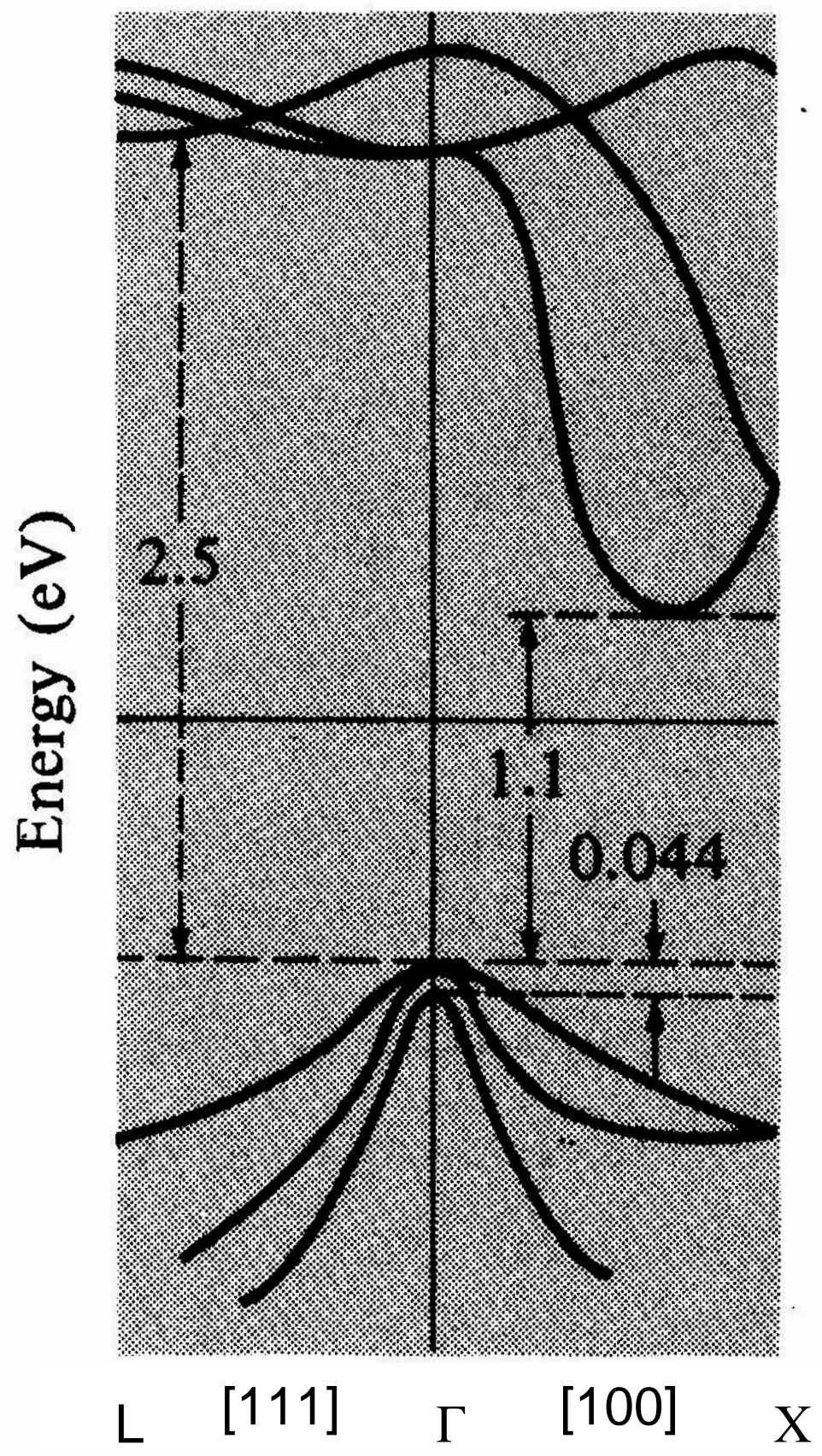

Figure 2.4: Energy bands in silicon along [111] and [100] directions (figure taken from Ref. [10]). 


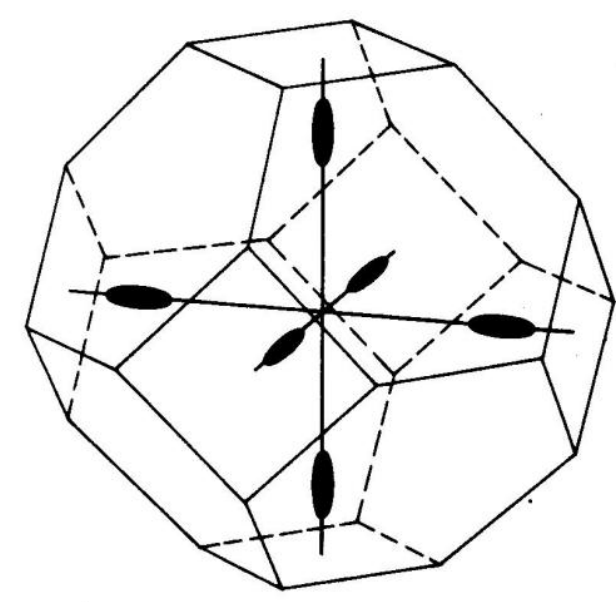

Figure 2.5: Constant-energy surface near the conduction band minima (figure taken from Ref. [5]).

where $E_{C}$ is the energy at the bottom of the conduction band, and $m_{x}, m_{y}$ and $m_{z}$ are the effective masses of electrons along $\mathrm{x}, \mathrm{y}$ and $\mathrm{z}$ axis. We can see the constant energy surfaces are ellipsoidal in shape and for silicon there are six ellipsoids along the $<100>$ axes due to the symmetry of the first Brillouin zone as shown in Figure 2.5 [5]. The effective mass along the $<100>$ axes (the longitudinal effective mass) is $m_{l}^{*}=0.98 m_{0}$, and the effective mass perpendicular to the $\langle 100\rangle$ axes (the transverse effective mass) is $m_{t}^{*}=0.19 m_{0}$, where $m_{0}=0.911 \times 10^{-30} \mathrm{~kg}$ is the free electron mass [5]. At the valence band maximum the two degenerate valence bands are spherically symmetric and the energy can be written as 


$$
E(\vec{k})=E_{V}-\frac{\hbar^{2}}{2} \frac{k_{x}^{2}+k_{y}^{2}+k_{z}^{2}}{m_{h}}
$$

where $E_{V}$ is the maximum energy of the valence bands, $m_{h}$ is the effective mass of holes which is equal to $0.16 m_{0}$ for the light-hole band and $0.49 m_{0}$ for the heavyhole band [5].

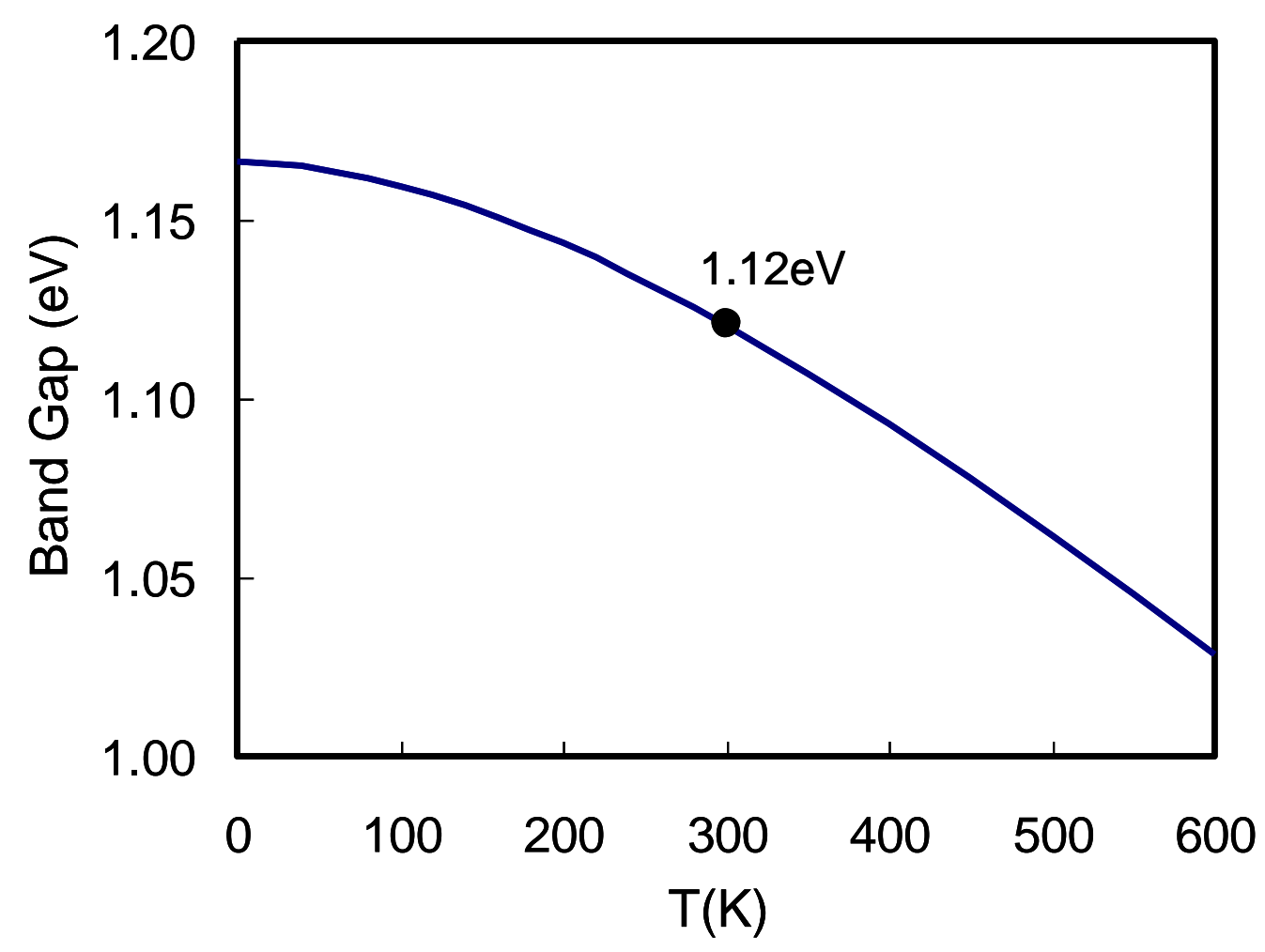

Figure 2.6: Temperature dependence of the energy band gap of silicon. 
As for most semiconductors, the energy band gap of $\mathrm{Si}$ decreases with increasing temperature and has a temperature dependence of

$$
E_{g}(T)=E_{g}(0)-\alpha T^{2} /(T+\beta)
$$

as shown in Figure 2.6, where $E_{g}(0)=1.170 \mathrm{eV}$ is the $\mathrm{Si}$ band gap at $T=0 \mathrm{~K}, \alpha=$ $4.83 \times 10^{-4} \mathrm{eV} / \mathrm{K}$ and $\beta=636 \mathrm{~K}[6]$. This temperature dependence is due to the thermal expansion of the periodic crystal lattice and the effect of lattice vibration on the band structure and energy band gap [5].

\subsubsection{Carrier concentration at thermal equilibrium}

For intrinsic (undoped) silicon at zero temperature, the conduction band is empty and the valence band is fully filled with electrons. When $T>0 \mathrm{~K}$, some electrons will be thermally excited from the valence band to the conduction band which leaves an equal number of holes in the valence band, so the free electron density $n$ and the free hole density $p$ are equal, $n=p=n_{i}$ where $n_{i}$ is the intrinsic carrier density which is $\sim 1.45 \times 10^{10} / \mathrm{cm}^{3}$ at room temperature for silicon [6]. Using the Fermi-Dirac distribution, the electron density in the conduction band and the hole density in the valence band are given by

$$
n=N_{C} \frac{2}{\sqrt{\pi}} F_{1 / 2}\left(\frac{E_{F}-E_{C}}{k_{B} T}\right)
$$




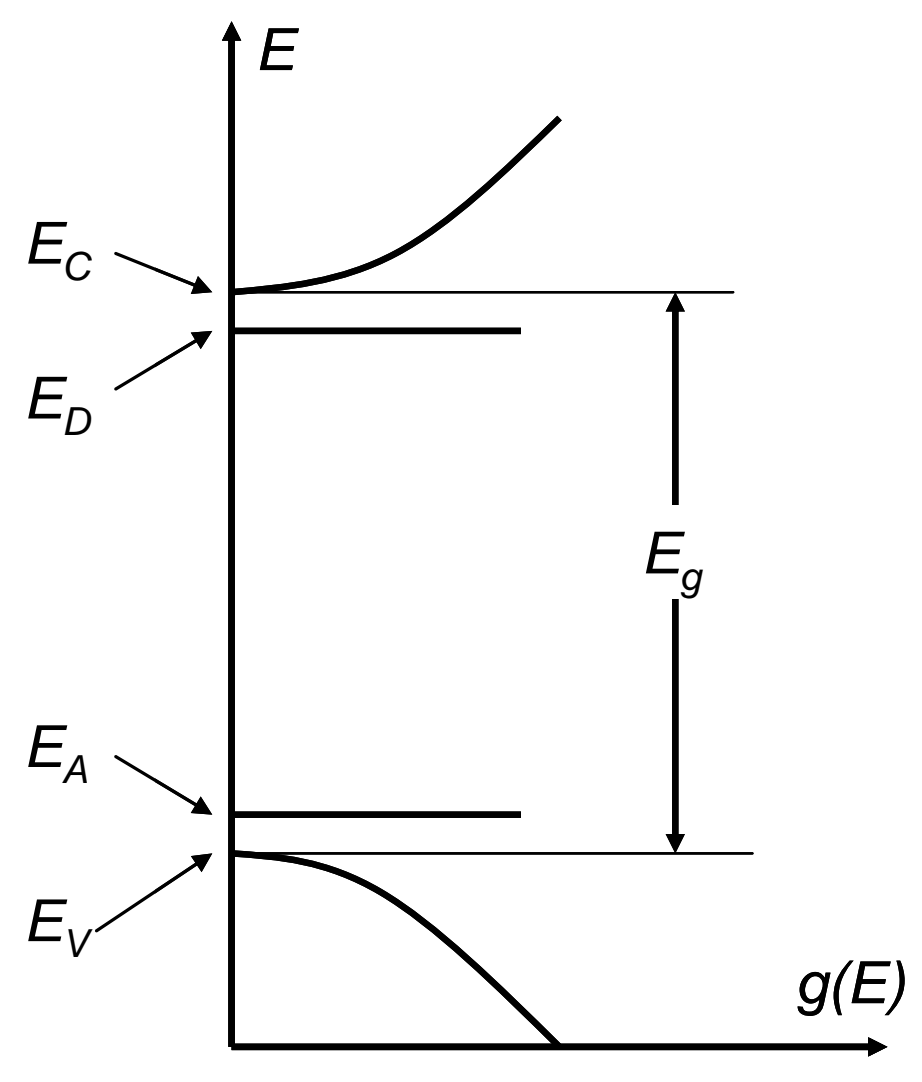

Figure 2.7: Energy levels for silicon with donor and acceptor impurities.

$$
p=N_{V} \frac{2}{\sqrt{\pi}} F_{1 / 2}\left(\frac{E_{V}-E_{F}}{k_{B} T}\right)
$$


where $N_{C}\left(N_{V}\right)$ is the effective density of states in the conduction (valence) band (at room temperature, $N_{C}=2.8 \times 10^{19} / \mathrm{cm}^{3}$, and $\left.N_{V}=1.04 \times 10^{19} / \mathrm{cm}^{3}\right)$, $F_{1 / 2}(x)=\int_{0}^{\infty} d y \sqrt{y} /(1+\exp (y-x))$ is the Fermi-Dirac integral, and $E_{F}$ is the Fermi energy which can be determined by $n=p$. At thermal equilibrium, $n p=n_{i}^{2}=N_{C} N_{V}$ $\exp \left(-E_{g} / k_{B} \mathrm{~T}\right)$, which is called the law of mass action.

In doped silicon, donor or acceptor impurities will introduce impurity level inside the band gap as shown in Figure 2.7, where $E_{D}\left(E_{A}\right)$ is the donor (acceptor) impurity level, which is equal to $E_{C}-0.044 \mathrm{eV}\left(E_{V}+0.046 \mathrm{eV}\right)$ for Phosphorus (Boron) in silicon. At $\mathrm{T}=0 \mathrm{~K}$, a donor (acceptor) level is occupied by an electron (a hole) and is neutral. At elevated temperatures, the electron (hole) at a donor (acceptor) level will be excited to the conduction (valence) band, so the density of electrons (holes) in the conduction (valence) band is different from $n_{i}$, but can still be evaluated by Equation (2.4) (Equation (2.5)). However, in this case the Fermi energy $E_{F}$ is not the same as it is in intrinsic silicon, and should be determined by the neutrality condition

$$
n+N_{A}^{-}=p+N_{D}^{+}
$$

In the above equation (2.6), $N_{A}{ }^{-}\left(N_{D}{ }^{+}\right)$is the ionized donor (acceptor) density 


$$
N_{A}^{-}=\frac{N_{A}}{1+4 \exp \left(\left(E_{A}-E_{F}\right) / k_{B} T\right)}
$$

and

$$
N_{D}^{+}=\frac{N_{D}}{1+2 \exp \left(\left(E_{F}-E_{D}\right) / k_{B} T\right)}
$$

where $N_{A}\left(N_{D}\right)$ is the donor (acceptor) density and the number 4(2) in the denominator is the degeneracy factor of the acceptor (donor) levels[6].

\subsubsection{Carrier transport}

The basic equations which describe the carrier transport phenomena in semiconductors are the Maxwell equations, the current-density equations, and the continuity equation, which are discussed in detail in section 1.5 of Reference.6. Here, the three equations which are used most frequently in semiconductor device simulations are listed below.

(1) The Poisson equation:

$$
\nabla \cdot(-\varepsilon \nabla \psi)=q\left(p+N_{D}^{+}-n-N_{A}^{-}\right)
$$

where $\varepsilon$ is the material permittivity and $\psi$ is the electric potential.

(2) The current-density equations: 


$$
\begin{aligned}
& \vec{J}_{n}=q \mu_{n} n \vec{E}+q D_{n} \nabla n \\
& \vec{J}_{p}=q \mu_{p} p \vec{E}-q D_{p} \nabla p
\end{aligned}
$$

where $\mu_{n}\left(\mu_{p}\right)$ is the mobility of electrons (holes), $\vec{E}=-\nabla \psi$ is the electric field, and $D_{n}=\left(k_{B} T / q\right) \mu_{n}\left(D_{p}=\left(k_{B} T / q\right) \mu_{p}\right)$ is the diffusion constant of electrons (holes). In Equations (2.10), it is assumed that the drift velocity is equal to $\vec{v}_{d}=\mu_{n} \vec{E}$ or $\mu_{p} \vec{E}$.

At sufficiently high filed, $\mu_{n} \vec{E}$ or $\mu_{p} \vec{E}$ should be replaced by the saturation velocity $\vec{v}_{s}$.

(3) The continuity equations:

$$
\begin{aligned}
& \frac{\partial n}{\partial t}=G_{n}-R_{n}+\frac{1}{q} \nabla \cdot \vec{J}_{n} \\
& \frac{\partial p}{\partial t}=G_{p}-R_{p}-\frac{1}{q} \nabla \cdot \vec{J}_{p}
\end{aligned}
$$

where $G_{n}\left(G_{p}\right)$ is the generation rate of electrons (holes), and $R_{n}\left(R_{p}\right)$ is the recombination rate of electrons (holes) [6].

\subsection{5 $\mathrm{SiO}_{2}$ and $\mathrm{Si} / \mathrm{SiO}_{2}$ interface}

One reason that silicon became the dominant semiconductor material used in integrated circuits is that its oxide $\mathrm{SiO}_{2}$ is a stable and high-quality insulator and 
is easy to grow. In a stoichiometric $\mathrm{SiO}_{2}$, each silicon atom is bonded to four oxygen atoms and each oxygen atom is bonded to two silicon atoms as shown in Figure 2.8. Thermally grown $\mathrm{SiO}_{2}$ is an amorphous silicon dioxide structure (shown in Figure.2.9) which is a continuous random network of the basic structure shown in Figure 2.8 [11].

The interface between thermally grown $\mathrm{SiO}_{2}$ and the bulk silicon is a complicated structure, and has been studied extensively because the quality of $\mathrm{Si} / \mathrm{SiO}_{2}$ interface affects the performance of MOSFET (Metal-Oxide-Semiconductor Field-Effect-Transistor) devices. It was proposed that at a $\mathrm{Si} / \mathrm{SiO}_{2}$ interface, between the single-crystal $\mathrm{Si}$ and the stoichiometric, strain-free, amorphous $\mathrm{SO}_{2}$,

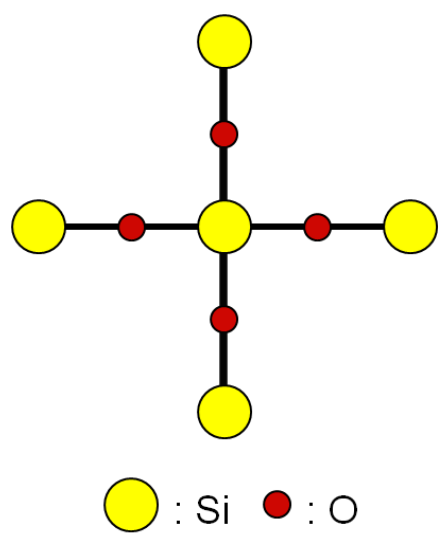

Figure 2.8: Schematic bonding structure in $\mathrm{SiO}_{2}$. ( In crystalline $\mathrm{SiO}_{2}$, the bond angle is $109.5^{\circ}$ ). 


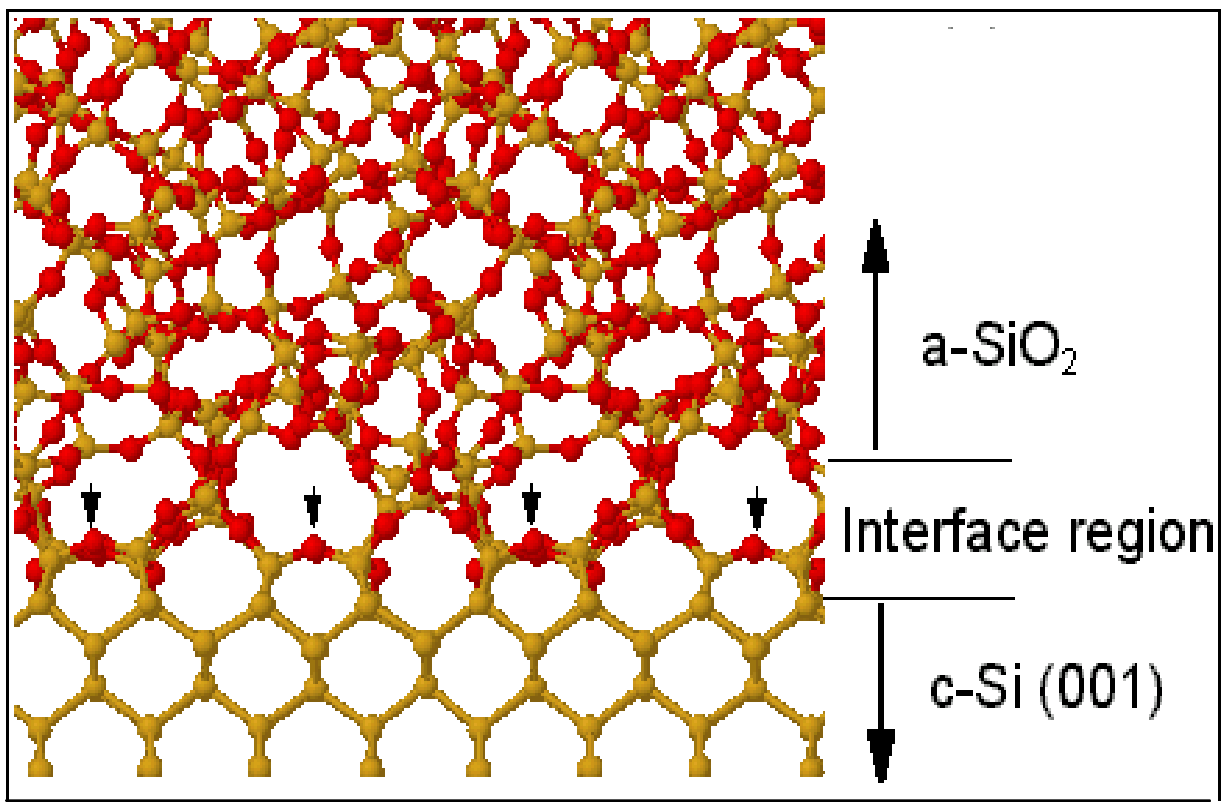

Figure 2.9: Amorphous $\mathrm{SiO}_{2}$ structure (figure taken from Ref. [11] ).

there is a monolayer of nonstoichiometric $\mathrm{SiO}_{\mathrm{x}}(1<\mathrm{x}<2)$ and then a $10-40 \AA$ strained stoichiometric amorphous $\mathrm{SiO}_{2}$ area as shown schematically in Figure 2.9 [11].

Interface traps and/or oxide charge exist at $\mathrm{Si} / \mathrm{SiO}_{2}$ interfaces and in thermally grown $\mathrm{SiO}_{2}$. These interface traps and oxide charges can be classified as the following ( Figure 2.10) [12]: (1) interface traps and interface trapped charge $Q_{i t}$ which come from the silicon dangling bonds at the interrupted silicon surface. These interface traps may be created by the chemical reaction 


$$
\mathrm{Si}-\mathrm{Si}+(\mathrm{O}) \rightarrow \equiv \mathrm{Si} \cdot+(\mathrm{Si}-\mathrm{O})^{+}+e^{-}
$$

where $\equiv \mathrm{Si} \cdot$ is the dangling bond and the $(\mathrm{Si}-\mathrm{O})^{+}$gives rise to the fixed oxide charge $Q_{f}[13]$. These traps can be donor-like or acceptor-like and can exchange electron with the bulk silicon very quickly. These interface traps and interface trapped charges may decrease the carrier mobilities, change the surface potential, and can act as recombination/generation centers. Usually interface traps at $\mathrm{Si} / \mathrm{SiO}_{2}$ interfaces have a density of $10^{11}-10^{12} / \mathrm{cm}^{2} / \mathrm{eV}$ and can be reduced to the order of $10^{10} / \mathrm{cm}^{2} / \mathrm{eV}$ by annealing in $\mathrm{H}_{2}$ at $450^{\circ}$ [6]; (2) The fixed oxide charges (represented by $Q_{f}$ ) are located within $30 \AA$ of the $\mathrm{Si} / \mathrm{SiO}_{2}$ interface and are not mobile [14]. They cannot be charged or discharged. The density is around $10^{10}$ $10^{12} / \mathrm{cm}^{2}$ and depends on the silicon crystal orientation: $\operatorname{Si}(111)>\operatorname{Si}(110)>$ $\operatorname{Si}(100)$ [15]; (3) oxide trapped charges (represented by $Q_{o t}$ ) may be created by irradiation of X-rays, $\gamma$ rays and hot-electron injection; (4) mobile ionic charges $Q_{m}$. $Q_{m}$ are mainly due to ions such as $\mathrm{Na}^{+}, \mathrm{K}^{+}$and $\mathrm{H}^{+}$, which are introduced unintentionally during the processing steps. For example, $\mathrm{Na}^{+}$can come from chemical-cleaning solutions, cleaning glassware, and the oxidation furnace wall. Since these charges are mobile and can redistribute under an applied field, they affect the flat-band potential $[6,16]$ in a time-dependent way. 


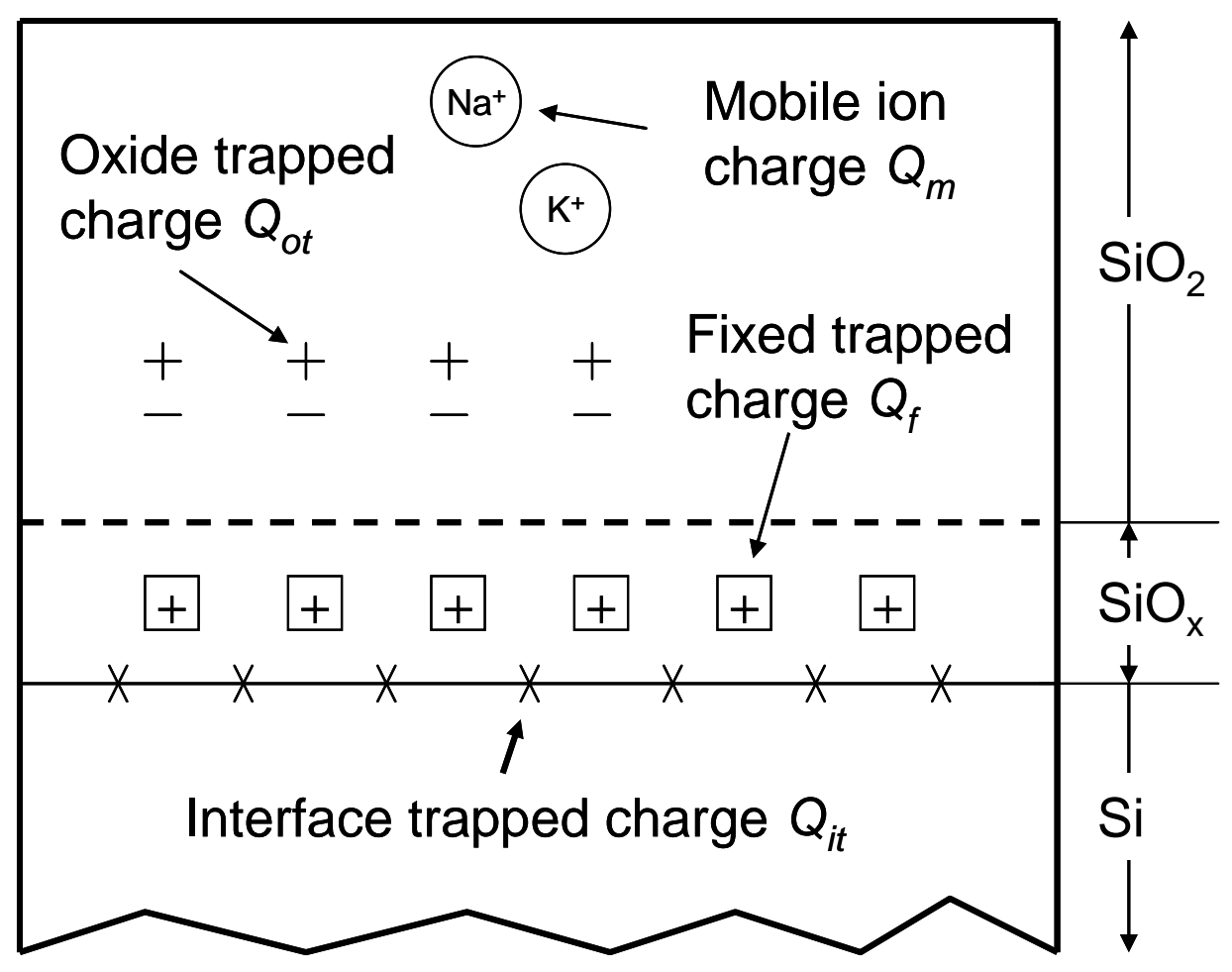

Figure 2.10: Terminology of charges associated with thermal $\mathrm{SiO}_{2}$ [12].

\subsection{Silicon-on-Insulator (SOI)}

Silicon-on-insulator is a three-layer wafer structure as shown in Figure 2.11. For CMOS (complementary metal-oxide semiconductor) based devices fabricated on a bulk silicon, all the devices are connected by the bulk substrate. This can cause some problems such as parasitic capacitance between the source and drain and the 


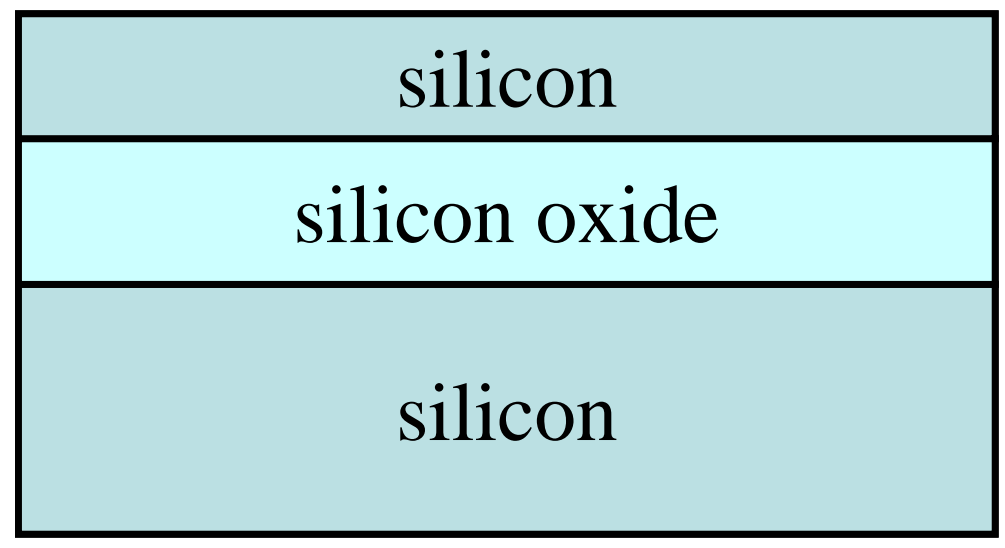

Figure 2.11: Silicon-on-insulator wafer structure.

substrate, and the interaction between the devices [17]. Silicon-on-insulator (SOI) technology is able to avoid these problems by isolating the n-well structures, the pwell structures, and the substrate by an insulating silicon oxide. Compared to bulk devices, SOI devices also have the advantages such as higher speed, lower power dissipation, high radiation tolerance, etc. [17]. SOI technology is also compatible with most conventional fabrication processes.

Several methods of fabricating SOI wafers have been developed, such as SIMOX (Separation by IMplantation of Oxygen), wafer boding and epitaxial growth. Since the SOI wafers used in our experiment is SIMOX SOI, we will only talk about SIMOX in this section.

SIMOX technology includes two steps which are shown in Figure 2.12 [18]. The first stage is implanting a large dose of reactive $\mathrm{O}^{+}$ions into single 


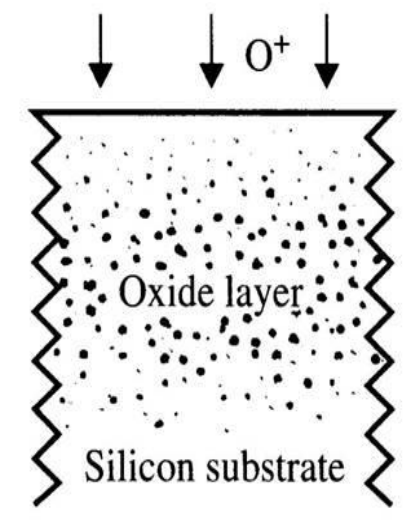

Stage 1

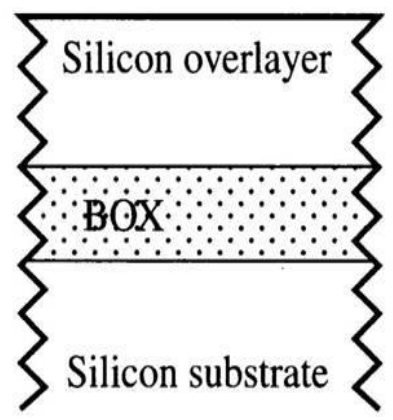

Stage 2

Figure 2.12: The basic SIMOX process (figure taken from Ref. [18]).

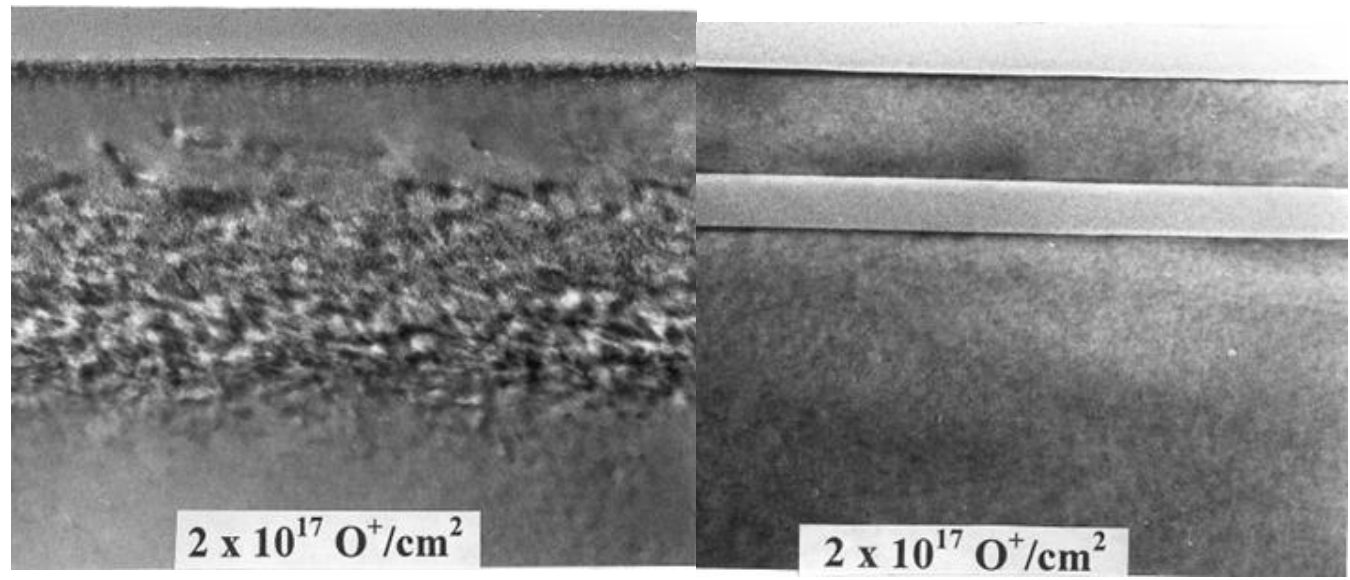

Figure 2.13: Cross-section TEM images of SIMOX samples before and after high temperature annealing at $1360{ }^{\circ} \mathrm{C}$ for 6hours (figure taken from Ref. [19]). 


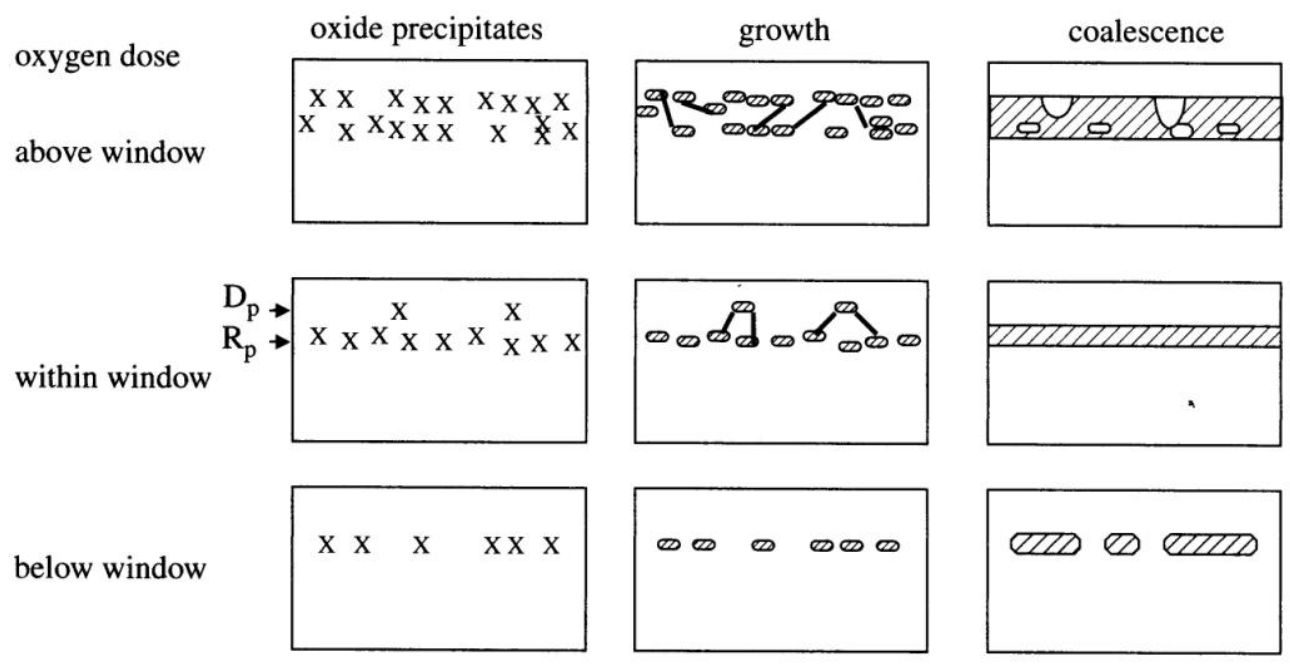

Figure 2.14: Oxygen precipitation, growth and precipitate coalescence during SIMOX annealing with different oxygen doses (figure taken from Ref. [18]).

crystal silicon. The second stage is a high temperature annealing (HTA) step which is aimed at annihilating the lattice defects created during the implantation step and redistributing the implanted oxygen to form a buried layer of homogeneous, stoichiometric $\mathrm{SiO}_{2}$ with abrupt $\mathrm{Si} / \mathrm{SiO}_{2}$ interfaces by the thermally driven physical processes of precipitate growth and precipitate coalescence. Figure 2.13 shows two cross-section transmission electron microscope (TEM) images of SIMOX samples implanted at $65 \mathrm{KeV}$ with a dose of $2.0 \times 10^{17} \mathrm{O}^{+} / \mathrm{cm}^{2}$ before and after HTA at 1360 ${ }^{\circ} \mathrm{C}$ for 4 hours in $\operatorname{Ar}\left(\mathrm{O}_{2}\right)$ without any protective cap on the surface [19]. What makes SIMOX wafers commercially available is two major breakthroughs in 
SIMOX technology: the discovery of low-dose SIMOX [19] and the discovery that internal thermal oxidation [20] occurs during the HTA step.

It was found that for certain implantation energy there is an optimum implantation dose (Izumi window) for the formation of a continuous buried oxide (BOX) [19]. The mechanism is illustrated in Figure 2.14 [18]. When the oxygen dose is above this optimum dose window, too many nucleation sites exist over a wide range. This will result in formation of silicon islands within the BOX film. When the oxygen dose is below this optimum dose window, individual $\mathrm{SiO}_{2}$ islands will form instead of a continuous BOX film. Only when the oxygen dose is within this window, will a continuous BOX form without any silicon island trapped inside [18]. Within the Izumi dose window, the concentration of implanted oxygen atoms is lower than that in stoichiometric $\mathrm{SiO}_{2}$. After the low-dose oxygen implantation, the wafers are annealed in an oxygen rich atmosphere $\mathrm{Ar}+\mathrm{x}_{2}(10 \%<\mathrm{x}<70 \%)$ at high temperature (over $1300{ }^{\circ} \mathrm{C}$ ), which will lead to internal thermal oxidation. Under these conditions, oxygen will be driven by the concentration gradient from the free surface to the BOX and internal thermal oxidation will occur there and predominantly at the SOI/BOX interface [21]. Figure 2.15 shows a set of TEM micrographs of samples implanted at a dose of 1.5, 2.0, 2.5, 3.5, 4.5, and 5.0 $\times 10^{17}$ $\mathrm{O}^{+} / \mathrm{cm} 2$, respectively, but all annealed at $1350^{\circ} \mathrm{C}$ for $6 \mathrm{~h}$ in $\operatorname{Ar}\left(\mathrm{O}_{2}\right)$ with a protective cap [21]. We can see the existence of an optimum dose for the formation of $\mathrm{Si}$ island free BOX layer. 


\section{Annealed with a TEOS cap on the sample surface}
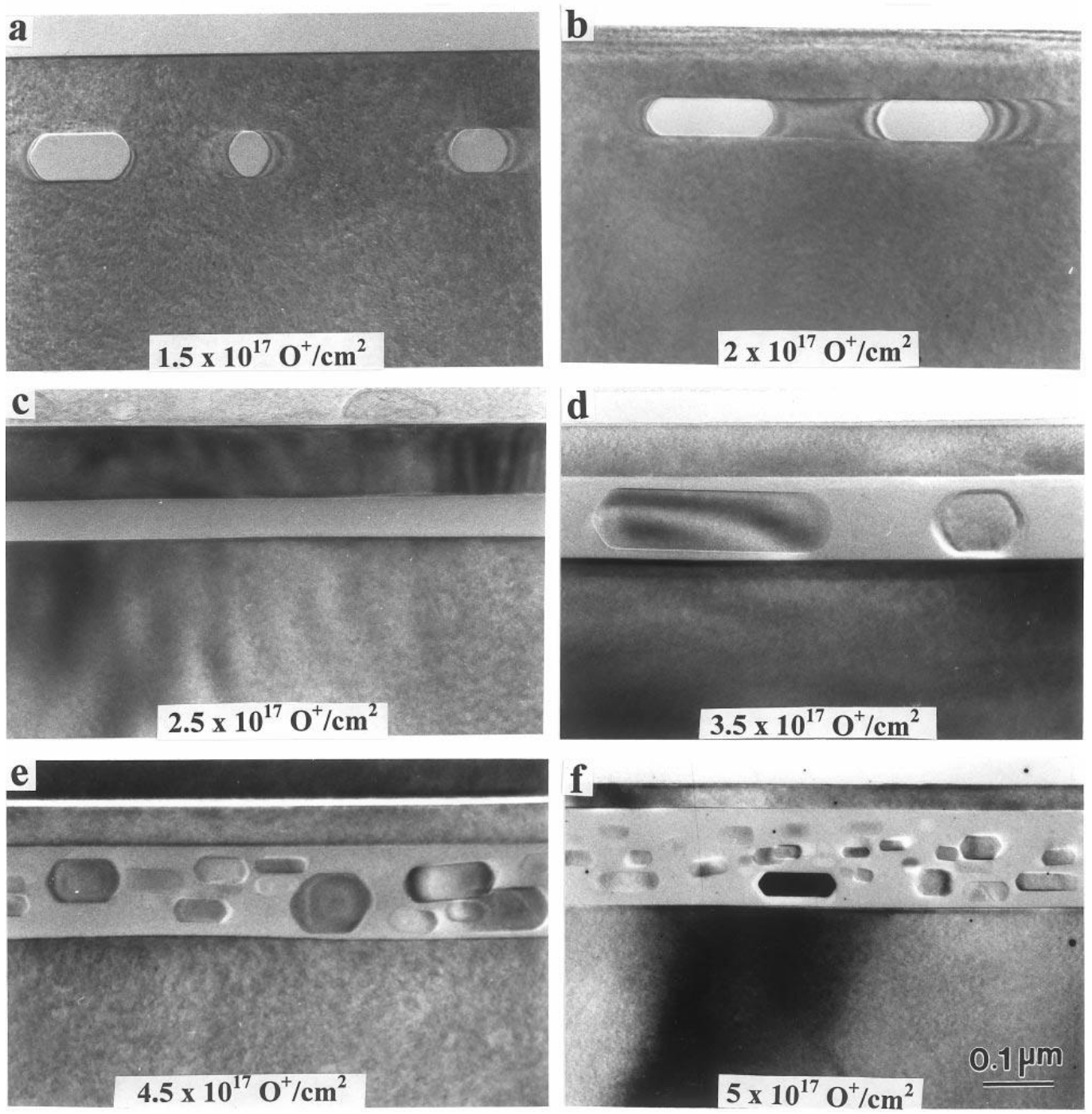

Figure 2.15: A set of TEM micrographs shows the existence of an optimum dose for the formation of Si island free BOX layer. Images (a)-(f) represent samples implanted at a dose of $1.5,2.0,2.5,3.5,4.5$, and $5.0 \times 10^{17} \mathrm{O}^{+} / \mathrm{cm} 2$, respectively but all annealed at $1350^{\circ} \mathrm{C}$ for $6 \mathrm{~h}$ in $\mathrm{Ar}\left(\mathrm{O}_{2}\right)$ with a protective cap (figure taken from Ref. [21]). 
The quality of SOI wafers can be evaluated by the following parameters: SOI thickness and thickness uniformity, BOX thickness and uniformity, HF etch defect density, dislocation density, surface roughness, carrier mobilities, BOX short density, fixed charge density in the BOX, and interface state density at the Si/BOX interfaces. Table 2.1 summarizes typical physical and electrical properties of commercial SIMOX compared with those of bonded SOI [18].

\begin{tabular}{|c|c|c|c|}
\hline Parameters & Unit & SIMOX SOI & Bonded SOI \\
\hline SOI thickness uniformity & $\AA$ & $6-9$ & $10-15$ \\
\hline HF defects & $\mathrm{cm}^{-2}$ & $<0.5$ & $<0.5$ \\
\hline Surface roughness & $\AA$ & $4-7$ & $1.0-2.0$ \\
\hline Electron mobility & $\mathrm{cm}^{-2} / \mathrm{V} / \mathrm{s}$ & $550-650$ & $500-600$ \\
\hline Hole mobility & $\mathrm{cm}^{-2} / \mathrm{V} / \mathrm{s}$ & $200-230$ & $200-230$ \\
\hline BOX short density & $\mathrm{cm}^{-2}$ & $<0.5$ & $<0.5$ \\
\hline BOX breakdown field & $\mathrm{MV} / \mathrm{cm}$ & $7-8$ & $>8$ \\
\hline BOX charge & $\mathrm{cm}^{-2}$ & $3.0 \times 10^{11}$ & $<3.0 \times 10^{11}$ \\
\hline Interface charge & $\mathrm{cm}^{-2}$ & $3.0 \times 10^{11}$ & $<3.0 \times 10^{11}$ \\
\hline
\end{tabular}

Table 2.1: Physical and electrical properties of modern commercial SIMOX [18]. 


\section{CHAPTER 3}

\section{METAL-SEMICONDUCTOR INTERFACE}

\subsection{Schottky Barriers}

When a metal makes abrupt contact with a semiconductor, often the metal Fermi energy falls within the bandgap of the semiconductor. In this case there are energy offsets between the Fermi energy and the conduction band and valence band of the semiconductor. For an n-type semiconductor, the Fermi energy/conduction band offset represents an energy barrier (called an electron Schottky barrier) that majority electrons must overcome to move between the metal and semiconductor. For a p-type semiconductor, the Fermi Energy/valence band offset represents a hole Schottky barrier for majority holes. For an ideal metal/semiconductor interface (i.e., with no interface states), the formation of an electron or hole Schottky barrier can be understood using simple energy-band diagrams. Consider for example an ideal metal/n-semiconductor contact. When the metal and the n-semiconductor are 

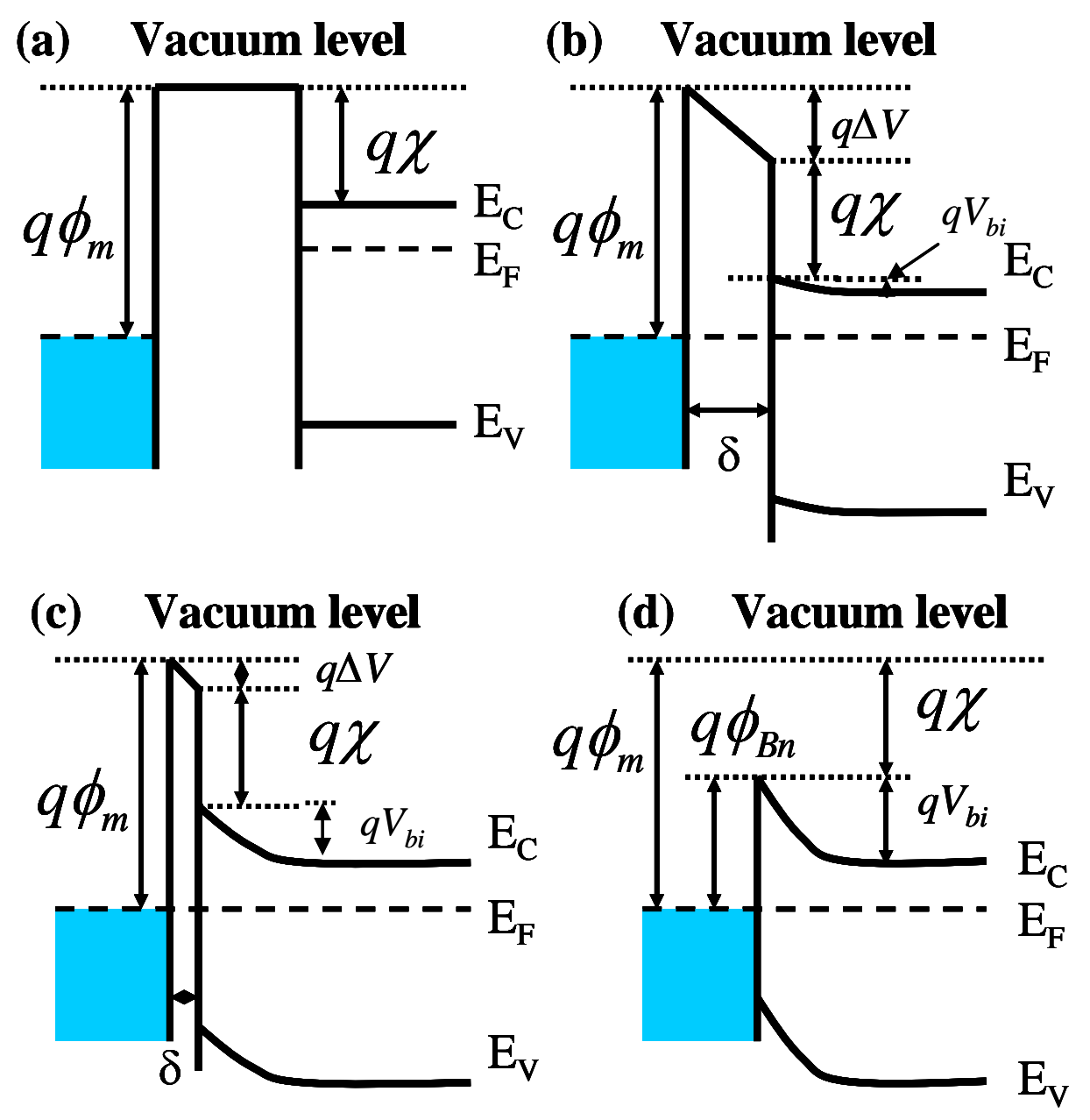

(d) Vacuum level

Figure 3.1: The energy band diagram of metal-semiconductor contacts.

isolated, the vacuum levels of the metal and semiconductor are equal causing the energy band alignment as shown in Figure 3.1(a). If charge transfer is permitted, then electron transfer between the metal and semiconductor cause their Fermi 
energies to line up at thermal equilibrium. Figures 3.1(b) and (c) show the case when the distance between the metal and the semiconductor is finite. We can see the contact potential due to the difference between the metal work function $q \phi_{m}$ and the semiconductor work function $q \chi+\left(E_{C}-E_{F}\right)$ (where $q \chi$ is the semiconductor electron affinity) is shared by the voltage drop $\Delta V$ across the space between the metal and the semiconductor and the band bending $V_{b i}$ in the semiconductor. The voltage drop $\Delta V$ decreases as the spacing decreases and eventually goes to zero when the metal makes intimate contact with the semiconductor as show in Fig. 3.1(d). Based on the above arguments, the barrier height can be easily obtained from the band diagram:

$$
q \phi_{B n}=q \phi_{m}-q \chi
$$

This is called Schottky-Mott model.

\subsection{Fermi Level Pinning}

According to the Schottky-Mott model, the change of the barrier height should scale exactly with the change of metal work function, i.e., $q \Delta \phi_{B n}=q \Delta \phi_{m}$ for different metal contacts to the same semiconductor. However experimental measurements showed that for many metal/semiconductor contacts (including those made to $\mathrm{Si}$ and GaAs) the dependence of the barrier height on the metal work 
function is weaker: $q \Delta \phi_{B n}=\alpha * q \Delta \phi_{m}$, where $\alpha<<1$ ( e.g. $\alpha=0.27$ for Si and $\alpha=$ 0.074 for GaAs - see Figure 3.2) [22].

To explain this, Bardeen proposed that between the metal and the semiconductor there exists an interfacial layer which is of atomic dimensions $(\delta \sim$

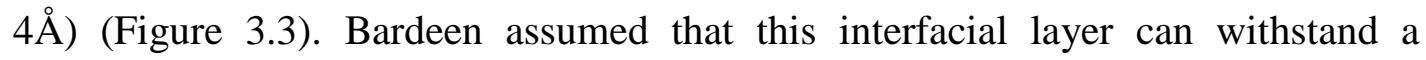
potential drop $\Delta V$ across it but is transparent to electrons. In this interfacial layer,

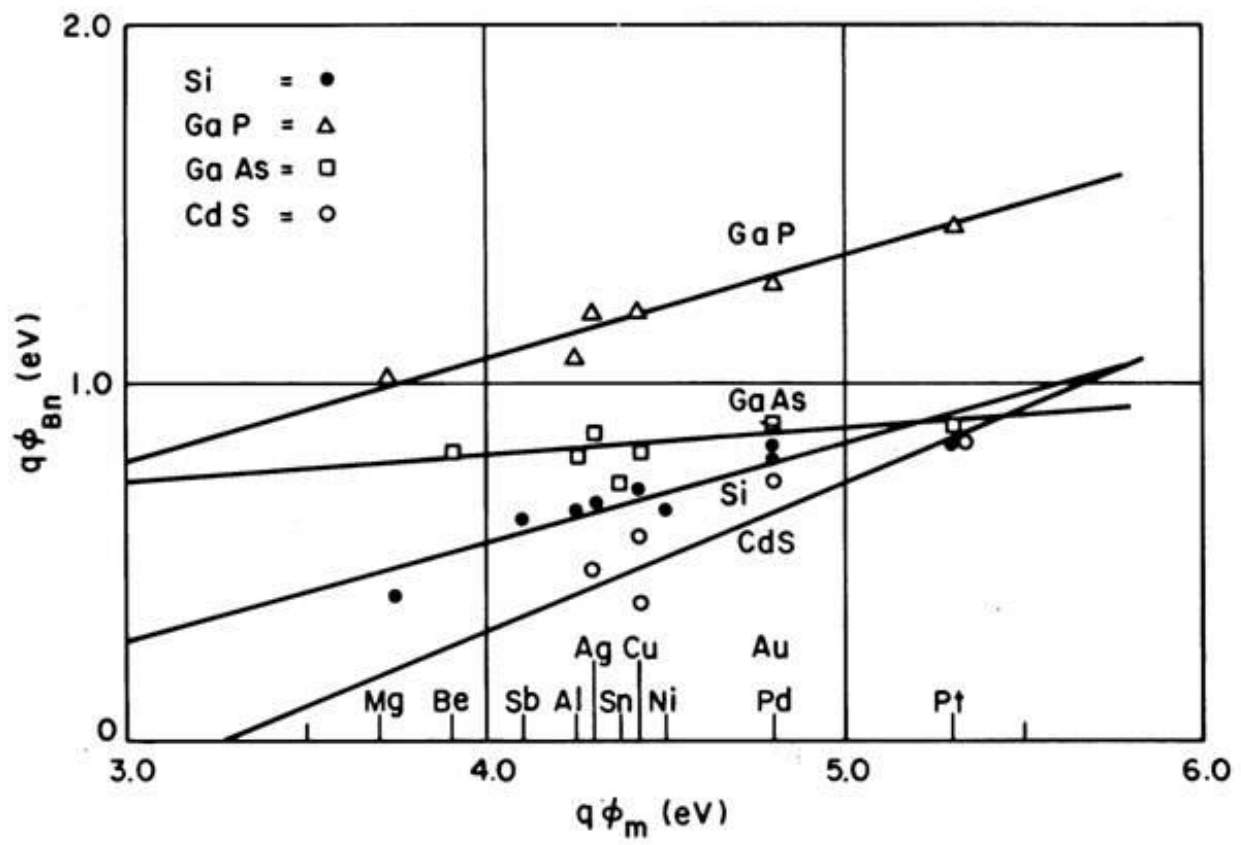

Figure 3.2: Experimental results of barrier height for metal-semiconductor contacts (figure taken from Ref. [22]). 
Bardeen proposed that there is a large density of interface states which can become charged according to

$$
\sigma_{s}=q D_{s}\left(q \phi_{C N L}-E_{F}\right),
$$

where $D_{s}$ is the interface-state density in unit of $1 / \mathrm{cm}^{2} / \mathrm{eV}$ and $q \phi_{C N L}$ is called the charge neutrality energy level. When $E_{F}=q \phi_{C N L}$, there is no surface charge; when

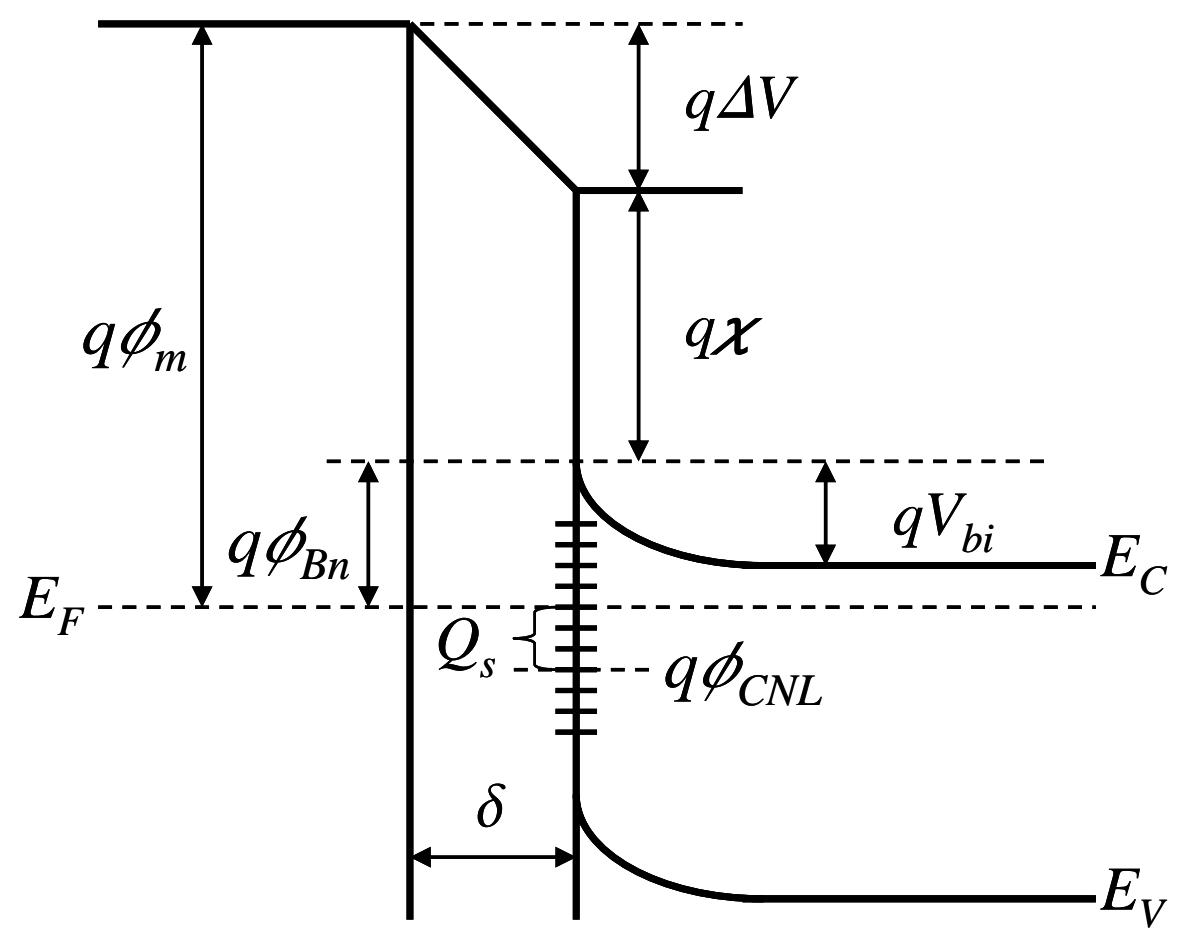

Figure 3.3: Energy band diagram with interface states [23]. 
$E_{F}$ is above $q \phi_{C N L}$, interface charge is negative, and when $E_{F}$ is below $q \phi_{C N L}$, interface charge is positive. This interface charge and its image charge inside the metal form a planar charge- dipole sheet. If the contact size is $>>\delta$ (which is always true for large planar contact to bulk semiconductors), the size of this dipole sheet can be considered as infinite, so the potential drop across the interfacial layer due to this infinite dipole sheet is equal to $\Delta V=\sigma_{s} \delta / \varepsilon_{0}=q D_{s}\left(q \phi_{C N L}-E_{F}\right) \delta / \varepsilon_{0}$. If $D_{s}$ is very large, then only a small deviation of $E_{F}$ from $q \phi_{C N L}$ would create a large density of interface charge. The net effect is that a change in the metal work function causes a change in the interface charge that produces a potential drop $\Delta V$ across the interfacial layer which can accommodate the change of the metal work function. In this case, the Fermi level is completely pinned at the charge neutrality level $q \phi_{C N L}$, so the barrier height doesn't depend on the metal work function and is equal to

$$
q \phi_{B n}=E_{C}-q \phi_{C N L}
$$

When $D_{s}$ is equal to zero, the Schottky barrier is determined by Equation (3.1). For real semiconductors such as $\mathrm{Si}$ and $\mathrm{GaAs}, D_{s}$ is large but finite, causing partial pinning with:

$$
q \phi_{B n}=a\left(q \phi_{m}-q \chi\right)+b\left(E_{C}-q \phi_{C N L}\right)
$$


where $a=2 q \varepsilon_{s} N_{D} \delta^{2} / \varepsilon_{i}^{2} a=$ and $b=1-\varepsilon_{s} /\left(\varepsilon_{i}+q^{2} \delta D_{s}\right)$ are two constants determined by the semiconductor and the interface states $\left(\varepsilon_{i}\right.$ is the permittivity of the interface layer, and $\varepsilon_{s}$ is the permittivity of the semiconductor, and $N_{D}$ is the doping of the semiconductor) [6]. Actually several models have been proposed to explain the physical origin of these interface states, (for example surface states [6], MIGS (Metal-Induce-Gap-States) [24] and impurity states [25]), but none of them can explain all of the experimental results. However, their basic effect on the Schottky barrier is the same: interface states, an interfacial layer, and a charge neutrality level $q \phi_{C N L}$ provide a "negative feedback" for the movement of Fermi level responding to the change of the metal work function.

\subsection{Image Force Lowering}

Since the metal in a metal-semiconductor contact has a very high free carrier density, the effective electrical potential $V(x)$ that an electron inside the semiconductor feels is modified so that there is an "image force" induced lowering of the Schottky barrier height that depends on the electric field near the metalsemiconductor interface. Let's use a metal-vacuum system shown in Figure 3.4 as an example to explain the basic idea of image-force-induced lowering. When an 


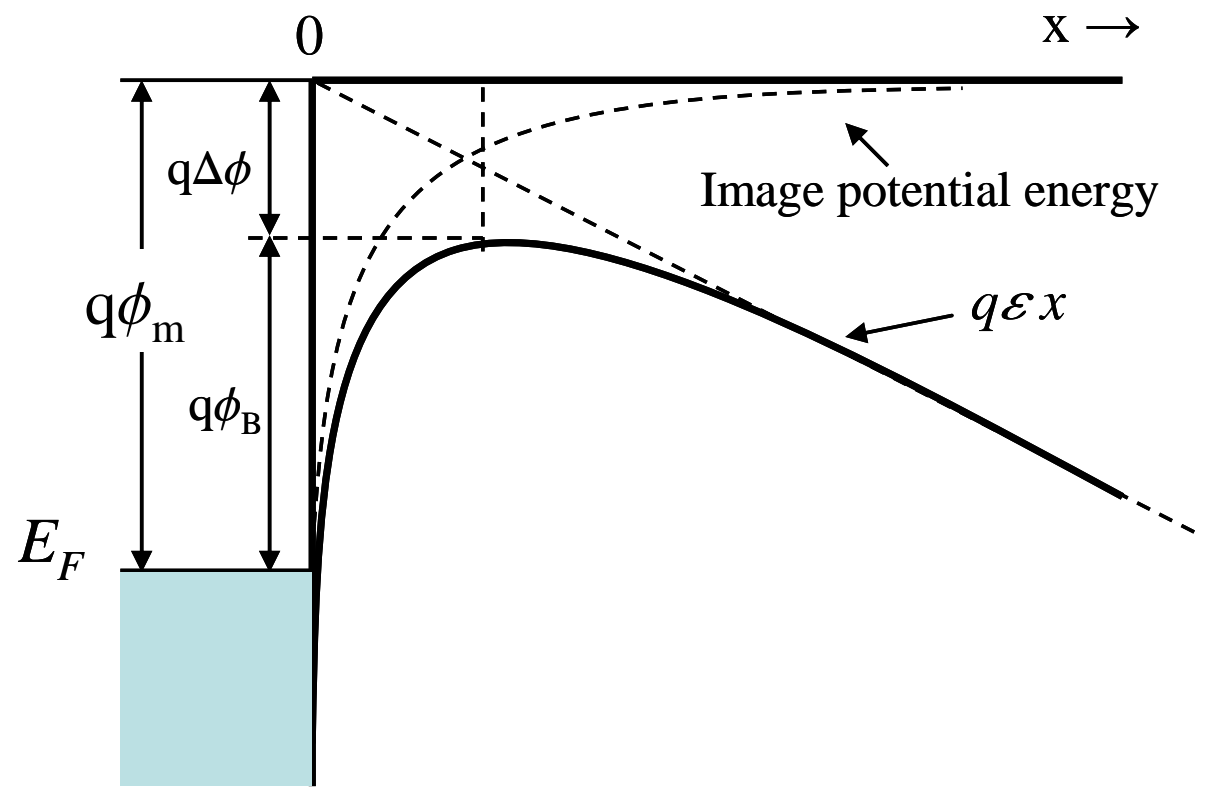

Figure 3.4: Energy band diagram between a metal and a vacuum [6].

electron is placed at a distance $x$ from the metal, the force between the electron and the metal is attractive and is easy to be calculated using the image charge method [26]. For an infinite flat metal, the force is equal to that generated by an equal positive charge $q^{+}$at position $-x$, so the image force is

$$
F=\frac{-q^{2}}{16 \pi \varepsilon_{0} x^{2}},
$$


where $\varepsilon_{0}=8.85 \times 10^{-12} \mathrm{~F} / \mathrm{m}$ is the vacuum permittivity. And the potential due to this image force is

$$
V(x)=\frac{q}{16 \pi \varepsilon_{0} x} .
$$

If an external field $E$ exists at the interface, the total potential energy is

$$
q V(x)=-\frac{q^{2}}{16 \pi \varepsilon_{0} x}-q E x .
$$

The maximum of $q V(x)$ can be found by the condition $d V(x) / d x=0$. And the image force lowering of the Schottky barrier can be found to be

$$
q \Delta \phi_{I F L}=q \sqrt{\frac{q E}{4 \pi \varepsilon_{0}}} .
$$

For a semiconductor, $\varepsilon_{0}$ should be replaced the semiconductor dielectric constant, and experimental results showed that for metal-silicon contacts the $\varepsilon_{0}$ should be replaced by the static dielectric constant of silicon $\varepsilon_{\mathrm{s}}=11.7 \varepsilon_{0}$ [27]. 


\subsection{Current Transport}

The current transport through a thick Schottky barrier can be described by the thermionic emission theory which assumes that only carriers with a kinetic energy greater than the energy barrier can pass over the barrier, and that there is no transport through the barrier via tunneling or generation-recombination processes. Using the density of states and the Fermi distribution function, the current density from metal to semiconductor $J_{m-s}$ and the current density from semiconductor to metal $J_{s-m}$ can be calculated. The net current density is $J_{T E}=J_{m-s}+J_{s-m}$ and can be calculated to be

$$
J_{T E}=A^{*} T^{2} \exp \left(-\frac{q \phi_{B}}{k_{B} T}\right)\left[\exp \left(\frac{q V}{k_{B} T}\right)-1\right]
$$

where $A^{*}$ is the effective Richardson constant and $V$ is the applied voltage [28]. Figure 3.5 shows the theoretical and experimental values of current-voltage characteristics for $\mathrm{Au} / \mathrm{Si}$ barrier [29].

In the thermionic emission theory, the current component due to the tunneling through the barrier is neglected, which is not a valid approximation for heavily doped semiconductors and low temperature operation. For heavily doped semiconductors, the Schottky barrier can be so thin that carriers can tunnel through the barrier. At low temperature, the number of carriers which have a higher kinetic 


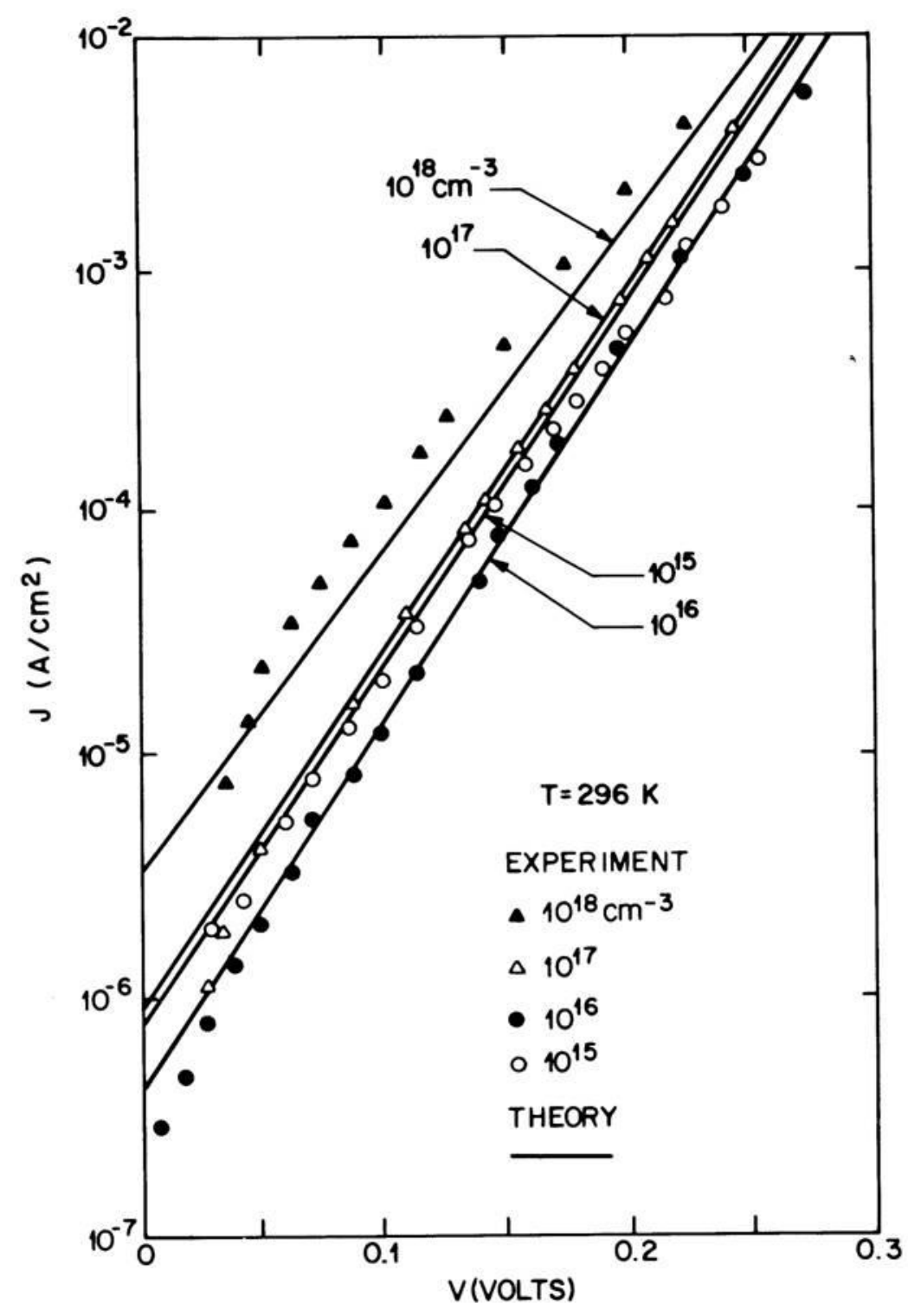

Figure 3.5: Theoretical and experimental values of current-voltage characteristics for $\mathrm{Au} / \mathrm{Si}$ barrier (Figure taken from Ref. [29]). 
energy than the energy barrier will become too small. So in these cases the tunneling current density may dominate and it can be written as

$$
J_{t} \propto \exp \left(-2 \phi_{B} / \hbar / \sqrt{N_{D}\left(\operatorname{or} N_{A}\right) /\left(\varepsilon_{s} m^{*}\right)}\right)
$$

where $\hbar$ is the reduced Plank constant and $m^{*}$ is the tunneling effective mass of the carriers [30].

For a real device, the current density can be written as

$$
J=J_{S}\left[\exp \left(\frac{q V}{n k_{B} T}\right)-1\right]
$$

where $J_{S}$ is the saturation current density, and $n$ is the ideality factor which is equal to 1 for an ideal Schottky diode and is between 1 and 2 for a real Schotty diode because of the reasons such as image force lowering, tunneling, surface imperfection, recombination in depletion region, etc. [6]. 


\subsection{Ohmic contacts}

In semiconductor devices, ohmic contacts are usually needed and are defined as contacts which have a negligible contact resistance and give a linear and symmetric $I-V$ curve. Figure 3.6 (a) and (b) show two ways which are used to fabricate ohmic contacts: low barrier and/or high doping [6]. In the case of low barrier height, due to thermal excitation, the number of carriers which can transport over the barrier by the thermionic emission mechanism is very large, so the resistance is small. In the case of high doping, the density of ionized dopants is high and the bands bend steeply. This gives rise to a very thin barrier so the carriers can easily tunnel through it. In practice, it may be difficult to fabricate a metal/semiconductor contact with a low barrier height, so high doping is the most frequently used method for making an ohmic contact to semiconductors.

\subsection{The Tung Model: inhomogeneous Schottky Barriers}

In the above discussion, a uniform Schottky barrier height was assumed at the metal/semiconductor interface. However, small-scale lateral variations of the Schottky barrier height should produce different current-voltage behavior than predicted for a uniform barrier. Such local variations in Schottky barrier height have been observed in several studies using ballistic electron emission microscopy (BEEM) [31-34]. For example, Niedermann et al. saw a lateral variation of more than $0.2 \mathrm{eV}$ in barrier height in their BEEM studies of $\mathrm{Pt} / \mathrm{n}-\mathrm{Si}(100)$ [31]. Palm et al. 


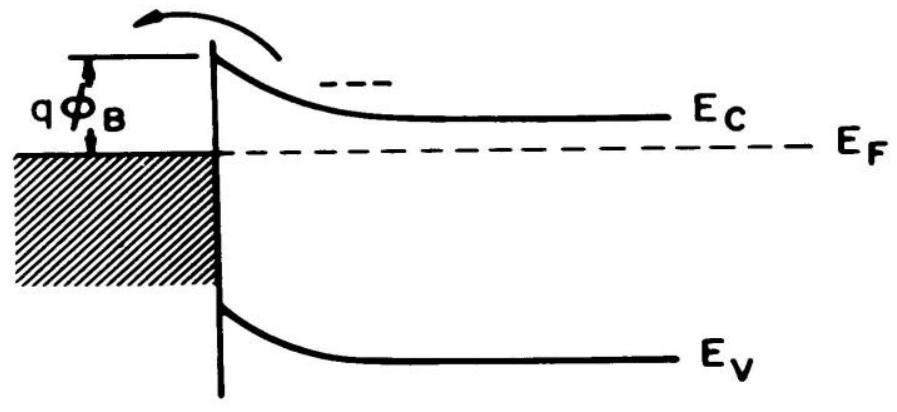

(a) LOW BARRIER HEIGHT

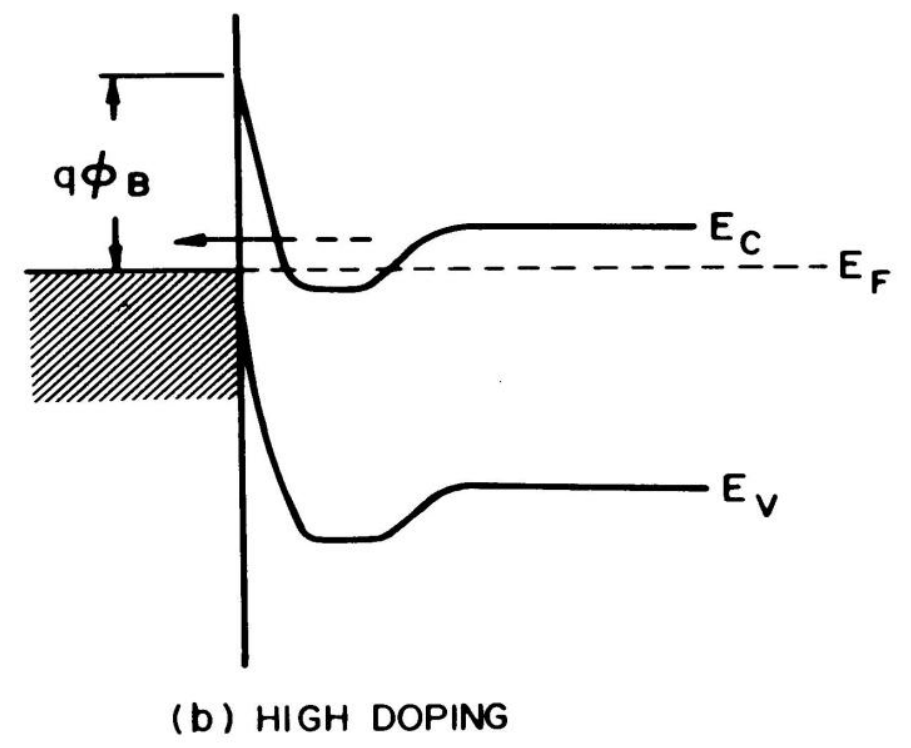

Figure 3.6: Low barrier height and/or high doping for ohmic contacts (Figure taken from Ref. [6]). 
studied the distribution of barrier heights of $\mathrm{Au} / \mathrm{n}-\mathrm{Si}(100)$ contacts for two different dopant concentration and found that the width of the distribution is larger for the sample with higher doping [32]. One possible reason for this barrier height inhomogeneity is the presence of semiconducting or insulation interlayers at the interface due to interfacial reactions or contaminations.

A general theoretical treatment of the barrier height inhomogeneity has been done by Tung $[35,36]$. In Tung's model, the metal/semiconductor interface consists of patches each of which has a different local Schottky barrier height, and the conduction band minimum potential inside the semiconductor is obtained by solving Poisson's equation with the boundary condition determined by the barrier heights at the interface. Simulation results showed that due to the interaction between neighboring patches with different barrier heights the conduction path beneath a small patch with a low barrier height is "pinched-off' by the presence of high barrier height patches in its close proximity. This is illustrated in Fig. 3.7, which plots the calculated conduction band minimum potential in an n-type semiconductor near a circular patch based on Tung's model [31]. The radius of the circle is $10 \mathrm{~nm}$ and has a barrier height of $0.4 \mathrm{eV}$. The area outside the circle has a barrier height of $0.6 \mathrm{eV}$ and a depletion width of $1 \mathrm{um}$. We can see beneath the lower barrier patch the conduction band minimum increases to a saddle point about $25 \mathrm{~nm}$ away from the interface inside the semiconductor. When pinch-off occurs, the potential at a saddle point beneath the low barrier height area determines the transport properties [35]. The effect of such a saddle point on electrical transport 


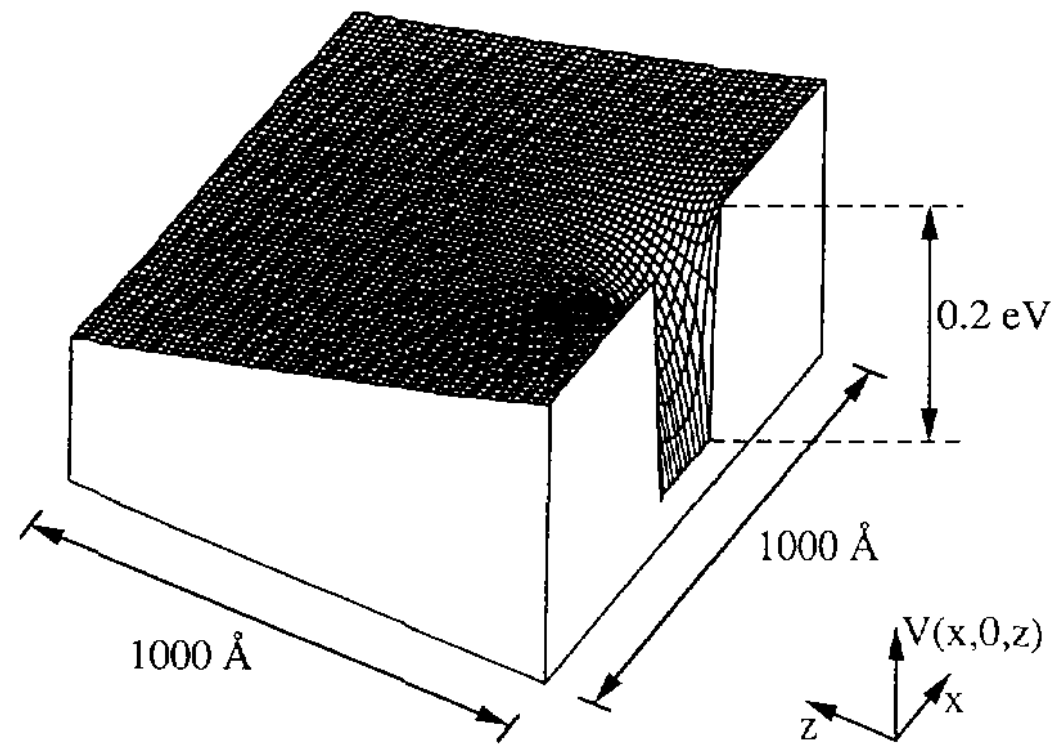

Figure 3.7. Conduction band minimum potential in an n-type semiconductor near a circular patch of radius $=10 \mathrm{~nm}$ with a $0.2 \mathrm{eV}$ lower barrier height. The outside barrier height is $0.6 \mathrm{eV}$ and the depletion width is $1 \mathrm{um}$. (figure taken from Ref. [31]).

across the Schottky barrier should become significant if the size of a low-barrier patch is smaller than the characteristic depletion width of the semiconductor $[35,36]$. 


\subsection{Characterization of Schottky Barrier Height}

The experimental method for measuring the Schottky barrier height includes current-voltage measurement, activation energy measurement, capacitance-voltage measurement, photoelectric measurement and BEEM (Ballistic Electron Emission Microscopy). The method of BEEM will be discussed in the next chapter, and a summary of the other five methods mentioned above can be found in reference [6]. 


\section{CHAPTER 4}

\section{BALLISTIC ELECTRON EMISSION MICROSCOPY}

\subsection{Schematics of BEEM}

The technique of Ballistic Electron Emission Microscopy (BEEM) was invented by W.J.Kaiser and L.D.Bell in 1988 to investigate metal/semiconductor interfaces with high spatial $(\sim$ few $\mathrm{nm})$ and energy $(\sim 10 \mathrm{meV})$ resolution [37]. This is a three-terminal technique modified from Scanning Tunneling Microscopy (STM) and has been mainly used to measure Schottky barrier heights and hot carrier transportation at metal/semiconductor interfaces. BEEM has also been applied to study energy band offsets [38], quantum-size effects [39] using semiconductor heterostructures, and spin-dependent electron scattering and spin-filtering effects [40] using magnetic metal/semiconductor interfaces. BEEM has also been applied to study electron transport in insulator using metal/insulator/semiconductor structures 
(a)

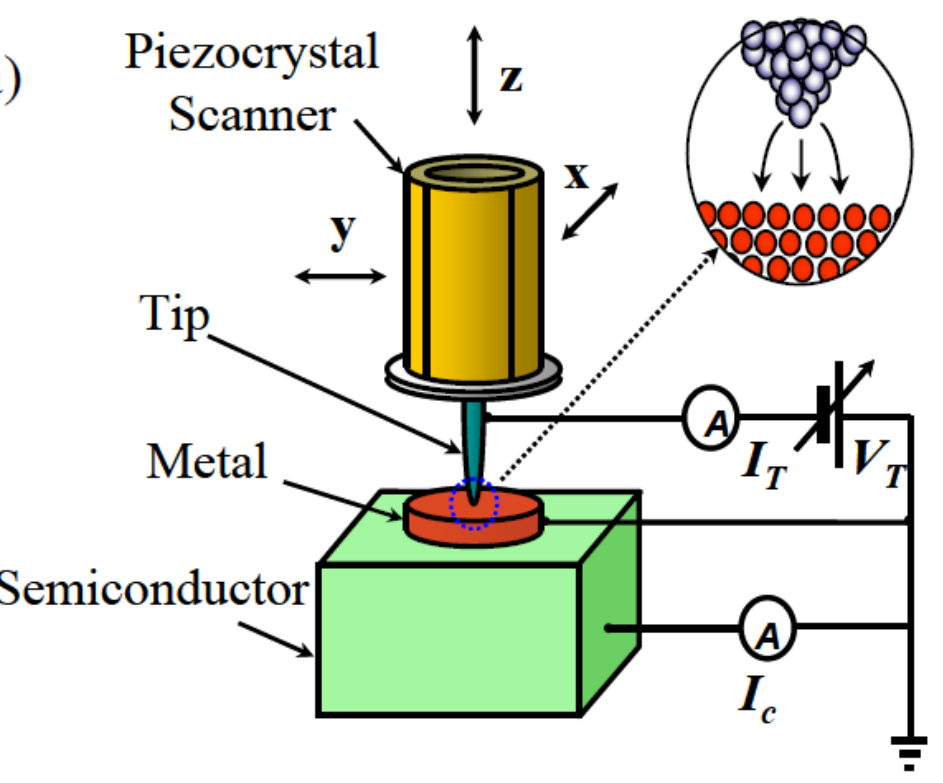

(b) $\stackrel{\mathrm{z}}{\longrightarrow}$ Schottky contact

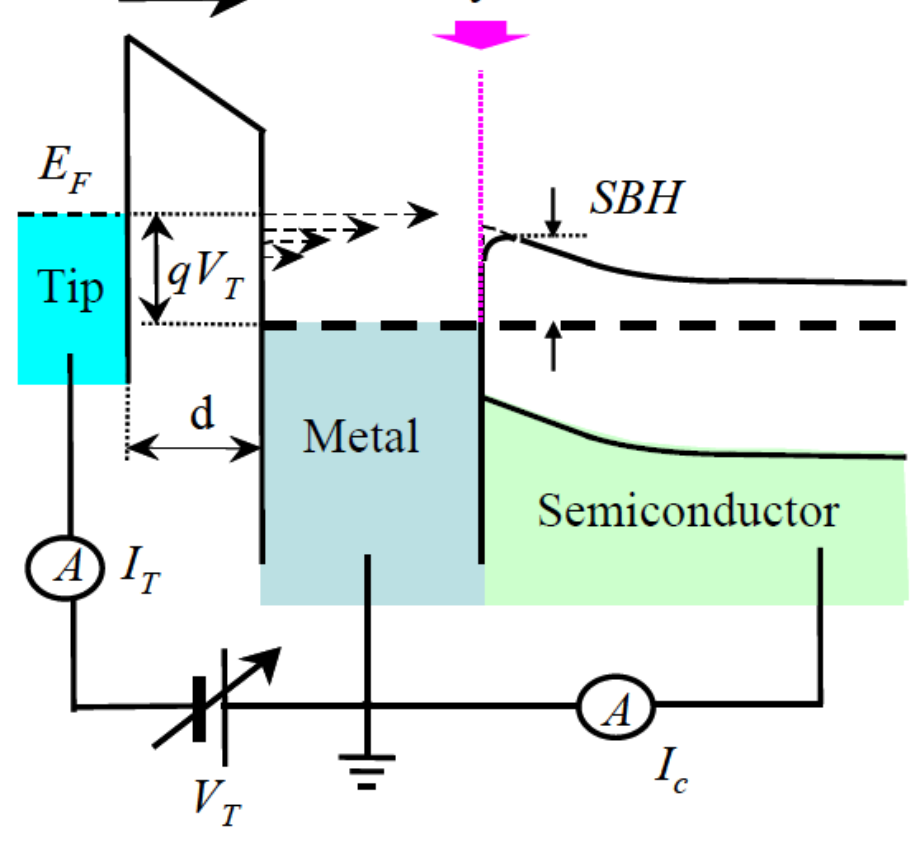

Figure 4.1: (a) Schematic representation and (b) Energy band diagram of BEEM . (Figure taken from Ref. [46]). 
[41-43]. Reference [44] and [45] are two good comprehensive reviews of BEEM technique.

The basic experimental setup of BEEM measurements is illustrated in Figure 4.1(a) [46]. The energy band diagram of BEEM measurements is shown in Figure 4.1(b) using a metal/n-semiconductor interface as an example [46]. In typical BEEM measurements, small size (diameter $\sim 500 \mathrm{um}$ ) Schottky contacts are fabricated by evaporating a thin $(\sim 10 \mathrm{~nm})$ and clean metal film on a clean semiconductor surface (usually with native oxide removed). With the metal film grounded, if a negative bias $V_{T}$ is applied on the STM tip and the tip is brought close $(\sim 1 \mathrm{~nm})$ to the metal film, electrons can tunnel through the barrier between the tip and the metal film and enter the metal films as hot electrons with an average energy about $q V_{T}$ higher than the metal Fermi energy. Because the metal film is very thin, a small portion of these hot electrons can survive thermalization and reach the metal/semiconductor interface without losing significant energy. There the Schottky barrier works like an energy filter: only electrons with energy higher than the Schottky barrier height can cross the interface and contribute to the BEEM current $I_{c}$. (Acually only a fraction of the electrons with enough energy can cross due to mechanisms such as defect, scattering or because lateral momentum is not conserved). The barrier height at the metal/semiconductor interface can be determined as $q V_{t h}$ where $q V_{t h}$ is the threshold tip voltage above which the BEEM current will start to increase from zero. 


\subsection{BEEM Spectrum}

The most important application of the BEEM technique is the determination of the Schottky barrier height at a metal/semiconductor interface by taking BEEM spectra measurements in which the BEEM current $I_{c}$ is measured as a function of the tip voltage $V_{T}$. Figure 4.2 shows a spectrum measured on an Au/n-Si Schottky contact. The black arrow indicates the fitted Schottky barrier height using the method discussed in the next section. Due to the statistical distribution of the

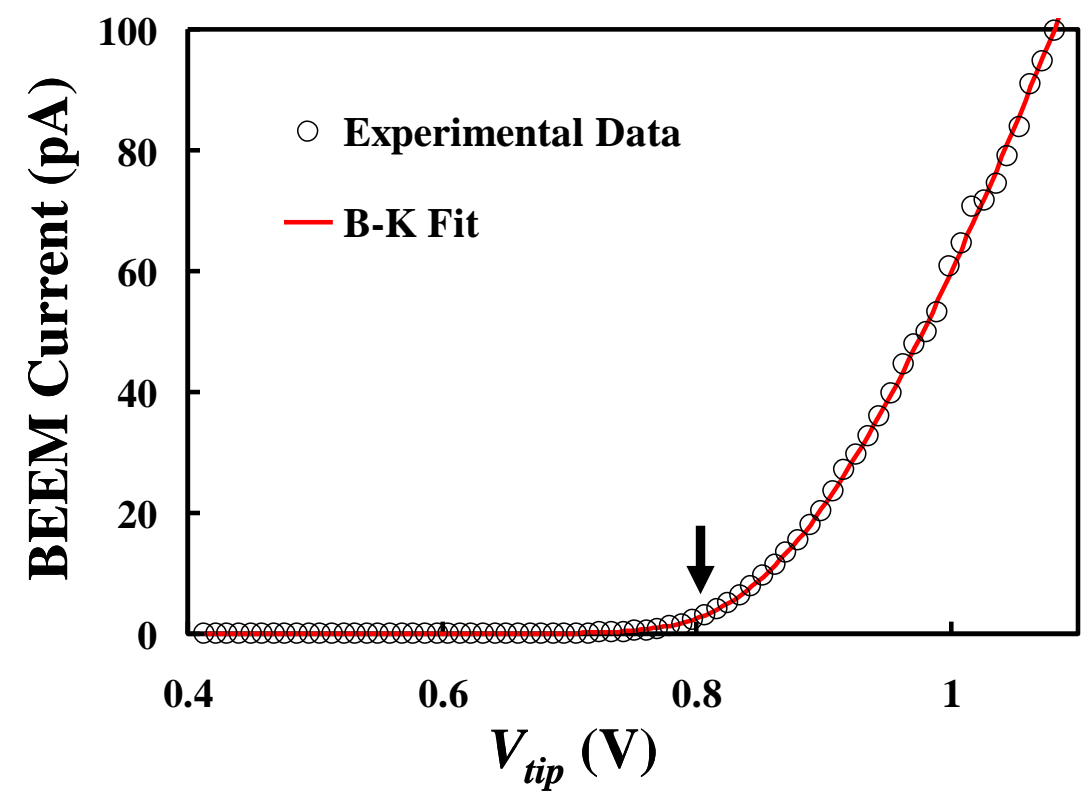

Figure 4.2: Typical BEEM spectrum on $\mathrm{Au} / \mathrm{n}-\mathrm{Si}$. 
injected hot electrons according to the temperature and the Fermi energy inside the tip, the BEEM current actually starts to increase from zero at a voltage which is smaller than the indicated threshold voltage.

\subsection{Theoretical Models}

In order to extract a Schottky barrier height from BEEM measurements, several theoretical models have to be proposed to describe the spectral shape of the BEEM current around the threshold voltage region. Among them, the Bell-Kaiser (BK) model [37, 47] and the Ludeke-Prietsch (LP) model [48,49] are the most commonly used models. In these two models, a thermally broadened power-law ( $n=$ 2 for the BK model and $n=5 / 2$ for the LP model) is assumed to describe the BEEM current at the threshold voltage region.

The transport of electrons in the BEEM process can be divided into four independent steps: (1) tunneling from the tip to the metal film; (2) transport of the hot electrons through the metal film; (3) transmission across the interface from the metal into the semiconductor; (4) transport inside the semiconductor. The general expression of the BEEM current can be written as

$$
I_{B E E M}\left(V_{T}\right)=\int_{-\infty}^{\infty} d E \iint_{2 \pi} d \Omega D\left(E, V_{T}, \vec{u}, T\right) \exp [-l(\vec{u}) / \lambda(E)] C(E, \vec{u})
$$


In Equation (4.1), $D\left(E, V_{T}, \boldsymbol{u}, T\right)$ describes the distribution of tunneling current from the tip to the metal film where $\mathbf{u}$ is the tunneling direction; $\exp [-l(\boldsymbol{u}) / \lambda(E)]$ describes the transmission through the metal film where $l(\boldsymbol{u})$ is the electron-path length through the metal film and $\lambda(E)$ is the energy-dependent mean-free path of the electrons; and $C(E, \boldsymbol{u})$ describes the transmission coefficient across the metalsemiconductor interface. In the following parts of this section, we will discuss how to calculate BEEM current using Equation (4.1) in the BK model and the LP model.

\section{(1) Tunneling current distribution.}

Here a planar tip approximation has been assumed to describe the tunneling between the tip and the metal film [50]. The distribution of the tunneling current can be written as

$$
D\left(E, V_{T}, \vec{u}, T\right) \propto \rho_{T}\left(E-q V_{T}\right) \rho_{M}(E)\left[f\left(E-q V_{T}\right)-f(E, T)\right]|\mathrm{M}|^{2}
$$

In Equation (4.2), $\rho_{T}\left(E-q V_{T}\right)$ and $\rho_{M}(E)$ are the density of states in the tip and the metal film, $f\left(E-q V_{T}, T\right)$ and $f(E, T)$ are the Fermi distribution functions in the tip and the metal film respectively, where the Fermi distribution is defined as $f(x, T)=1 /\left(1+\exp \left(x / k_{B} T\right)\right)$, and $|\mathrm{M}|^{2}$ is the tunneling probability (Figure 4.3). For

a square tunneling barrier, using the Wentzel-Kramers-Brillouin (WKB) approximation, the tunneling probability can be estimated as 


$$
|M|^{2} \cong \exp \left(-\frac{2 \sqrt{2 m}}{\hbar} \beta d \sqrt{W_{A}+q V_{T} / 2-\left(E-E_{/ /}\right)}\right),
$$

where $m$ is the free electron mass, $\beta \sim 1$ is a correction factor, $d$ is the vacuum gap, $W_{A}=\left(W_{T}+W_{M}\right) / 2$ is the average of the work functions of the tip and the metal film, $E_{/ /}$is the incident electron's kinetic energy parallel to the metal surface, and $W_{A}+$ $q V_{T} / 2-\left(E-E_{/ /}\right)$is the average potential barrier height.

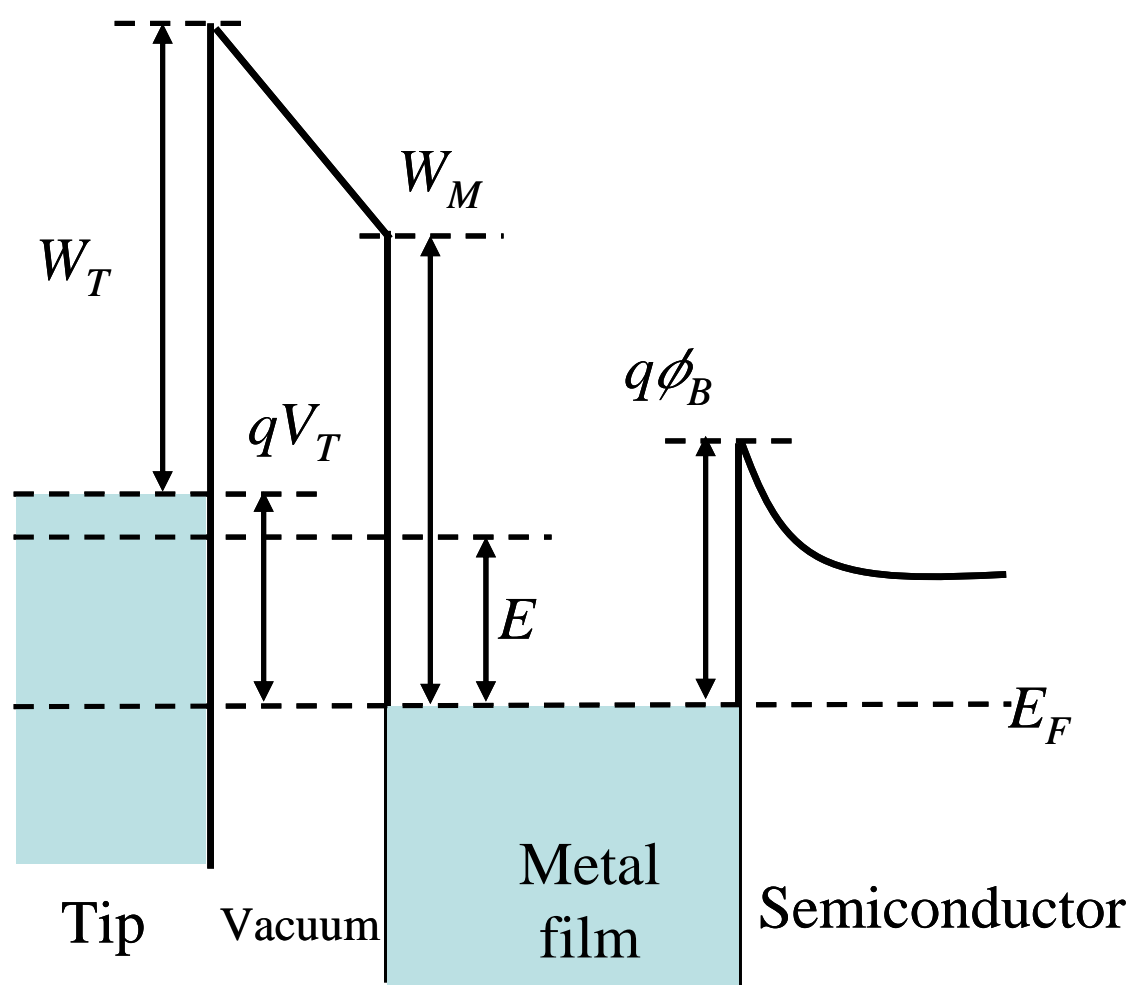

Figure 4.3: Schematic energy diagram of the tunneling process. 
Compared to the strong energy variation of the tunneling probability defined as Equation (4.3), the densities of states $\rho_{T}\left(E-q V_{T}\right)$ and $\rho_{M}(E)$ can be assumed to be constant in the energy region of BEEM measurements, so the spatial and energy distribution of the tunneling current is mainly determined by Equation (4.3). And it can be shown that the energetic and spatial distribution of the tunneling current is approximately described by

$$
D\left(E, V_{T}, \vec{u}, T\right) \propto f\left(E-q V_{T}\right) \exp (E / \Delta E) \exp \left(\left(-\varphi_{T} / \Delta \varphi_{T}\right)^{2} / 2\right)
$$

where $\Delta E \cong 0.2 \mathrm{eV}, \varphi_{T}$ is the tunneling angle relative to the normal direction of the metal film, and $\Delta \varphi_{T} \cong 20^{\circ}$ [44]. $\Delta E \cong 0.2 \mathrm{eV}$ means the tunneling probability increase by $\mathrm{e}=2.718$ times when the energy is increased by $0.2 \mathrm{eV} . \Delta \varphi_{T} \cong 20^{\circ}$ describes the angular distribution of the tunneling probability, which means most of the tunneling happens within an angle $\leq 20^{\circ}$ relative to the surface normal. This average opening angle of the tip-emission cone is derived from a sharp tip and is larger than the value estimated for planar tunneling $\left(\Delta \varphi_{T} \cong 11^{\circ}\right.$ for Au film), since in reality a laterally infinite tip is not a good approximation.

\section{(2) Transport through the metal film.}

During the transport through the metal film, the hot electrons will suffer from inelastic scattering and elastic scattering: inelastic scattering will result in a background of secondary electrons, and elastic scattering will result in a larger 
effective path length and a more isotropic angular distribution [44]. The main contribution to the inelastic scattering is coming from the electron-electron scattering which gives rise to a mean-free path of $\lambda(E) \cong\left(E-E_{0}\right) / E^{2}$ where $E_{0}$ is the conduction band minimum in the metal film. Both of inelastic scattering and elastic scattering will broaden the momentum distribution of the hot electrons and reduce the lateral spatial resolution of BEEM [44].

In the BK model and the LP model, the mean-free path of the electrons in the metal film $\lambda(E)$ is assumed to be energy- independent, and the factor $\exp [-l(\boldsymbol{u}) / \lambda(E)]$ in Equation (4.1) is replaced by unity.

\section{(3) Transmission across the interface.}

In the following discussion about the transmission of hot electrons across the metal/semiconductor interface, two assumptions have been made: (1) free-electron like behavior in both the metal and the semiconductor and (2) conservation of both energy and transverse momentum of electrons. So just before and after the electrons cross the interface,

$$
E=\frac{\hbar^{2} k_{\perp, M}^{2}}{2 m}+\frac{\hbar^{2} k_{/, M}^{2}}{2 m}-E_{F}=q \phi_{B}+\frac{\hbar^{2} k_{\perp, S}^{2}}{2 m_{\perp}}+\frac{\hbar^{2} k_{/ /, S}{ }^{2}}{2 m_{/ /}}
$$

and

$$
k_{/ /}=k_{/ /, M}=k_{/ /, S},
$$


where $m$ is the electron mass in the metal film, $m_{\perp}$ and $m_{/ /}$are the longitudinal and transverse effective mass of electrons in the semiconductor, and the superscript $M$ and $S$ mean the values in the metal and in the semiconductor respectively (Figure 4.4). It will only be possible for an electron with an energy $E$ to cross the interface if the Equations (4.5) and (4.6) have real number solutions for $k_{\perp, M(S)}$ and $k_{/ /, M(S)}$. It can be shown using Figure 4.4 that only when the incident angle $\theta_{M}$ inside the metal film satisfies

$$
\sin ^{2} \theta_{M} \leq \frac{m_{/ /}}{m} \frac{E-q \phi_{B}}{E+E_{F}},
$$

an electron with an energy $E$ can cross the interface. Equation (4.7) defines an acceptance cone with an opening angle

$$
\theta_{C, M}=\arcsin \sqrt{\frac{m_{\|}}{m} \frac{E-q \phi_{B}}{E+E_{F}}}
$$

If transverse momentum is indeed conserved, then all the electrons with angles larger than $\theta_{C, M}$ should be reflected back to the metal film at the interface and can't enter the semiconductor. For $\mathrm{Au} / \mathrm{Si}$ contacts if we assume $m_{/ /} \cong m, \theta_{C, M}$ is equal to $7^{\circ}$ for $E-q \phi_{B}=0.1 \mathrm{eV}$ and $16^{\circ}$ for $E-q \phi_{B}=0.5 \mathrm{eV}[44]$. 


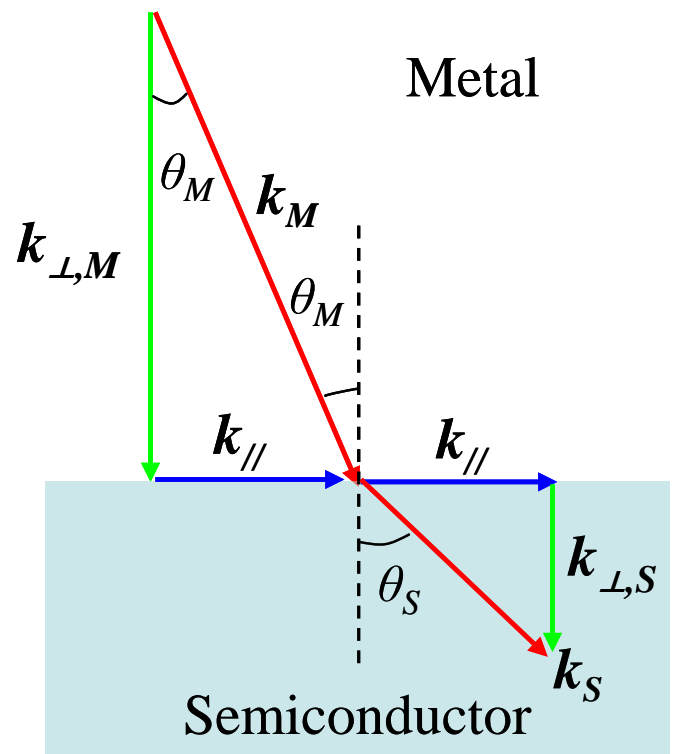

Figure 4.4: $k$-space diagram of electrons crossing the interface.

In the BK model, it is assumed that for all the electrons which satisfy the condition (4.7) the transmission coefficient across the metal-semiconductor interface $C(E, \boldsymbol{u})$ is constant. But in the LP model, the Schottky barrier is assumed to be an abrupt potential step, and the transmission coefficient $C(E, \boldsymbol{u})$ can be calculated using quantum mechanics to be

$$
C(E, \vec{u})=\frac{4 k_{\perp, M} k_{\perp, S}}{\left(k_{\perp, M}+k_{\perp, S}\right)^{2}} .
$$


Based on the discussion in (1)-(3), the total BEEM current in the threshold region is approximately given by

$$
I_{B E E M}\left(V_{T}\right) \propto \int_{q \phi_{B}}^{\infty} d E \frac{-d f\left(E-q V_{T}, T\right)}{d E}\left(E-q \phi_{B}\right)^{n}
$$

where $n=2$ for the BK model and $n=5 / 2$ for the LP model. This equation represents a thermally broadened $n$-power law. The difference $1 / 2$ in the power $n$ comes from the difference in the transmission coefficient $C(E, \boldsymbol{u})$.

Since in our experiment we use the BK model to fit our results, here we will say a little more about the BK model. The discussion below follows the reference [51] with the symbols modified to be consistent with the discussion above. For the case of $m_{/ /}<m$, using Equations (4.5) and (4.6), it can be shown that electrons which can cross the Schottky barrier and enter the semiconductor need satisfy the following two equations:

$$
E_{/ /} \leq \frac{m_{/ /}}{m-m_{/ /}}\left(E_{\perp}-E_{F}-q\left(V_{T}+\phi_{B}\right)\right) \equiv E_{/ /}^{\max }
$$

and

$$
E_{\perp} \geq E_{F}+q\left(V_{T}+\phi_{B}\right) \equiv E_{\perp}^{\min }
$$


So the BEEM current can be written as

$$
I_{B E E M}\left(V_{T}\right)=R I_{t 0} \frac{\int_{E_{\perp}^{\min }}^{\infty} d E_{\perp} D\left(E_{\perp}\right) \int_{0}^{E_{/ /}^{\max }} d E_{/ /}\left(f\left(E-q V_{T}, T\right)-f(E, T)\right)}{\int_{0}^{\infty} d E_{\perp} D\left(E_{\perp}\right) \int_{0}^{\infty} d E_{/ /}\left(f\left(E-q V_{T}, T\right)-f(E, T)\right)} .
$$

To extract barrier heights, experimental spectra are fitted by Equation (4.13) with $\phi_{B}$ and $R$ as adjusting parameters. This model in general fits our data in the nearthreshold pretty well as shown in Figure 4.2. And even if it is not exactly correct for our experiments, it is still a well-defined fitting formula that should accurately reveal changes in the Schottky barrier height if they exist.

(4) Transport in the semiconductor.

After electrons enter the semiconductor, several things can happen: (1) some electrons can be reflected back to the metal due to elastic scattering; (2) energydependent back reflection in the region between the interface and the potential maximum due to the inelastic scattering by optical phonons will decrease the overall transmission coefficient [52]; and (3) impact ionization can result in a larger BEEM current than given in Equation (4.10) by generating electron-hole pairs [53-55]. 


\subsection{Equipment Setup}

Our BEEM measurements were performed in an ultra high vacuum (UHV) system with a base pressure of $1-2 \times 10^{-10}$ Torr. This UHV system was modified from a commercially available Omicron variable temperature STM. Details about this setup are available in the Ph.D thesis of Eric Heller [56].

This UHV system is composed of an airlock and two main chambers (an analysis chamber and an STM chamber). The airlock is equipped with a magnetically coupled horizontal transfer arm which allows the transfer of samples in and out of the UHV system without breaking the vacuum of the main chambers. The analysis chamber is connected to the airlock and has one horizontal arm and one vertical arm that allow the sample transfer from the airlock to the STM chambers. The horizontal arm can also allow direct and indirect heating samples up to $~ 1400 \mathrm{~K}$ for direct heating and $\sim 750 \mathrm{~K}$ for indirect heating. A Varian Vac-Ion Plus 500 ion pump with a pumping speed of 500 litters/sec is connected to the analysis chamber in order to maintain a $\sim 1-2 \times 10^{-10}$ Torr pressure inside the system. Inside the analysis chamber, there is also a tip/sample carousel which can hold up to six tips/samples. The STM chamber has one CCD (charge-coupled device) camera which is used to locate the sample and position the tip or the grounding Au wires. The STM chamber also has a liquid helium flow cryostat which is connected to the sample stage with a copper braid. This allows the sample to be cooled down to $\sim 25$ 


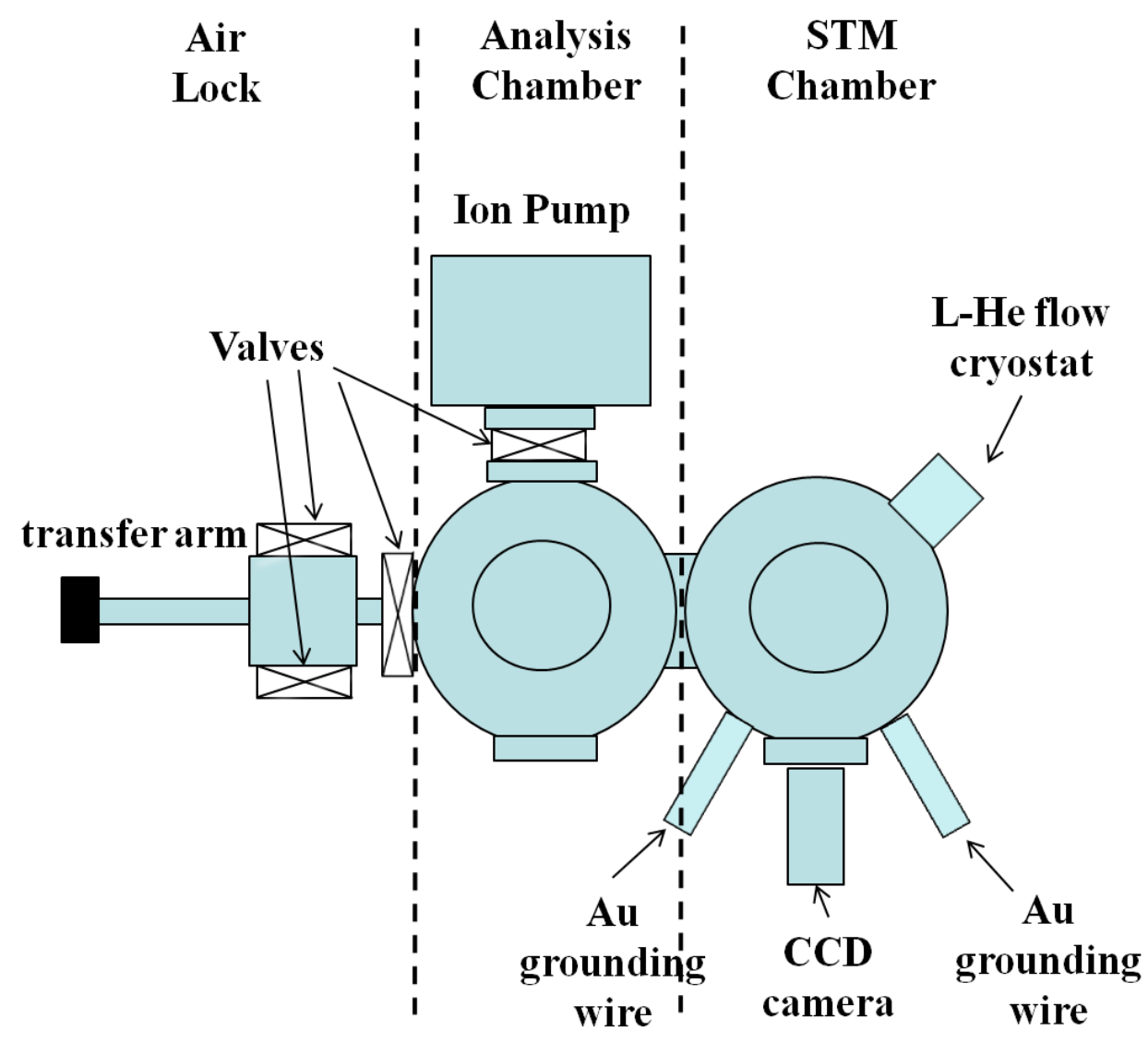

Figure 4.5: Schematic of our Omicron variable temperature STM system.

K. A current preamplifier is built inside the STM chamber so it is very close to the sample stage to reduce electronic noise. A tip voltage up to $10 \mathrm{~V}$ and a tunnel current up to $25 \mathrm{nA}$ can be used to scan areas as large as $100 \mu \mathrm{m}^{2}$. 
Camelia Marginean and I modified the system to add a second grounding $\mathrm{Au}$ wire. This Au wire is not easy to manipulate as the Au wire on the left side since the motion in $\mathrm{x}$ and $\mathrm{y}$ direction is not independent. 


\section{CHAPTER 5}

\section{SAMPLE FABRICATION}

In this chapter, we will discuss our SOI sample structure and the process procedures used in our sample fabrications in details.

\subsection{Sample structure}

In our studies, we want to modify the electric field at a metal/semiconductor interface in a sample geometry so that the top metal surface can be accessed by an STM tip. To do this, we take advantage of the three-layer structure of SOI wafer and fabricated a sample structure as shown in Figure 5.1. The Au electrode on the substrate is used to apply a gate voltage to modify the electric field at a metal/semiconductor interface on the top SOI film.

Our SOI samples were fabricated using SIMOX SOI wafers from Simgui Technology Co., China. These SIMOX SOI wafers have a three-layer structure of 


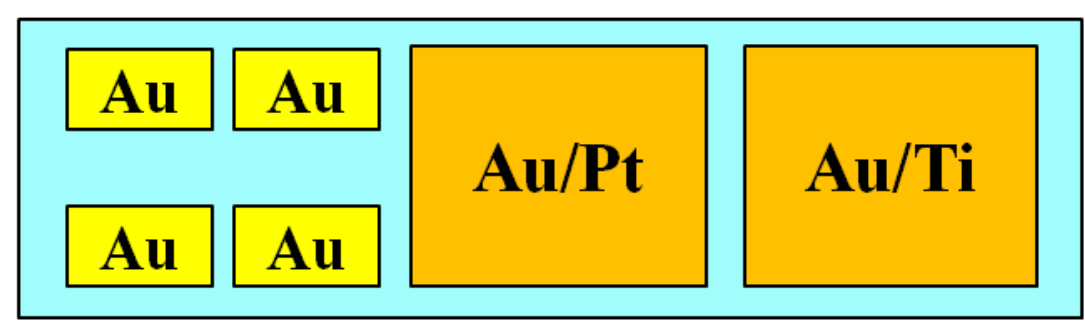

(a)

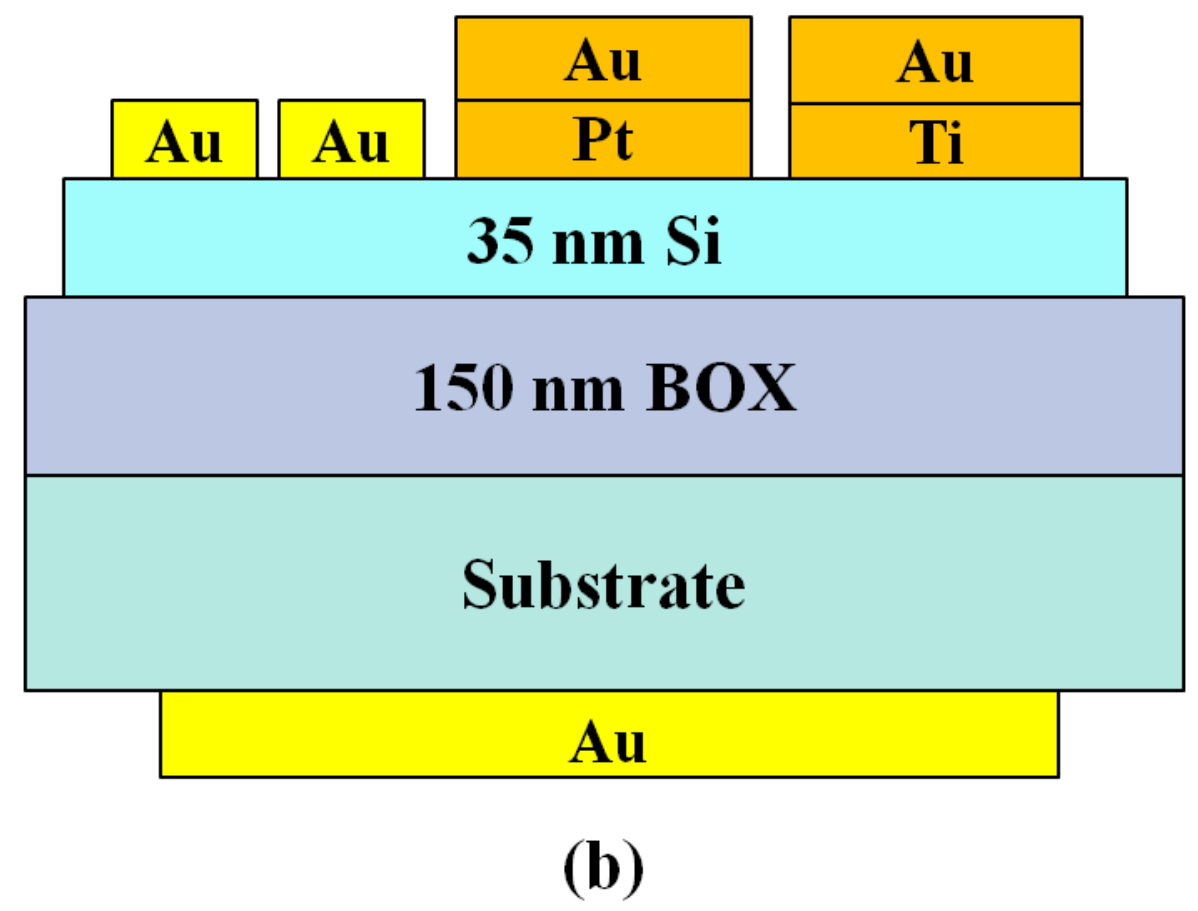

Figure 5.1: Top view and side view of our SOI sample. 
$35 \mathrm{~nm}-\mathrm{Si} / 150 \mathrm{~nm}-\mathrm{SiO}_{2} / \mathrm{p}-\mathrm{Si}$ substrate, where the starting silicon wafer was boron doped at $0.6-1.2 \times 10^{15} / \mathrm{cm}^{3}$. The detail of our SOI sample structure is illustrated in Figure 5.1. To reduce leakage current between the top silicon film and the p-Si substrate, the top silicon film was etched into $\sim 3.5 \mathrm{~mm} \times 0.9 \mathrm{~mm}$ rectangular islands using plasma etching or RIE etching. For measurements on $\mathrm{Au} / \mathrm{Si}$ contacts, four separate $450 \mu \mathrm{m} \times 300 \mu \mathrm{m} \mathrm{Au/Si} \mathrm{Schottky} \mathrm{contacts} \mathrm{were} \mathrm{deposited} \mathrm{on} \mathrm{each}$ silicon island with Au film thickness $=7 \mathrm{~nm}$. The ohmic contact for BEEM measurements is a $950 \mu \mathrm{m} \times 600 \mu \mathrm{m}$ size Ti/Si contact with $20 \mathrm{~nm}$ Ti capped with $100 \mathrm{~nm} \mathrm{Au}$, and the ohmic contact for BHEM measurements is a $950 \mu \mathrm{m} \times 600 \mu \mathrm{m}$ size Pt/Si contact with $20 \mathrm{~nm}$ Pt capped with $100 \mathrm{~nm}$ Au. For measurements on $\mathrm{Cu} / \mathrm{Si}$ contacts, four separate $450 \mu \mathrm{m} \times 300 \mu \mathrm{m} \mathrm{Cu} / \mathrm{Si}$ Schottky contacts were deposited on each silicon island with $20 \mathrm{~nm} \mathrm{Cu}$ capped with $10 \mathrm{~nm} \mathrm{Au}$. The top $\mathrm{Au}$ layer presents to protect the $\mathrm{Cu}$ film from oxidation. A much larger contact $(950 \mu \mathrm{m} \times 600 \mu \mathrm{m})$ of the same composition was found to be a sufficiently leaky "ohmic" contact to collect both electrons and holes from each Si island. A largearea $100 \mathrm{~nm}$ thick Au film electrode was deposited on the backside of the substrate for the back gate bias. Prior to depositing these contacts, the native oxide was removed by dipping in a 10:1 buffered HF (hydrofluoric acid) solution for $20-30$ s. A standard lift-off process was used for each metal contact pad, with metals deposited by thermal or electron beam evaporation. 
At room-temperature, the resistance between each $\mathrm{Si}$ island and the substrate was measured, and samples with one or more islands with $>1 \mathrm{~T} \Omega$ resistance were introduced into our UHV Omicron variable temperature STM/BEEM chamber (base pressure $\sim 1 \times 10^{-10}$ Torr) for BEEM/BHEM measurements [57]. Such a large island/substrate resistance is necessary to reduce stray current from the Si island when a large (up to $17 \mathrm{~V}$ ) substrate back-gate bias is applied.

In our sample fabrications, we need to do wafer cleaning, photolithography, dry etching, metal deposition, lift-off, and wet etching. The details of these process procedures are discussed in the following sections.

\subsection{Chemical cleaning}

Depending on the fabrication jobs and the available facilities, different cleaning methods were used during our sample preparation. In our labs (Smith Laboratory and the Physics Research Building (PRB) at Ohio State University), three-solvent cleaning was used to degrease the wafers. In the cleanroom in the Nanotech West fabrication facility at Ohio State University, either piranha cleaning or four-chemical cleaning was used. 


\subsubsection{Three-solvent cleaning}

In this cleaning method, three solvents Trichloroethalyne (TCE), Acetone (ACE), and Methanol (ME) are used to clean the sample to remove organic contaminations or dusts on the wafer surface. This cleaning process was mainly used to clean silicon or GaAs wafers in our lab for evaporating $\mathrm{Au} / \mathrm{Si}$ or $\mathrm{Au} / \mathrm{GaAs}$ Schottky contacts and we found this is good enough for us. The cleaning procedure is

(1) Place the sample in a clean glass beaker.

(2) Ultra-sonic clean in $\sim 50 \mathrm{ml}$ TCE for 5 minutes. Drain TCE from beaker.

This step removes organic contaminations (such as oil, grease or wax) from the wafer surface.

(3) Ultra-sonic clean in $~ 50 \mathrm{ml}$ ACE for 5 minutes. Drain ACE from beaker.

ACE in this step removes the TCE residue and acts as a further cleaning solvent.

(4) Ultra-sonic clean in $50 \mathrm{ml} \mathrm{ME} \mathrm{for} 5$ minutes. Drain ME from beaker. This step removes the ACE residue.

(5) Rinse with deionized (DI) water and blow dry with compressed nitrogen gas. 
This step removes the ME residue.

All used solvents should be disposed in the proper solvents waste can and should not be left in the fume hood to evaporate!

\subsubsection{Piranha cleaning}

Piranha cleaning was used to clean our silicon or SOI wafers before the photolithography step in the cleanroom of Nanotech West. This step removes organic contaminants and leaves a thin and clean $\mathrm{SiO}_{2}$ film on the $\mathrm{Si}$ surface. The cleaning procedure is

(1) Ultra-sonic clean in $\sim 50 \mathrm{ml}$ ACE for 5 minutes.

(2) Rinse with DI water.

(3) Piranha cleaning $\left(\mathrm{H}_{2} \mathrm{SO}_{4}: \mathrm{H}_{2} \mathrm{O}_{2}=3-4: 1\right) @ 110^{\circ} \mathrm{C}-130^{\circ} \mathrm{C}$ or room temperature for 5-10 minutes.

(4) DI water rinse and $\mathrm{N}_{2}$ blow dry.

\subsubsection{Four-chemical cleaning}

For silicon oxide growth, silicon wafers with a diameter $\leq 3$ inches are cleaned using four-chemical clean which is an industry standard and is a sequence of four chemical solutions cleaning. This cleaning process was done in the 
cleanroom of Nanotech West. In this process, three hotplates are used to heat solutions, three high-purity fused silica (commonly known as quartz) 3-inch dishes are used for Piranha cleaning, SC(Standard Cleaning)1 and SC2 cleaning, and a high purity PTFE (polytetrafluoroethylene) dish is used for the HF dip. The cleaning procedure is

(1) Piranha cleaning $\left(98 \% \mathrm{H}_{2} \mathrm{SO}_{4}: \mathrm{H}_{2} \mathrm{O}_{2}=4: 1\right) @ 110^{\circ} \mathrm{C}-130^{\circ} \mathrm{C}$ for 310 minutes. This step removes organic contaminants.

(2) DI water rinse and $\mathrm{N}_{2}$ blow dry.

(3) HF dip (DI water: 49\% HF = 50:1 ) @ room temperature for 30-60 seconds. This step removes the $\mathrm{SiO}_{2}$ layer on the wafer and leaves a fresh silicon surface.

(4) DI water rinse and $\mathrm{N}_{2}$ blow dry.

(5) $\mathrm{SC} 1$ clean ( DI water : $29 \% \mathrm{NH}_{4} \mathrm{OH}: \mathrm{H}_{2} \mathrm{O}_{2}=20: 4: 1$ ) @ $65^{\circ} \mathrm{C}-$ $80^{\circ} \mathrm{C}$ for $3-10$ minutes. Also called RCA1 or APM. This procedure removes particulate contaminants, and desorbs trace metals ( $\mathrm{Au}, \mathrm{Ag}$, $\mathrm{Cu}, \mathrm{Ni}$, etc.). Some metals (e.g. Al) are insoluble in this oxidizing, highly basic solution and tend to precipitate on the surface of Si wafers. After this SC1 clean, the wafer surface is contaminated by Fe ions and is rough.

(6) DI water rinse and $\mathrm{N}_{2}$ blow dry. 
(7) $\mathrm{SC} 2$ Clean (DI Water : $\left.37 \% \mathrm{HCl}: 30 \% \mathrm{H}_{2} \mathrm{O}_{2}=5: 1: 1\right) @ 65^{\circ} \mathrm{C}-$ $80^{\circ} \mathrm{C}$ for 90 seconds. Also called RCA2 or HPM. This procedure dissolves alkali ions and hydroxides of $\mathrm{Al}^{3+}, \mathrm{Fe}^{3+}, \mathrm{Mg}^{3+}$.

(8) DI water rinse and $\mathrm{N}_{2}$ blow dry.

This method also consumes $\sim 20 \mathrm{~nm}$ silicon because the SC1 clean step cleans the silicon surface by etching some silicon material away. So it is not good for cleaning SIMOX SOI wafers with a thin silicon film.

\subsection{Photolithography}

In our experiments, photolithography was used to define areas where we want to deposit metal contacts or etch away the exposed silicon film of SOI wafers. For metal contact deposition, we tried two methods: lift-off with duallayer resists and lift-off with single layer resist. The lift-off process with duallayer resists (explained in the next section) was the first method we tried but we found that we couldn't see BEEM signals on most of the samples fabricated using this method. We thought the reason may be that there was still some lift-off resist (LOR) 2A left on the wafer surface after the photolithography process. Then we tried a lift-off process with single layer resist and we found this worked well for us. Photolithography was done in the cleanroom of Nanotech West. 
The spin coating and hotplate baking of photoresist films were done using a Cost Effective Equipment (CEE) 100CB spin coater with programmable spin, ramp, bake and exhaust sequences, and auto-dispense capabilities with syringe or cartridge. The alignment and UV exposure were done using an EV Group EV620 Mask Aligner with accuracy to 0.5 microns and resolution to 0.7 microns.

\subsubsection{Lift-off with dual-layer resists.}

In this method, first one layer of MicroChem LOR 2A is coated on the wafer and soft-baked (Figure 5.2(a)). Then one layer of Shipley Microposit photoreist S1813 is coated on top of the LOR 2A layer and soft-baked (Figure 5.1.a). After UV exposure (Figure 5.2(b)), the sample is insert into MF-319 developer and because LOR 2A develops isotropically an undercut will form (Figure 5.1(c)). This should result in a discontinuous metal film during evaporation (Figure 5.1(d)) and clear edge profiles after lift-off (Figure 5.1(e)) [58]. The procedure detail is

(1) Clean the wafer using Piranha cleaning for 20minutes.

(2) Bake at $200{ }^{\circ} \mathrm{C}$ for 30 minutes using a hot plate to remove moisture.

(3) Coat with LOR 2A (using Program \#9 in the spin coater).

Final speed $=3000 \mathrm{RPM}$ and time $=45$ seconds.

The LOR thickness is about 200nm. 

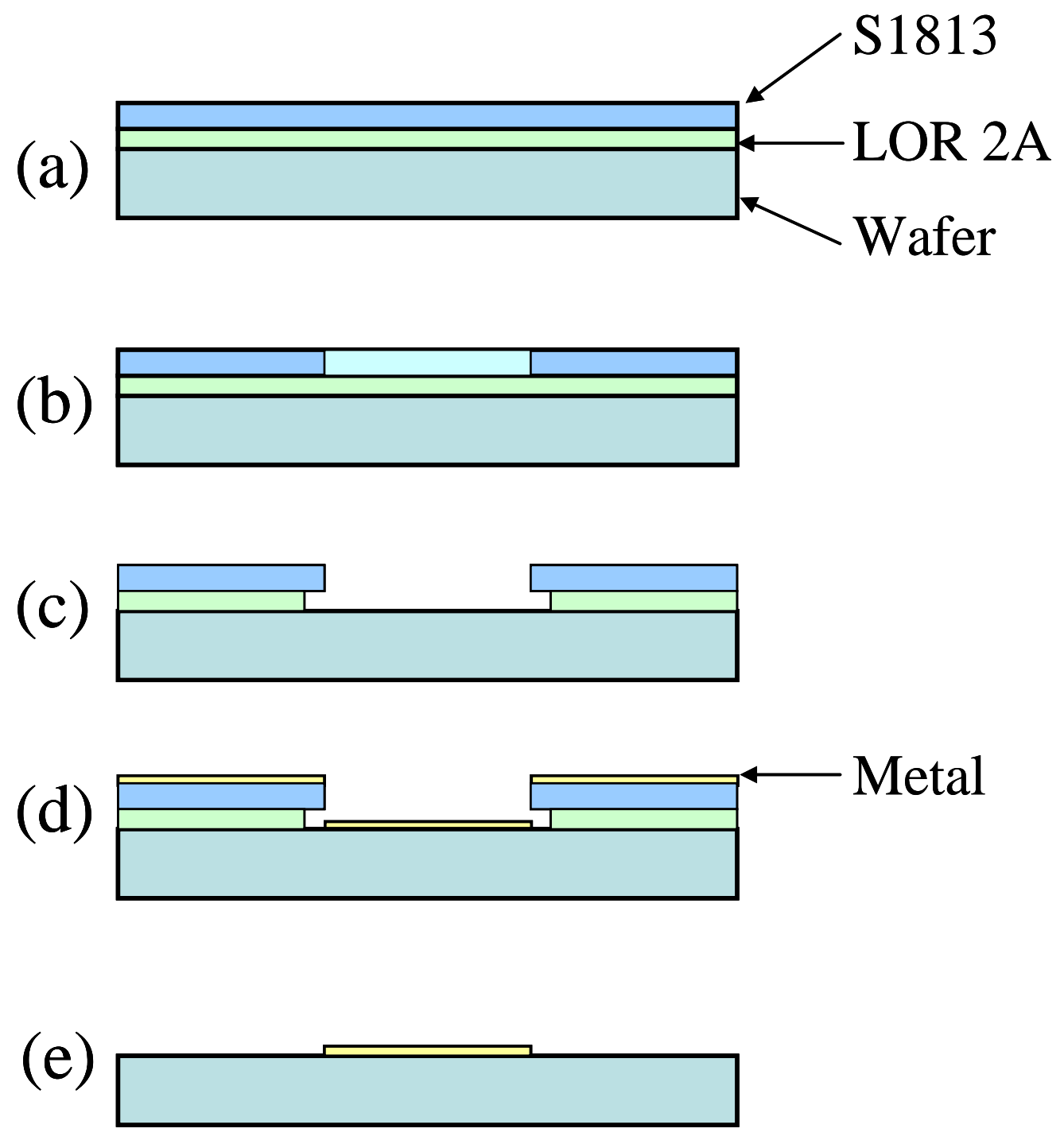

Figure 5.2: Lift-off process with dual-layer resists: (a) spin-coat and soft-bake LOR 2A and S1813; (b) Expose to UV; (c) Develop LOR 2A and S1813; (d) Metal deposition; and (e) Lift-off. 
(4) Prebake at $190{ }^{\circ} \mathrm{C}$ for 15 minutes using a hot plate.

(5) Coat with photoresist S1813 (using Program \#4 in the spin coater).

Final speed $=3000 \mathrm{RPM}$ and time $=60$ seconds .

The LOR thickness is about $1.4 \mathrm{um}$.

(6) Softbake at $115^{\circ} \mathrm{C}$ for 1 minute.

(7) Expose to UV for 6 seconds.

(8) Develop in MF-319 for 2 minutes.

(9) Metal deposition.

(10)Lift-off using NMP and Acetone.

\subsubsection{Lift-off with single layer resist.}

In this method, only one layer photoresist is used: Shipley S1813. Since there is no undercut, a continuous metal film may form during metal evaporation and this may result in ragged edges (Figure 5.3). These ragged edges may be a problem for other researchers but they are not a problem for us since we usually measure STM/BEEM away from the contact edge. The procedure detail is

(1) Clean the wafer using Piranha cleaning for 20 minutes.

(2) Coat with photoresist S1813 (using Program \#4 in the spin coater). Final speed $=3000 \mathrm{RPM}$ and time $=60$ seconds . 

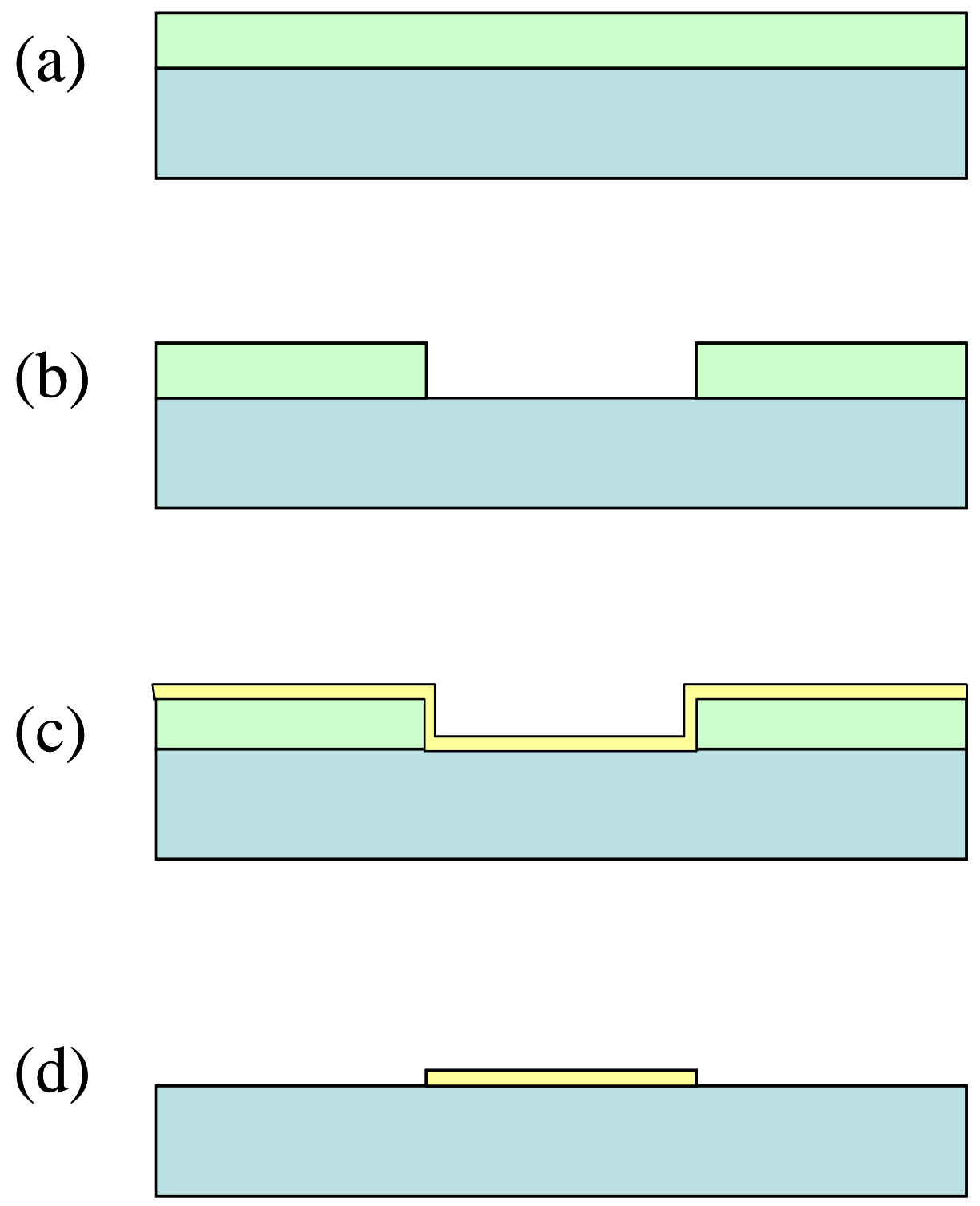

Figure 5.3: Lift-off process with single-layer resists: (a) spin-coat and soft-bake S1813; (b) Expose to UV and develop S1813 in MF-319; (c) Metal deposition; and (d) Lift-off. 
The LOR thickness is about $1.4 \mathrm{um}$.

(3) Softbake at $115^{\circ} \mathrm{C}$ for 1 minute.

(4) Expose to UV for 5-6 seconds.

(5) Develop in MF-319 for 1 minute.

(6) Metal deposition.

(7) Lift-off using Acetone.

\subsection{Dry etching}

Because current leakage paths sometimes exist in the BOX of SIMOX SOI wafer, we etched the SOI film to small rectangles to reduce the possibility that our sample structure has substantial leakage to the substrate. We used plasma etching or reactive ion etching (RIE) to etch the SOI film into small $(\sim 3.5 \mathrm{~mm} \times$ $0.9 \mathrm{~mm}$ ) rectangles. In these etching processes, photoresist S1813 was used as a mask, and was pattered using the method discussed in Section 5.3.2 with steps (a) and (b). The photoresist $\mathrm{S} 1813$ was hard-baked at $115^{\circ} \mathrm{C}$ for 30 minutes in the BlueM Oven \#1.

\subsubsection{Plasma etching}

The equipment we used is Lam 590 Oxide Etcher in Nanotech West. The recipe is

Gasses: $\mathrm{O}_{2}+\mathrm{He}+\mathrm{CF}_{4}=8 \mathrm{sccm}+10 \mathrm{sccm}+133 \mathrm{sccm}$; 
Pressure: 425 mTorr;

Power: 300W;

Gap between electrodes: $1.2 \mathrm{~cm}$;

Time: $100 \mathrm{~s}$.

After this plasma etching, for SIMOX SOI (35nm) samples, about $130 \mathrm{~nm}$ of the original $150 \mathrm{~nm} \mathrm{SiO} 2$ is left. For this piece of equipment all of the above parameters are controlled by the user. The details of this recipe are listed in Table 5.1 .

\begin{tabular}{|c|c|c|c|c|c|}
\hline & Step 1 & Step 2 & Step 3 & Step 4 & Step 5 \\
\hline $\begin{array}{c}\text { Pressure } \\
(\mathrm{mT} T o r r)\end{array}$ & 425 & 425 & 425 & 0 & 0 \\
\hline RF Power(W) & 0 & 0 & 300 & 0 & 0 \\
\hline Gap(cm) & 1.2 & 1.2 & 1.2 & 1.2 & 1.35 \\
\hline $\mathrm{O}_{2}(\mathrm{sccm})$ & 8 & 8 & 8 & 0 & 0 \\
\hline $\mathrm{He}(\mathrm{sccm})$ & 10 & 10 & 10 & 50 & 0 \\
\hline $\mathrm{CF}_{4}(\mathrm{sccm})$ & 133 & 133 & 133 & 0 & 0 \\
\hline $\begin{array}{c}\text { Other } \\
\text { chemicals } \\
(\mathrm{sccm})\end{array}$ & 0 & 0 & 0 & 0 & 0 \\
\hline $\begin{array}{c}\text { COMPL } \\
\text { Time }\end{array}$ & $10 \mathrm{~s}$ & $\begin{array}{c}\text { Time/stability } \\
15 \mathrm{~s}\end{array}$ & $\begin{array}{c}\text { Time } \\
100 \mathrm{~s}\end{array}$ & $\begin{array}{c}\text { Time } \\
15 \mathrm{~s}\end{array}$ & RECIPE \\
\hline
\end{tabular}

Table 5.1: Details of the recipe for plasma etching $35 \mathrm{~nm}$ SIMOX SOI wafers. 


\subsubsection{Reactive ion etching}

The equipment we used was the Technics Micro-RIE (Series 800-II) in Nanotech West. Because the gas lines connected to this equipment are switched between $\mathrm{CF}_{4}+\mathrm{O}_{2}$ or $\mathrm{CHF}_{3}+\mathrm{O}_{2}$ depending on the users' requests, we developed one recipe for each of these two connections. These two recipes are

(1) Gasses: $\mathrm{CF}_{4}+\mathrm{O}_{2}=30 \mathrm{sccm}+10 \mathrm{sccm}$;

Power $=80-90 \mathrm{~W}$;

Pressure $\approx 233 \mathrm{mTorr}$

Time $=150 \mathrm{~s}$.

About 102-114 $\mathrm{nm}$ of the original $150 \mathrm{~nm} \mathrm{SiO}_{2}$ BOX layer is left for the $35 \mathrm{~nm}$ SIMOX SOI samples.

(2) Gasses: $\mathrm{CHF}_{3}+\mathrm{O}_{2}=30 \mathrm{sccm}+10 \mathrm{sccm}$;

Power $=85-92 \mathrm{~W}$;

Pressure $\approx 233 \mathrm{mTorr}$

Time $=150 \mathrm{~s}$.

About $110-120 \mathrm{~nm} \mathrm{SiO}_{2}$ is left for the $35 \mathrm{~nm}$ SIMOX SOI samples.

For this piece of equipment, only the flow rate of the reactive chemicals, the RF power and the process time can be controlled by users. The RF power fluctuates 
during the etching so a power range was shown in the above recipes instead of a specific number.

\subsection{Wet etching}

Before the silicon wafer is transferred into the evaporation chamber for metal depositions, we need to remove the native oxide on the silicon wafer. This is done by a $20-30$ seconds dip in a diluted hydrofluoric acid solution (10:1 or 7:1 buffered oxide etch) and then a 1 minute DI water rinse. $49 \% \mathrm{HF}$ is not good since it attacks the photoresist S1813. After this HF dip, the silicon wafer is transferred into the evaporation chamber very quickly and the chamber is pumped down just after this transfer to avoid oxide growth. After the HF dip, the silicon surface is passivated with $\mathrm{H}-\mathrm{Si}$ bonds which make the surface oxidation rate very slow [59].

\subsection{Metal evaporation}

In our ambipolar BEEM experiments, we need to do four metal depositions: one for ohmic contacts on the backside of the wafers, two for ohmic contacts on the top SOI film, and one for the Schottky contacts on the top SOI film. The first three contacts were deposited using the e-beam evaporator DV(Denton Vacuum)-502A in Nanotech West, and the Schottky contacts were deposited using our own custom-made thermal evaporator. 
For making a Au/GaAs reference sample, the native oxide on GaAs is removed by dipping in a $\mathrm{NH}_{4} \mathrm{OH}: \mathrm{DI}=1: 1$ solution for 1 minute followed by a 1 minute rinse in DI water and $\mathrm{N}_{2}$ blow dry.

\subsubsection{E-beam evaporation}

In this evaporation process, an electron beam is used to heat the source material and causes it to evaporate.

Using the e-beam evaporator DV(Denton Vacuum)-502A in Nanotech West, we deposited the ohmic contacts ( $\mathrm{Au}$, Ti and Pt) on the backside and on the SOI film. The deposition rate is around $0.3-0.5 \AA / s$, and the pressure before evaporation is around $1-4 \times 10^{-6}$ Torr. The source materials are from Kurt J Lesker: $\mathrm{Au}($ purity $=99.99 \%)$, Ti $($ purity $=99.995 \%)$ and $\mathrm{Pt}($ purity $=99.99 \%)$. The deposited film thickness is monitored by a crystal monitor.

\subsubsection{Thermal evaporation of Schottky contacts}

The Schottky contacts ( $\mathrm{Au} / \mathrm{Si}$ and $\mathrm{Cu} / \mathrm{Si})$ are deposited using our own thermal evaporator [60]. In this thermal evaporator, the metal sources ( $\mathrm{Au}$ and $\mathrm{Cu}$ ) are placed in two tungsten boats which are separated with a piece of plate to avoid contaminating each other. The metal source is heated using a high-power current source until the metal starts to evaporate. The deposition rate is around $0.3-0.5 \AA / \mathrm{s}$.

The pressure before evaporation is typically $\leq 1-2 \times 10^{-7}$ Torr, which can be 
achieved after 4-5 hours of pumping. Also the evaporator allows the heating or cooling of samples during evaporation to modify the grain structure of the deposited metal [46]. The metal contacts can be pattered using photolithography or a metal shadow mask with an array of 450um diameter holes. The deposited film thickness is monitored by a crystal monitor. The source materials we used are $\mathrm{Au}($ purity $=99.999 \%)$ from Kurt J Lesker and oxygen-free $\mathrm{Cu}($ purity $=99.995 \%)$ from Alfar Aesar.

\subsection{Fabrication steps for one complete SOI sample}

Here, we give a general procedure for fabricating a complete SOI sample without repeating the details of each process step.

(1) Cleave a piece of SOI wafer with a size of $1.1 \mathrm{~cm} \times 1.5 \mathrm{~cm}$.

(2) Clean it using ultrasonic cleaning in Acetone and then clean it using piranha cleaning.

(3) Evaporate 100nm Au on the backside of the wafer.

(4) Do photolithography and plasma or RIE etch the silicon islands.

(5) Do photolithography and evaporate ohmic contacts and lift-off.

(6) Scribe lines using a diamond scribe for the cleaving in Step (8). The goal for this step is to protect the metal film of the Schottky contacts and the SOI film during the cleaving in Step (8). By scribing the 
cleaving lines in this step, the SOI wafer can be broken into four pieces before the liftoff Step (9), so the SOI wafer is covered with 1.4um photoresist. This will protect the metal film of the Schottky contacts and the SOI film.

(7) Do photolithography and evaporate $\mathrm{Au}$ or $\mathrm{Cu}$ Schottky contacts.

(8) Break this piece of wafer into four pieces. The size of each cleaved sample should be smaller than $5 \mathrm{~mm} \times 5 \mathrm{~mm}$ because the space on the STM sample holder is $5 \mathrm{~mm} \times 5 \mathrm{~mm}$. According to my experience, it is better to make the sample size around $4.5 \mathrm{~mm} \times 4.5 \mathrm{~mm}$, so the sample size is not too small and it is easy to place it on the sample holder.

(9) Lift-off in acetone and ultra-sonic clean in methanol

(10) Rinse in DI water and blow dry with $\mathrm{N}_{2}$ gas. 


\section{CHAPTER 6}

\section{AMBIPOLAR BEEM MEASUREMENTS}

In this chapter, we report measurements using ballistic electron/hole emission microscopy (BEEM/BHEM) on device structures fabricated SOI wafers to investigate how the local electron (n-type) and hole (p-type) Schottky barrier heights (i.e., local valence-maximum and conduction-minimum band energies at the metal/Si interface) are modified by an applied gate field. We demonstrate the first nmresolution ambipolar $n-B E E M / p-B H E M$ measurements performed at the same location on $\mathrm{Au} / \mathrm{Si}$ and $\mathrm{Cu} / \mathrm{Si}$ Schottky contacts at temperatures down to $80 \mathrm{~K}$. The local Schottky barrier heights for electrons and holes at particular locations were measured and, when corrected for image force lowering, were found to sum to nearly the same value (approximately the silicon bandgap). While the individual $\mathrm{n}$ - and $\mathrm{p}$ SBHs varied by more than $200 \mathrm{meV}$ between the $\mathrm{Au} / \mathrm{Si}$ and $\mathrm{Cu} / \mathrm{Si}$ contacts, for a given sample they sum to within $15 \mathrm{meV}$ of the same value, indicating that the 
individual variations are due to variations in a local surface dipole as compared with tip effects or variations in local composition. This method could be a platform for studying the band gap of different epitaxial layers and band offset in heterostructures.

\subsection{Previous BEEM results on $\mathrm{Au} / \mathrm{Si}$ and $\mathrm{Cu} / \mathrm{Si}$ contacts}

Before describing the details of our ambipolar BEEM measurements, we briefly review previous BEEM/BHEM results on metal/Si interfaces. Since the Schottky barriers in our ambipolar BEEM experiments are $\mathrm{Au} / \mathrm{Si}(100)$ and $\mathrm{Cu} / \mathrm{Si}(100)$, in this section we only discuss previous BEEM/BHEM results on $\mathrm{Au} / \mathrm{Si}(100)$ and $\mathrm{Cu} / \mathrm{Si}(100)$.

\subsection{1 $\mathrm{Au} / \mathrm{Si}(100)$}

In their initial work, Kaiser and Bell measured the barrier height of $\mathrm{Au} / \mathrm{n}$ $\operatorname{Si}(100)$ ( doping $\cong 2 \times 10^{15} / \mathrm{cm}^{3}$ ) using BEEM at room temperature [47].The average Schottky barrier height they measured was $0.82 \mathrm{eV}$ determined from BEEM spectrum fitted with a thermally-broadened square law ( $n=2$, see Section 4.3). At $\mathrm{T}=77 \mathrm{~K}$, Bell et al. measured the Schottky barrier heights of $\mathrm{Au} / \mathrm{n}-\mathrm{Si}(100)$ (doping $n=$ $\left.2 \times 10^{15} / \mathrm{cm}^{3}\right)$ and $\mathrm{Au} / \mathrm{p}-\mathrm{Si}(100)$ (doping $p=3 \times 10^{15} / \mathrm{cm}^{3}$ ) using BEEM/BHEM [61-63]. The barrier heights were determined to be $0.82 \mathrm{eV}$ for $n$-Si and $0.35 \mathrm{eV}$ for $p$-Si using a square law. The measured Schottky barrier height using BEEM by different groups was found to vary slightly with sample preparation and measurement conditions, in the range from 0.75 to $0.87 \mathrm{eV}[37,47,61-67]$. 
Figure 6.1 shows our measured BEEM/BHEM spectra on $\mathrm{Au} / \mathrm{n}-\mathrm{Si}(100)$ and $\mathrm{Au} / \mathrm{p}-\mathrm{Si}(100)$ at $\mathrm{T}=80 \mathrm{~K}$, using the equipment discussed in Section 4.4. The red curves are the experimental results and the blue ones are the fits using the BK model. The average measured barrier height is $0.818(2) \mathrm{eV}$ for $n$-Si and $0.332(2) \mathrm{eV}$ for $p$-Si. The doping of the Si wafer used in these experiments is $n \cong 0.2-1.0 \times 10^{16} / \mathrm{cm}^{3}$ for the $n$-Si and $p \cong 3 \times 10^{16} / \mathrm{cm}^{3}$ for the $p$-Si.

\subsection{2 $\mathrm{Cu} / \mathrm{Si}(100)$}

Labella et al. measured the Schottky barrier height of $\mathrm{Cu} / \mathrm{n}-\mathrm{Si}(100)$ (doping $\cong$ $0.3-4.0 \times 10^{15} / \mathrm{cm}^{3}$ ) using BEEM at $\mathrm{T}=80 \mathrm{~K}$ and the value they obtained was 0.64 $\mathrm{eV}[68]$. Banerjee et al. measured the Schottky barrier height of $\mathrm{Cu} / \mathrm{p}-\mathrm{Si}(100)$ using $\mathrm{BHEM}$ at $\mathrm{T}=150 \mathrm{~K}$ and the value they obtained was $0.48 \mathrm{eV}[69]$.

\subsection{Ambipolar BEEM studies}

For the BEEM/BHEM measurements mentioned in Section 6.1, the Schottky contacts were fabricated by depositing $\mathrm{Au}$ or $\mathrm{Cu}$ on regular n-type or p-type silicon substrate. Due to the band bending inside the silicon substrate, only BEEM can be performed on $\mathrm{Au}(\mathrm{Cu}) / \mathrm{n}-\mathrm{Si}$ contacts and only BHEM can be performed on $\mathrm{Au}(\mathrm{Cu}) / \mathrm{p}$ Si contacts. For an $\mathrm{Au}(\mathrm{Cu}) / \mathrm{n}-\mathrm{Si}$ contact, starting from the metal/silicon interface, the energy bands bend downward towards into the silicon as shown in Figure 6.2 (a). In this case, if hot electrons are injected across the metal/silicon interface, they will be 

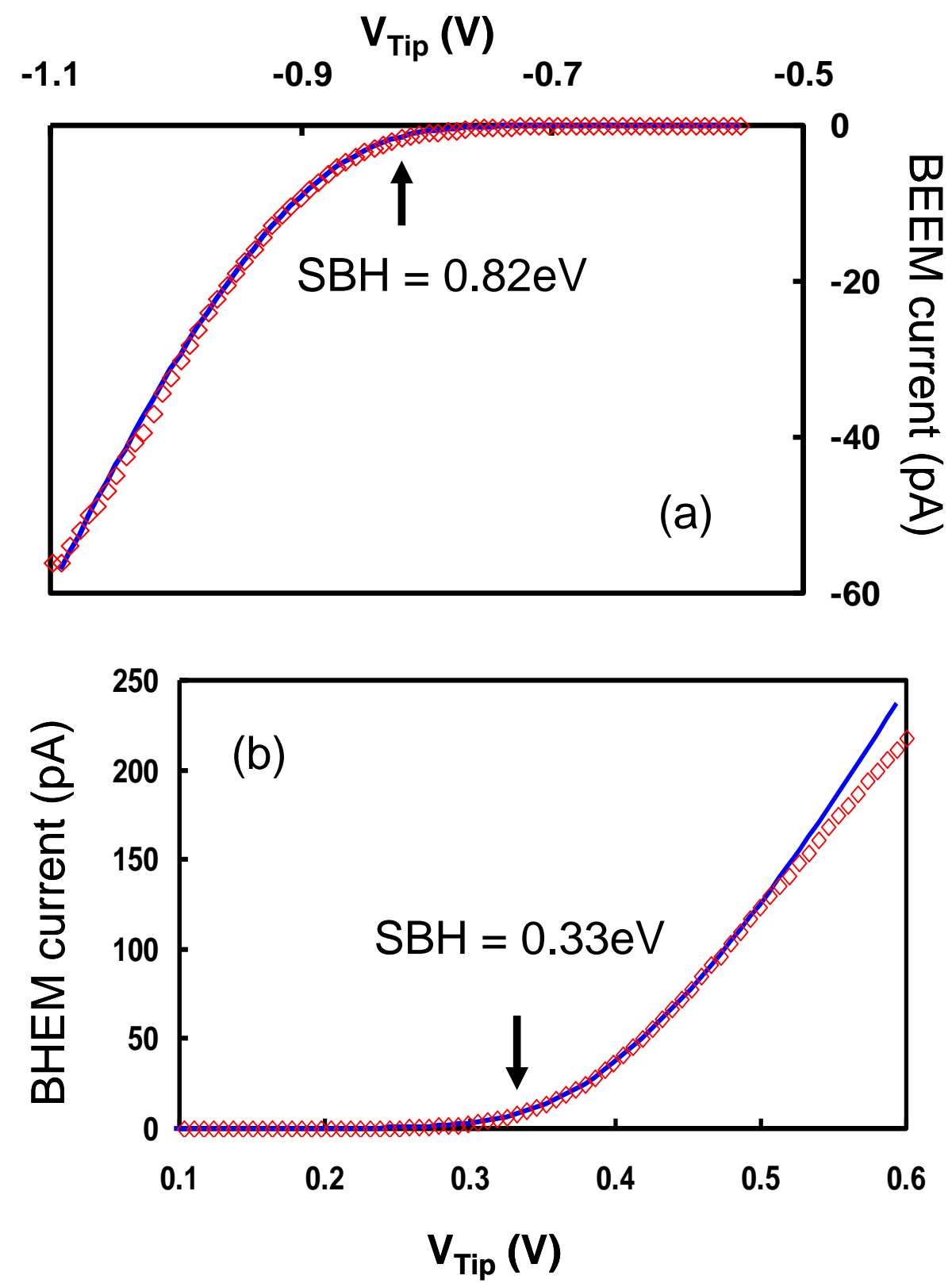

Figure 6.1: BEEM/BHEM spectrum measured on (a) Au/n-Si(100) and (b) Au/p$\mathrm{Si}(100)$ at $\mathrm{T}=80 \mathrm{~K}$. Red - experimental results and blue - fitted results using the $\mathrm{BK}$ model. 
swept away from the interface and into the substrate by the electric field due to the band bending, so they can be collected as BEEM current by the ohmic contact on the substrate. However, if hot holes are injected across the metal/silicon interface, they will be swept back towards the interface and back into the metal by the electric field because they have positive charge, so they cannot be collected as BHEM current by the ohmic contact at the other end of the substrate. So for an $\mathrm{Au}(\mathrm{Cu}) / \mathrm{n}-\mathrm{Si}$ contact, only electrons can be collected, i.e. only BEEM measurements can be performed and only the Schottky barrier height for electrons can be measured. For an $\mathrm{Au}(\mathrm{Cu}) / \mathrm{p}-\mathrm{Si}$ contact, similar argument can show that only holes can be collected, so only BHEM measurements can be performed and only the Schottky barrier height for holes can be measured. Therefore, for conventional BEEM or BHEM measurement, separate samples have to be used to measure the Schottky barrier heights for electrons and holes. This makes it difficult to directly compare the electron- and hole-SBHs.

Here we will demonstrate the first nm-resolution ambipolar $n$-BEEM/pBHEM measurements which can measure the local Schottky barrier heights for electrons and holes at the same location on the same metal/silicon contact, allowing for the first time a direct comparison between the electron- and hole-SBHs on a particular sample. 


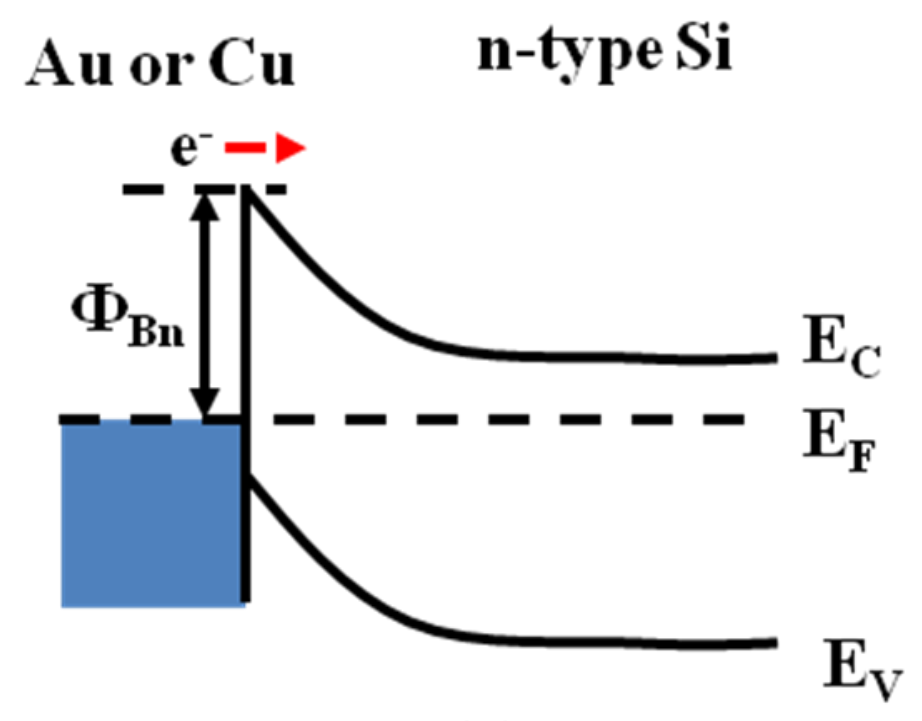

(a)

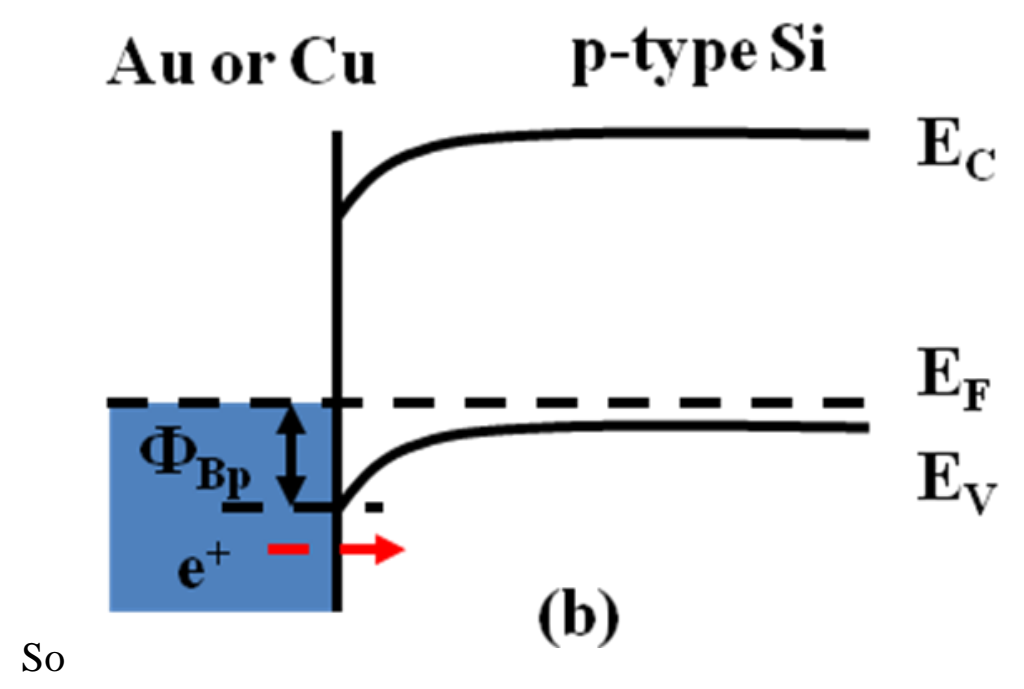

Figure 6.2: Energy band diagrams of (a) Metal/n-Si and (b) Metal/p-Si. 


\subsubsection{Sample geometry and basic idea of Ambipolar BEEM}

Figures 6.3 and 6.4 illustrate the basic idea of how to measure the local Schottky barrier heights for electrons and holes at the same location on the same metal/silicon contact using SOI structures.

The schematic diagram of sample geometry and wiring for our ambipolar BEEM measurements is shown in Figure 6.3. Electrons or holes are injected from the Schottky contact, and then they conduct laterally and are collected at the ohmic contact. The electrode on the backside of the substrate is used to apply a back-gate voltage which can make the same metal/silicon Schottky barrier either effectively ntype (Figure 6.4(a)) or p-type (Figure 6.4(b)) depending on the polarity of the substrate gate bias. Since the top silicon film has a doping of $\sim 1 \times 10^{15} / \mathrm{cm}^{3}$ and the corresponding bulk depletion width is $\sim 1 \mu \mathrm{m}$, the dopant-related band bending in the $35 \mathrm{~nm}$-thick silicon film should be negligible. Then if a positive substrate bias is applied to the substrate, the energy bands in the top silicon film will bend downward (similar to an n-type Schottky barrier) as shown in Figure 6.4(a). If the applied positive bias is large enough so that the conduction band minimum approaches the silicon film Fermi level $E_{F, S i}$ at the $\mathrm{Si} / \mathrm{SiO}_{2}$ interface, then electrons will accumulate near the $\mathrm{Si} / \mathrm{SiO}_{2}$ interface and allow the $\mathrm{Si}$ film to become laterally conductive. When the silicon film is conducting enough, hot electrons injected from a negatively-biased tip can be swept to the $\mathrm{Si} / \mathrm{SiO}_{2}$ interface, then conduct laterally and be collected as BEEM current at the ohmic contact. This allows BEEM measurements to be performed and the Schottky barrier height for electrons can be determined. In contrast, 


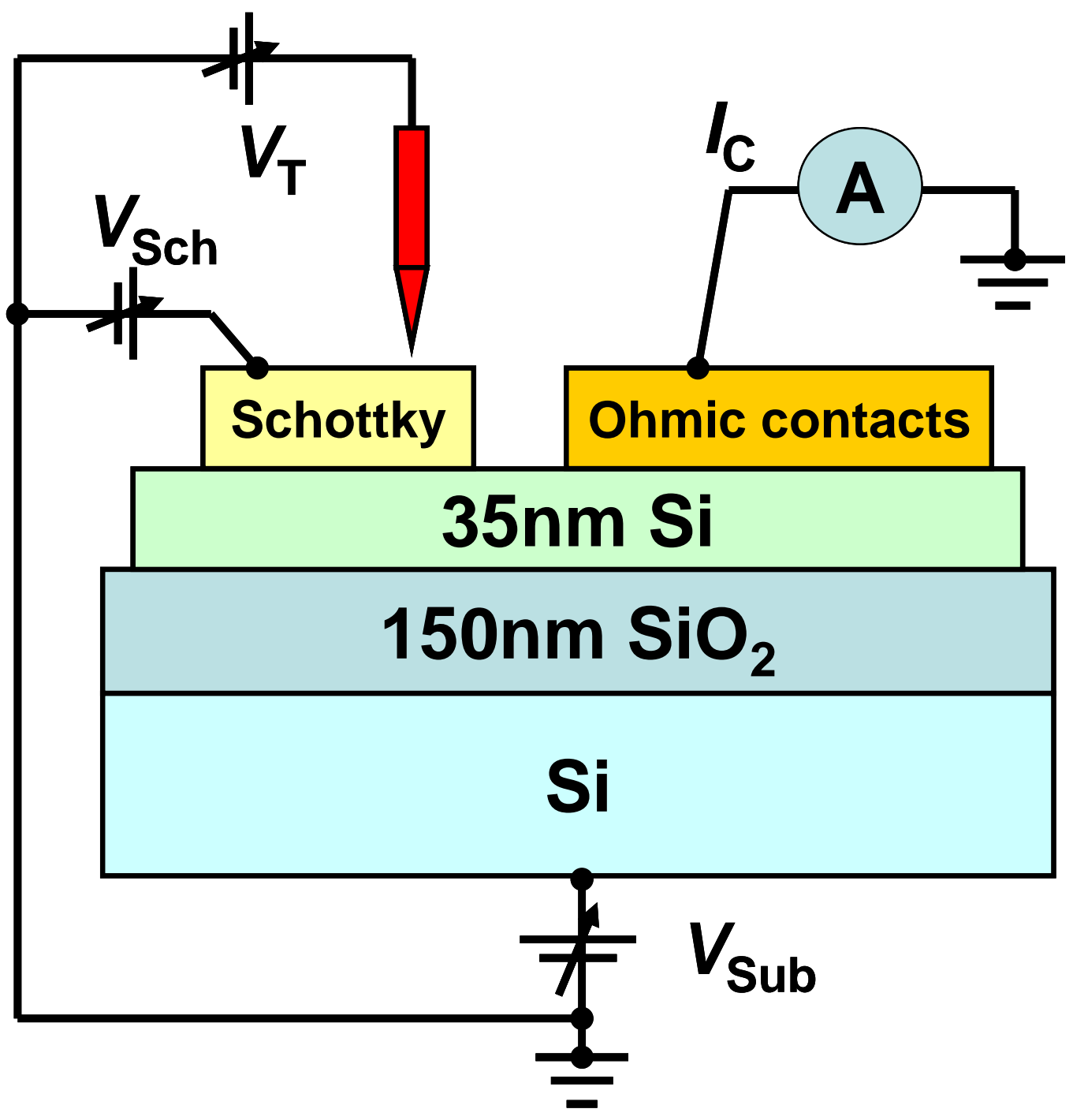

Figure 6.3: Schematic diagram of sample geometry and wiring for ambipolar BEEM measurements. 


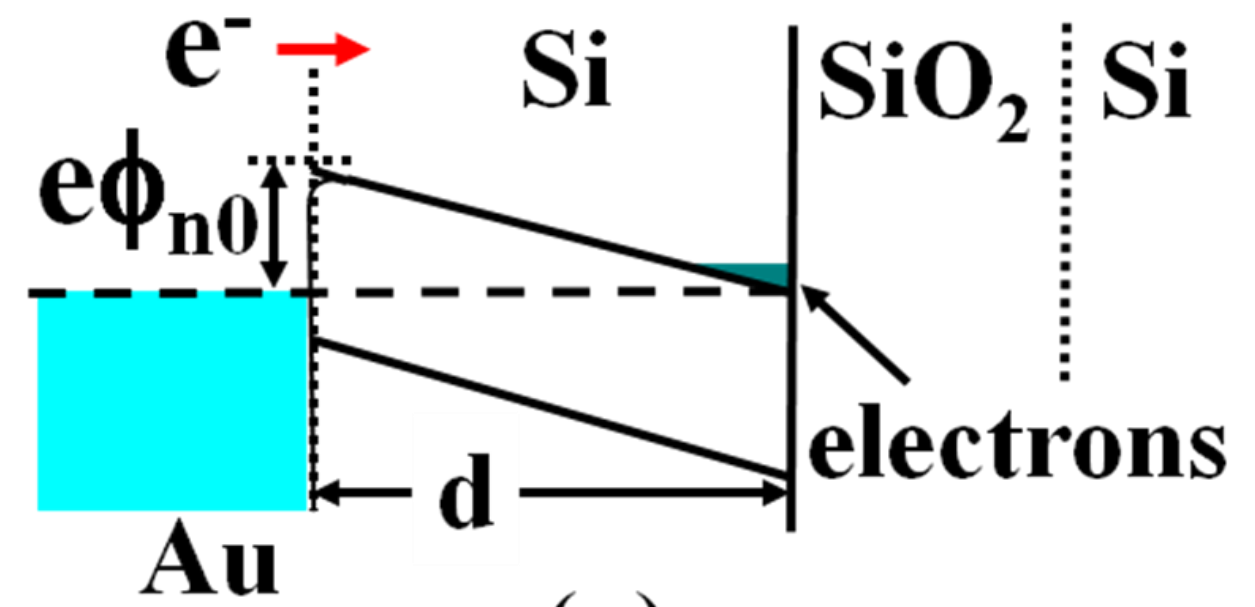

(a)

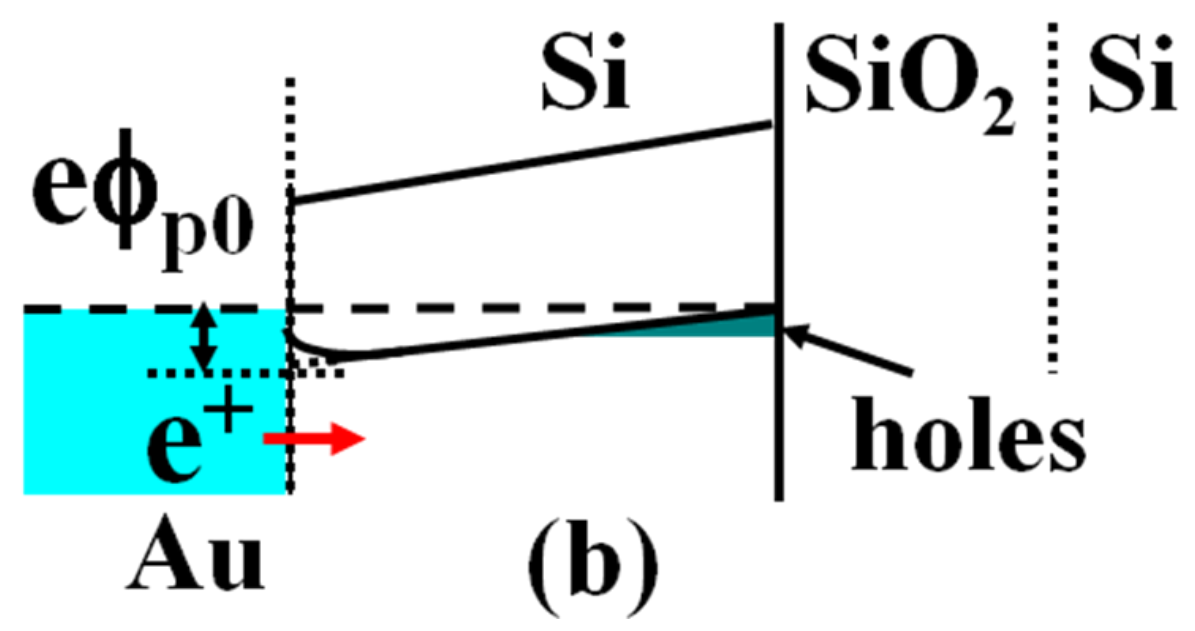

Figure 6.4: Energy band structures for Au/Si barriers (a) SOI sample with a positive substrate bias, and (b) SOI sample with a negative substrate bias. Here e $\phi_{\mathrm{n} 0}$ and $\mathrm{e} \phi_{\mathrm{p} 0}$ are intrinsic Schottky barrier heights. 
if a negative substrate bias is applied to the substrate, the energy bands in the top silicon film will bend upward (similar to a p-type Schottky barrier) as shown in Figure 6.4(b). If the applied positive bias is large enough so that the valence band maximum approaches the silicon film Fermi level $E_{F, S i}$ at the $\mathrm{Si}_{2} \mathrm{SiO}_{2}$ interface, then holes will accumulate near the $\mathrm{Si} / \mathrm{SiO}_{2}$ interface. When the silicon film is conducting enough due to hole accumulation, holes injected from a positively-biased tip can be swept to the $\mathrm{Si} / \mathrm{SiO}_{2}$ interface, then conduct laterally and be collected as BHEM current at the ohmic contact. This allows BHEM measurements to be performed and the Schottky barrier height for holes can be determined. Therefore, using this SOI structure, the local Schottky barrier height for both electrons and holes can be measured at the same location on the same metal/silicon contact.

\subsubsection{Ambipolar BEEM measurement results}

Before describing the details of the ambipolar BEEM measurement results, the general procedure of how to get the barrier heights from BEEM/BHEM measurements is first discussed. In the following discussion, we use BEEM measurement as an example and note that an analogous procedure applies for BHEM measurements. During BEEM spectra measurements, the scan area was set to be a square whose sides had a size of 300-400nm. In a particular scan, a BEEM spectrum was measured at about 10-20 locations which were randomly chosen. At each of these 10-20 locations, 10 BEEM IV curves were measured and these 10 curves were averaged to give one BEEM spectrum which was then fitted using BK model to give 
one local Schottky barrier height. On one Schottky contact, at least two groups of measurements were done: one with the tip voltage swept from low to high and the other with the tip voltage swept from high to low (to reduce the effect of time delay in BEEM IVs at low temperature). Then all of barrier heights measured with the tip voltage swept from low to high were averaged to give the Schottky barrier height $q \phi_{L-H}$, and all of barrier heights measured with the tip voltage swept from high to low were averaged to give the Schottky barrier height $q \phi_{H-L}$. Finally these two measured barrier heights $q \phi_{L-H}$ and $q \phi_{H-L}$ were averaged to give the average barrier height of the Schottky contact. And the standard deviation of measured barrier heights was also determined and was used to calculate the uncertainty in the average barrier height. Typical ambipolar BEEM spectra at $80 \mathrm{~K}$ with $\mathrm{V}_{\mathrm{Sch}}=0$ on a gated $\mathrm{Au}(\mathrm{Cu}) / \mathrm{Si}(\mathrm{SOI})$ contact are shown in Figure 6.5 (see Fig. 6.3 for the definition of $\left.\mathrm{V}_{\mathrm{Sch}}\right)$.

In Figure 6.5 (a), the blue curve is a typical BEEM spectrum measured at 80 $\mathrm{K}$ on a $\mathrm{Au} / \mathrm{Si}$ (SOI) contact with a positive gate bias of $7.0 \mathrm{~V}$ applied to the substrate and with the Ti/Si contact used as the ohmic contact to collect the BEEM current. For comparison, the red curve shows a typical BEEM spectrum for a regular $\mathrm{Au} / \mathrm{n}-\mathrm{Si}$ (doping $\sim 0.2-1.0 \times 10^{16} \mathrm{~cm}^{-3}$ ) also measured at $80 \mathrm{~K}$. The dotted curves are the experimental results and the solid lines are the corresponding Bell-Kaiser fits. For the $\mathrm{Au} / \mathrm{Si}(\mathrm{SOI})$ contact, the average barrier height was found to be $0.787(2) \mathrm{eV}$, while for the regular $\mathrm{Au} / \mathrm{n}-\mathrm{Si}$ contact, the average barrier was found to be $0.818(2) \mathrm{eV}$ (here the number inside the bracket is the uncertainty in the average). We see the Schottky 
Tip Voltage (V)
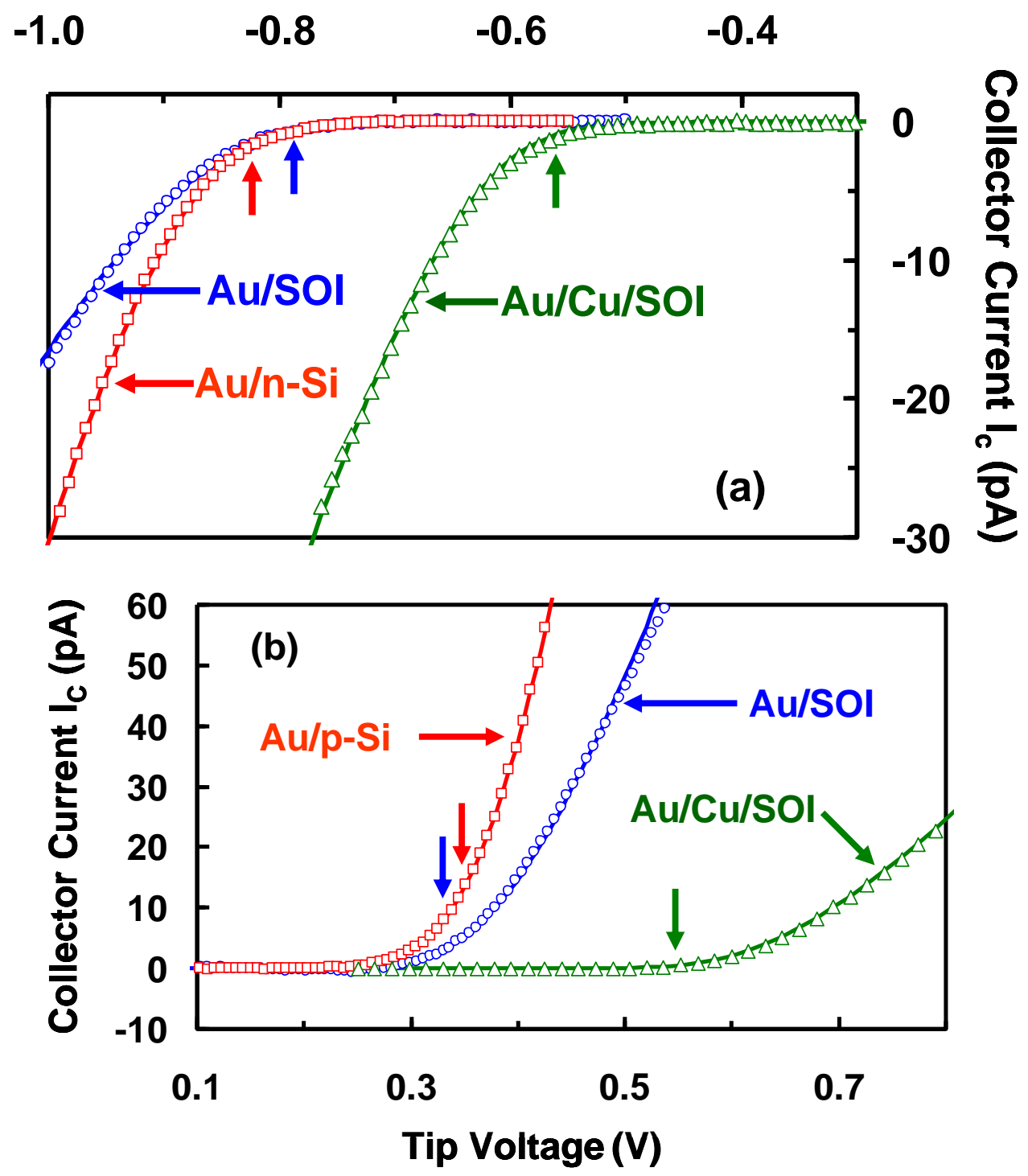

Figure 6.5: (a) BEEM and BHEM spectra at $\mathrm{T}=80 \mathrm{~K}$. Red ( $(\square)$, blue ( $(\circ)$, and green $(\Delta)$ data points correspond to regular $\mathrm{Au} / \mathrm{n}-\mathrm{Si}, \mathrm{Au} / \mathrm{SOI}$ and $\mathrm{Au} / \mathrm{Cu} / \mathrm{SOI}$. Solid lines are corresponding Bell-Kaiser fits. 
barrier height is $\sim 31 \mathrm{meV}$ lower on the gated SOI sample as compared with the Schottky barrier height on the regular silicon. This can be explained by the mechanism of image force lowering discussed in Section 3.3. To estimate the electric field inside the silicon film near the interface, we can assume that the conduction band minimum in the silicon film is very close to the Fermi level at the interface and the slope of the band bending is constant (due to the low doping of the Si film and the small $35 \mathrm{~nm}$ thickness of the Si film), so the electric field inside the silicon film near the interface can be estimated by

$$
E=\frac{\phi_{n 0}}{d}
$$

where $q \phi_{n 0}$ is the intrinsic Schottky barrier and $d$ is the silicon film thickness. From this the interface electric field in the gated SOI sample is estimated to be $\sim 2 \times 10^{5}$ V/cm. Using Equation (3.8), the image force lowering due to this field is found to be $\sim 49 \mathrm{meV}$ in the gated SOI sample. The interface electric field in the regular $\mathrm{Au} / \mathrm{n}-\mathrm{Si}$ sample can be estimated by

$$
E=\sqrt{\frac{2 q N_{D}}{\varepsilon_{s}}\left(V_{b i}-\frac{k_{B} T}{q}\right)},
$$


where $N_{D}$ is the doping of the silicon substrate, $\varepsilon_{s}=11.7 \varepsilon_{0}$ is the silicon permittivity and $V_{b i}$ is the built-in voltage inside the silicon [6]. The built-in voltage $V_{b i}$ and can be estimated by

$$
V_{b i}=q \phi_{n}-\left(E_{C}-E_{F}\right)
$$

where $\left(E_{C}-E_{F}\right)$ can be determined using Equation (2.6) [6]. For a phosphorous doped silicon with a doping level of $\sim 0.2-1.0 \times 10^{16} / \mathrm{cm}^{3},\left(E_{i}-E_{F}\right)$ is around $0.52 \mathrm{eV}$, so the depletion field can be estimated to be $\sim 4 \times 10^{4} \mathrm{~V} / \mathrm{cm}$. This will produce an image force lowering of $\sim 22 \mathrm{meV}$. The difference between these image force lowering is $\sim 27 \mathrm{meV}$ which is close to the difference between the measured Schottky barrier heights $\sim 31 \mathrm{meV}$.

We can also notice that the amplitude of the BEEM current is slightly smaller in the gated SOI sample than in the regular silicon sample. The smaller BEEM amplitude in the gated sample may result if some of the injected electrons cross back over the barrier into the Au film (and hence are not collected) as they move laterally towards the ohmic contact. And also because of the reflection due to the band structure of the BOX under the silicon film, the transmission coefficient $C(E, \boldsymbol{u})$ may be much smaller in the SOI sample than that in the regular sample.

We note that at lower gate bias the resistance of the Si film becomes much larger (due to reduced electron accumulation at the $\mathrm{Si} / \mathrm{SiO}_{2}$ interface - see Figure 6.4 (a) causing the BEEM current to be greatly reduced. At larger gate bias, the 
resistance of the Si film becomes smaller (due to greater electron accumulation) and the BEEM current becomes significantly noisier due to increased leakage between the $\mathrm{Au}$ and the electron accumulation layer at the $\mathrm{Si} / \mathrm{SiO}_{2}$ interface. As a consequence, the best BEEM signal-to-noise ratio was obtained for a substrate bias $V_{\text {sub }}$ in the range of 6 to $8 \mathrm{~V}$.

For hole-BHEM on the gated Schottky contact (blue curve in Figure 6.5(b)), a negative bias of $-17.0 \mathrm{~V}$ was applied to the substrate and Pt/Si contact was used as the ohmic contact to collect the BHEM current. The comparison red curve in Figure 6.5(b) is from a regular $\mathrm{Au} / \mathrm{p}-\mathrm{Si}$ (doping $\sim 3 \times 10^{16} / \mathrm{cm}^{3}$ ) contact at $80 \mathrm{~K}$. The measured average barrier height is $0.323(2) \mathrm{eV}$ for the gated SOI sample and 0.332(2) $\mathrm{eV}$ for the regular silicon sample. We can see the measured average barrier height is $\sim 9 \mathrm{meV}$ lower on the gated sample as compared with the regular Au/p-Si sample, and also the amplitude of the BHEM current is smaller on the SOI sample, most likely for the same reasons as discussed above for the n-type BEEM measurements. In the ptype case, the best signal-to-noise was found for the gate bias range of -16 to $-18 \mathrm{~V}$. We note that in general, the p-type Schottky zero-bias resistance was smaller than the n-type zero-bias resistance, resulting in noisier BHEM as compared to BEEM measurements.

Using the measured barrier heights and the mechanism of image force lowering, the intrinsic Schottky barrier height $q \phi_{n(p) 0}$ can be extracted selfconsistently from 


$$
q \phi_{n(p)}=q\left(\phi_{n(p) 0}-\sqrt{q E / 4 \pi \varepsilon_{s}}\right)
$$

where the electric field is determined by $E=\phi_{n(p) 0} / d$. The intrinsic barrier heights for $\mathrm{Au} / \mathrm{SOI}$ sample were determined to be $0.841(2) \mathrm{eV}$ for electrons and $0.359(2) \mathrm{eV}$ for holes. These sum to $1.200(3) \mathrm{eV}$ which is close to the expected Si bandgap $E_{\mathrm{g}}$ $\sim 1.17 \mathrm{eV}$ at $80 \mathrm{~K}$ [6]. The $\sim 30 \mathrm{meV}$ difference may be due to systematic errors in fitting the data or inaccurate estimates of the interface electric fields.

Similar measurements were also made using gated $\mathrm{Cu} / \mathrm{Si}$ Schottky contacts (green data points in Figure 6.5), which are expected to have a smaller n-type and a larger p-type Schottky barrier height as compared to Au/Si. Previous experiments on $\mathrm{Cu} / \mathrm{Si}$ contacts conducted by regular BEEM or BHEM reported a barrier height of $0.64(2) \mathrm{eV}$ for electrons at $80 \mathrm{~K}[68]$ and $0.48(3) \mathrm{eV}$ for holes at $\mathrm{T}=150 \mathrm{~K}$ [69], respectively. Using our gated $\mathrm{Cu} / \mathrm{Si}$ contacts, at $80 \mathrm{~K}$ we found a measured barrier height of $0.570(2) \mathrm{eV}$ for electrons and $0.551(7) \mathrm{eV}$ for holes respectively, which yield corresponding intrinsic barrier heights of $0.617(2) \mathrm{eV}$ and $0.597(7) \mathrm{eV}$ respectively, when self-consistently corrected of image force lowering using Equation (6.4). These sum to $1.214(4) \mathrm{eV}$, which is quite close to the sum of the measured barrier heights for electron and holes on our gated Au/Si SOI sample $(1.20 \mathrm{eV})$ measured at the same temperature, even though the individual barrier heights differ by more than $200 \mathrm{meV}$ from their $\mathrm{Au} / \mathrm{Si}$ counterparts. On a second gated $\mathrm{Cu} / \mathrm{Si}$ sample, the measured intrinsic barrier heights at $80 \mathrm{~K}(0.646(2) \mathrm{eV}$ for electrons and 0.558(10) eV for holes respectively) varied somewhat from the first $\mathrm{Cu} / \mathrm{Si}$ sample, but 
their sum $(1.204(5) \mathrm{eV})$ is again quite close to that of the $\mathrm{Au} / \mathrm{Si}$ and other $\mathrm{Cu} / \mathrm{Si}$ samples. This demonstrates that the large variations in individual electron- and holeSchottky barrier heights are due to variations in the local surface dipole at the $\mathrm{Au} / \mathrm{Si}$ or $\mathrm{Cu} / \mathrm{Si}$ contacts, and not due to tip artifacts or variations in local composition (such as a thin oxide layer on the $\mathrm{Si}$ ), which would have the effect of increasing the sum.

\subsection{Explanation of the applied gate voltages for $\mathrm{Cu} / \mathrm{SOI}$ and} Au/SOI

We notice that the gate voltages for BEEM and BHEM measurements on $\mathrm{Au} / \mathrm{SOI}$ and $\mathrm{Cu} / \mathrm{SOI}$ are different: for $\mathrm{Au} / \mathrm{SOI}$ they are $+7 \mathrm{~V}$ for BEEM and $-17 \mathrm{~V}$ for BHEM and for $\mathrm{Cu} / \mathrm{SOI}$ they are $+12 \mathrm{~V}$ for BEEM and $-14 \mathrm{~V}$ for BHEM. We believe this difference comes from the interface state or fixed charge at the $\mathrm{Si} / \mathrm{SiO}_{2}$ interfaces.

First, we believe that most of the applied gate voltage is across the $\mathrm{SiO}_{2}$ layer, since the voltage drop across the thin top Si film or the substrate can't be much larger than the Si bandgap. This follows by assuming that the top $\mathrm{Si}$ film is in equilibrium with the front-side ohmic contact and that the substrate is in equilibrium with the back-side ohmic contact. In this case free carrier accumulation or strong inversion at the $\mathrm{SOI}$ film $/ \mathrm{SiO}_{2}$ and $\mathrm{SiO}_{2} / \mathrm{Si}$ substrate interfaces will limit the band bending in the Si film or Si substrate to be not much larger than the Si bandgap. For a $\mathrm{p}$-Si with a doping of $\sim 10^{15} / \mathrm{cm}^{3}, \mathrm{E}_{\mathrm{F}}-\mathrm{E}_{\mathrm{V}} \approx 0.05 \mathrm{eV}$ and $\mathrm{E}_{\mathrm{C}}-\mathrm{E}_{\mathrm{F}} \approx 1.12 \mathrm{eV}$ at $\mathrm{T}=80 \mathrm{~K}$ [6], so we think the bending in the substrate will not be much larger than $\sim 0.1 \mathrm{~V}$ for 
BEEM (with a positive substrate bias) and $~ 1.1 \mathrm{~V}$ for BHEM (with a negative substrate bias).

Using Gauss's law and assuming no interface state or fixed charge at the $\mathrm{Si}$ $/ \mathrm{SiO}_{2}$ interfaces or in the $\mathrm{SiO}_{2}$, and also assuming the E-field inside the SOI film is equal to $E=\phi_{n(p) 0} / d$, we can estimate how large the gate voltage is. For measurements on $\mathrm{Au} / \mathrm{SOI}$, the estimated gate bias is $\sim+13 \mathrm{~V}$ for BEEM and $\sim-8 \mathrm{~V}$ for BHEM, which are much different from the applied gate voltage in our measurements $(+7 \mathrm{~V}$ for BEEM and $-17 \mathrm{~V}$ for $\mathrm{BHEM})$. And in $\mathrm{Cu} / \mathrm{SOI}$ case, the estimated gate voltages are $\sim+9 \mathrm{~V}$ for BEEM and $-10 \mathrm{~V}$ for BHEM.

The gate voltages used in our experiment could be explained by assuming there is interface charge (due to interfaces states) in the order of $\sim 10^{12} \mathrm{q} / \mathrm{cm}^{2}$ at $\mathrm{Si} / \mathrm{SiO}_{2}$ interfaces, in combination with some fixed charge at $\mathrm{Si} / \mathrm{SiO}_{2}$ interfaces and/or in the $\mathrm{SiO}_{2}$ bulk. Previous studies have reported the existence in SIMOX wafers of "acceptor-like" interface states above $E_{\mathrm{i}}$ (the intrinsic level in $\mathrm{Si}$ ) and "donor-like" interface states below $E_{\mathrm{i}}$ with a density in the order of $D_{i t}=10^{11}-10^{12} / \mathrm{cm}^{2} / \mathrm{eV}$, and fixed charge in the order of $D_{f}=10^{11}-10^{12} \mathrm{q} / \mathrm{cm}^{2}$, and these numbers may be larger for SIMOX wafers with thinner Si film [18,70]. To estimate the density of interface states and fix charge in our SOI sample, we assume at $\mathrm{Si} / \mathrm{SiO}_{2}$ interfaces the density of interface states is constant across the silicon band gap and the fix charge is at the $\mathrm{Si} / \mathrm{SiO} 2$ interfaces and there is no charge inside the BOX. Then using Gauss's law we can get 


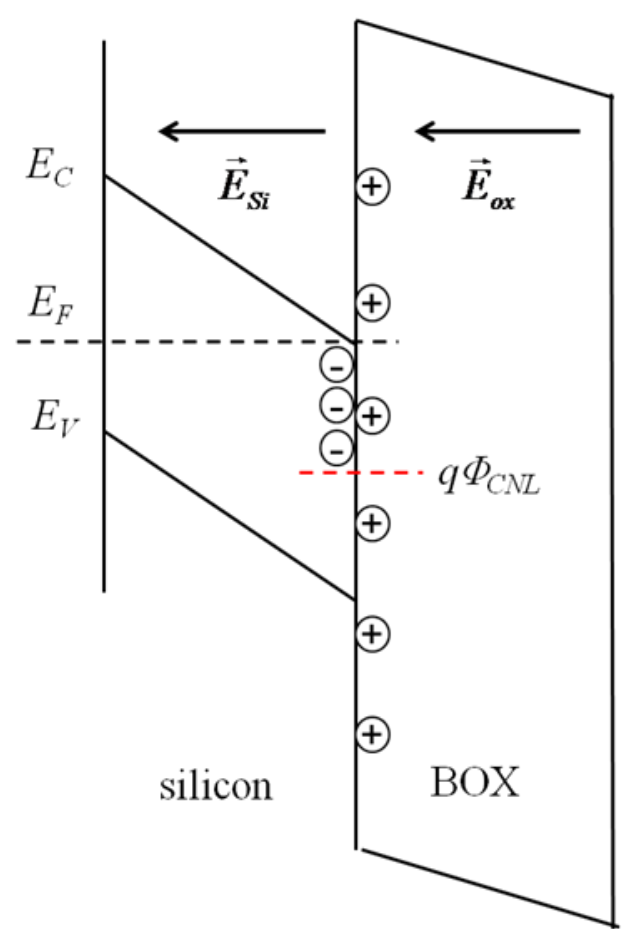

(a)

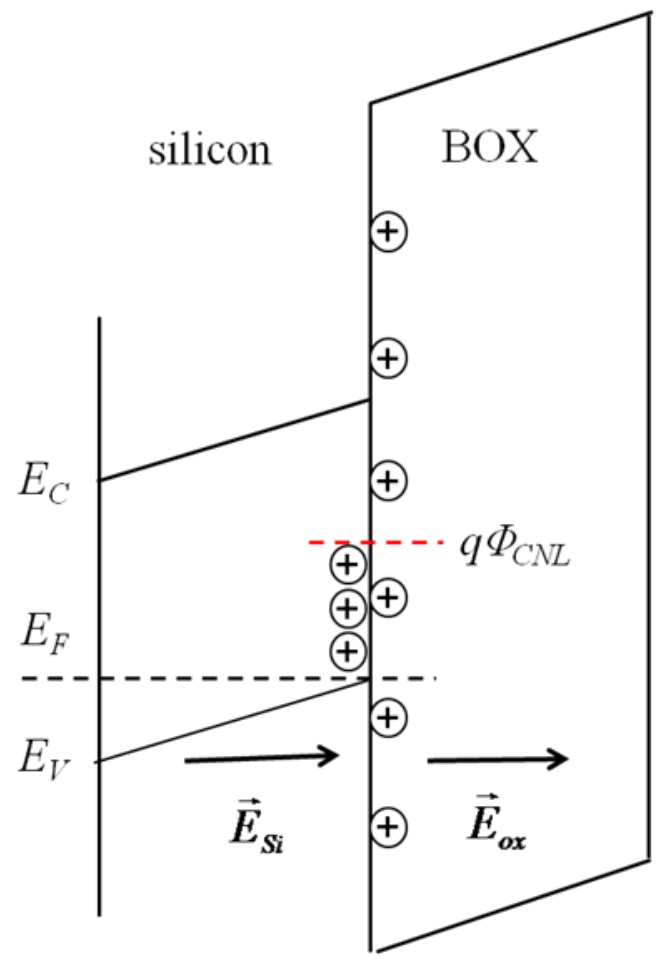

(b)

Figure 6.6: Energy band diagram for (a) positive substrate bias and (b) negative substrate bias.

$\varepsilon_{S i} E_{S i}-\varepsilon_{o x} E_{o x}=D_{f}-q D_{i t} E_{g} / 2$

for BEEM measurements and 


$$
\varepsilon_{o x} E_{o x}-\varepsilon_{S i} E_{S i}=D_{f}+q D_{i t} E_{g} / 2
$$

for BHEM measurements (Figure 6.6). In Equations (6.5), $\varepsilon_{\mathrm{Si}}=11.9 \varepsilon_{0}\left(\varepsilon_{\mathrm{ox}}=3.9 \varepsilon_{0}\right)$ is the dielectric constant of silicon (silicon oxide), and $E_{S i}\left(E_{o x}\right)$ is the E-field in the silicon (oxide) near the interface. Since we assume the conduction band minimum (valence band maximum) and the Fermi level coincide at the SOI/BOX interface for BEEM (BHEM) measurements, the interface charge is $-q D_{i t} E_{g} / 2\left(+q D_{i t} E_{g} / 2\right)$ for BEEM (BHEM) measurements. The E-field inside the silicon film can be estimated by $E_{S i}=\phi_{n(p) 0} / d$, and the electric field inside the BOX can be estimated by $E_{o x}=\left(\right.$ gate voltage $\left.-E_{g} / q\right) / d_{o x}$ where $d_{o x}=150 \mathrm{~nm}$ is the BOX thickness and it is assumed that the sum of voltage drops across the SOI and the substrate is $\sim E g / q$. In order to explain our measurements, for the Au/SOI samples we then would need to assume an average interface state density of $1.5 \times 10^{12} / \mathrm{cm}^{2} / \mathrm{eV}$ and fixed charge density of $1.2 \times 10^{12} \mathrm{q} / \mathrm{cm}^{2}$, while for the $\mathrm{Cu} / \mathrm{SOI}$ samples the interface state density would need to be $1.9 \times 10^{12} / \mathrm{cm}^{2} / \mathrm{eV}$ and the fixed charge density $0.2 \times 10^{12} \mathrm{q} / \mathrm{cm}^{2}$. These numbers agree with the numbers mentioned in Ref.[18] and Ref.[70].

We simulated the gate voltages for BEEM and BHEM measurements on our sample structure using a numerical simulation tool COMSOL Multiphysics. The details about this simulation is listed in Appendix A. Figure 6.7 is the simulation results on $\mathrm{Au} / \mathrm{SOI}$ and $\mathrm{Cu} / \mathrm{SOI}$ assuming there is no interface states at the $\mathrm{Si} / \mathrm{SiO}_{2}$ interfaces and no fix charges at the $\mathrm{Si} / \mathrm{SiO}_{2}$ or inside the $\mathrm{BOX}$, where the conduction 
band minimum and the valence band maximum are plotted relative to the Fermi level in the metal of the Schottky contacts on the SOI film. The dark blue curve is the conduction band minimum in the SOI film of $\mathrm{Au} / \mathrm{SOI}$ sample when the gate voltage is $+13 \mathrm{~V}$, and the green curve is the valence band maximum in the SOI film of $\mathrm{Au} / \mathrm{SOI}$ sample when the gate voltage is $-8 \mathrm{~V}$. The blue curve is the conduction band minimum in the SOI film of $\mathrm{Cu} / \mathrm{SOI}$ sample when the gate voltage is $+9 \mathrm{~V}$, and the red curve is

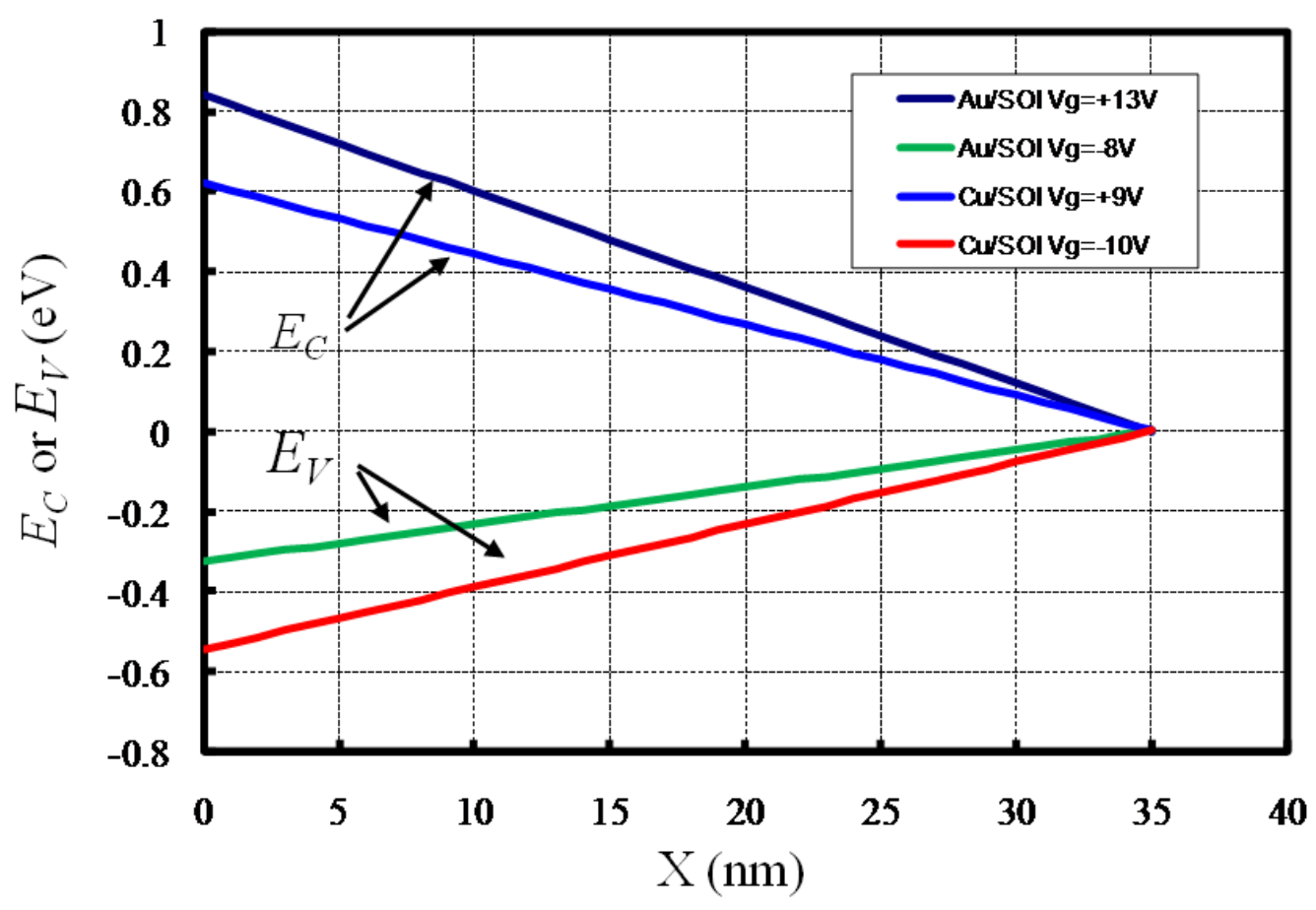

Figure 6.7: COMSOL Multiphysics simulation without interface states or fix charges. 
the valence band maximum in the SOI film of $\mathrm{Cu} / \mathrm{SOI}$ sample when the gate voltage is $-10 \mathrm{~V}$. We can see this simulation results are in good agreement with our estimation above. Figure 6.8 is the simulation results on $\mathrm{Au} / \mathrm{SOI}$ sample structure assuming there are interface states with a density of $1.5 \times 10^{12} / \mathrm{eV} / \mathrm{cm}^{2}$ and a fix charge with a density of $1.2 \times 10^{12} \mathrm{q} / \mathrm{cm}^{2}$ at the $\mathrm{Si} / \mathrm{SiO}_{2}$ interfaces. The dark blue curve is the conduction band minimum in the SOI film when the gate voltage is $+7 \mathrm{~V}$, and the red

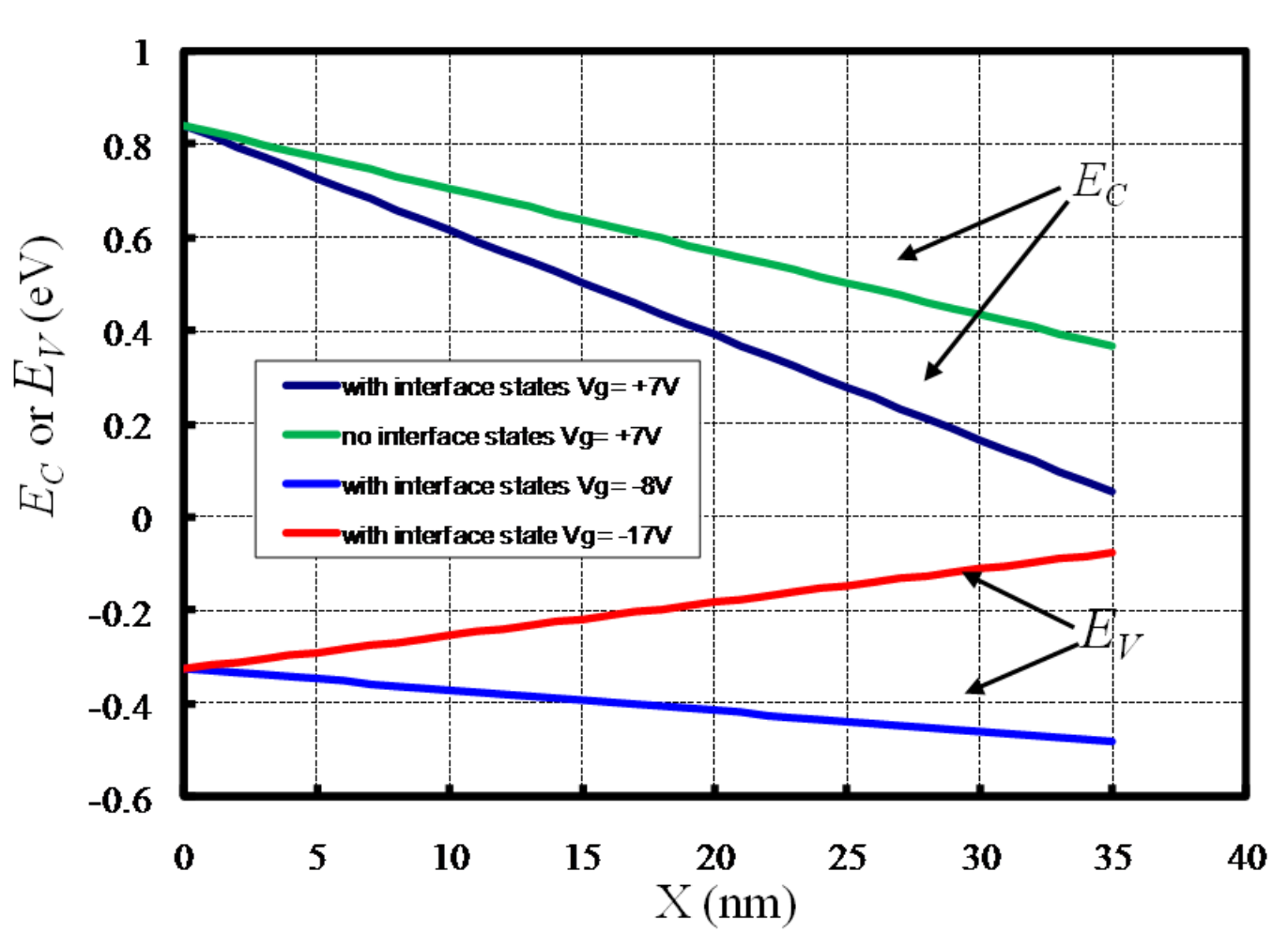

Figure 6.8: Simulation results on $\mathrm{Au} / \mathrm{SOI}$ sample structure assuming there are interface states with a density of $1.5 \times 10^{12} / \mathrm{eV} / \mathrm{cm}^{2}$ and a fix charge with a density of $1.2 \times 10^{12} q / \mathrm{cm}^{2}$ at the $\mathrm{Si} / \mathrm{SiO}_{2}$ interfaces. 
curve is the valence band maximum in the SOI film when the gate voltage is $-17 \mathrm{~V}$. For comparison, the green curve is the conduction band minimum in the SOI film when the gate voltage is $+7 \mathrm{~V}$ assuming there is no interface states or fix charge, and the blue curve is the valence band maximum in the SOI film when the gate voltage is $-8 \mathrm{~V}$. Figure 6.9 is the simulation results on $\mathrm{Cu} / \mathrm{SOI}$ sample structure assuming thereare interface states with a density of $1.9 \times 10^{12} / \mathrm{eV} / \mathrm{cm}^{2}$ and a fix charge with a

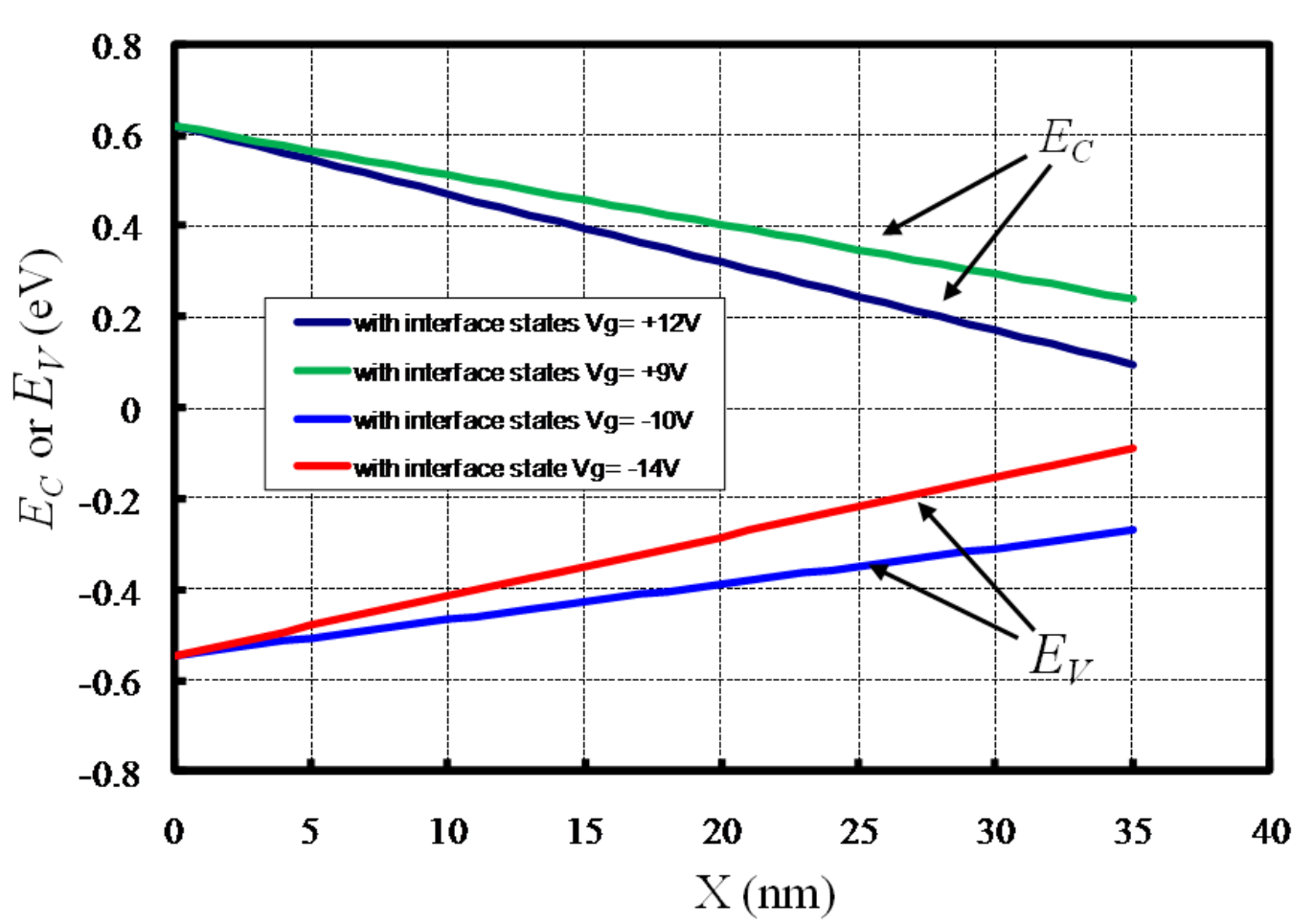

Figure 6.9: Simulation results on $\mathrm{Cu} / \mathrm{SOI}$ sample structure assuming there are interface states with a density of $1.9 \times 10^{12} / \mathrm{eV} / \mathrm{cm}^{2}$ and a fix charge with a density of $0.2 \times 10^{12} q / \mathrm{cm}^{2}$ at the $\mathrm{Si} / \mathrm{SiO}_{2}$ interfaces. 
density of $0.2 \times 10^{12} q / \mathrm{cm}^{2}$ at the $\mathrm{Si} / \mathrm{SiO}_{2}$ interfaces. The dark blue curve is the conduction band minimum in the SOI film when the gate voltage is $+12 \mathrm{~V}$, and the red curve is the valence band maximum in the SOI film when the gate voltage is $-14 \mathrm{~V}$. For comparison, the green curve is the conduction band minimum in the SOI film when the gate voltage is $+9 \mathrm{~V}$, and the blue curve is the valence band maximum in the SOI film when the gate voltage is $-10 \mathrm{~V}$. We can see adding interface states in the order of $10^{12} / \mathrm{eV} / \mathrm{cm}^{2}$ and a fix charge in the order of $10^{12} \mathrm{q} / \mathrm{cm}^{2}$ can explain the gate voltages in our BEEM and BHEM measurements qualitatively.

It is not clear why there should be a lower fixed charge density for the $\mathrm{Cu} / \mathrm{SOI}$ samples, but we do note that a much larger power was needed to thermally deposit the $\mathrm{Cu}$ film than the $\mathrm{Au}$ films (due to the lower vapor pressure of $\mathrm{Cu}$ compared to $\mathrm{Au}$ ), and that the sample likely became significantly hotter during the $\mathrm{Cu}$ deposition, which may have had an annealing effect (no intentional annealing of these samples was done). It will be nice if all of the gate voltages can be explained by assuming an interface state density and a fixed charge density with the same values. But this is really difficult or even impossible due to the following facts present in a real device. First, the interface state density is not a uniform function of energy as shown in reference [70]. Second, the alignment of the conduction or valence band relative to the Fermi energy at the $\mathrm{Si}$ film/SiO2 interface is not easy to estimate. The assumption we made in our paper is just a simple way to estimate the E field under the Schottky contacts. Third, processing can cause variations from sample to sample. 


\subsection{Image Force Lowering Measurements}

We note that for a given $V_{\text {Sch }}$ (the applied voltage between the Schottky contact and the ohmic contact to the Si film, see Figure 6.3), the measured SBH was found to be essentially independent of the substrate bias $V_{\text {sub. This is expected since }}$ varying charge accumulation at the $\mathrm{Si} / \mathrm{SiO}_{2}$ interface in response to varying $V_{\text {sub }}$ will effectively "pin" the conduction (valence) band minimum at the $\mathrm{Si} / \mathrm{SiO}_{2}$ interface to the silicon Fermi energy, causing the electrical field (and hence the Schottky barrier

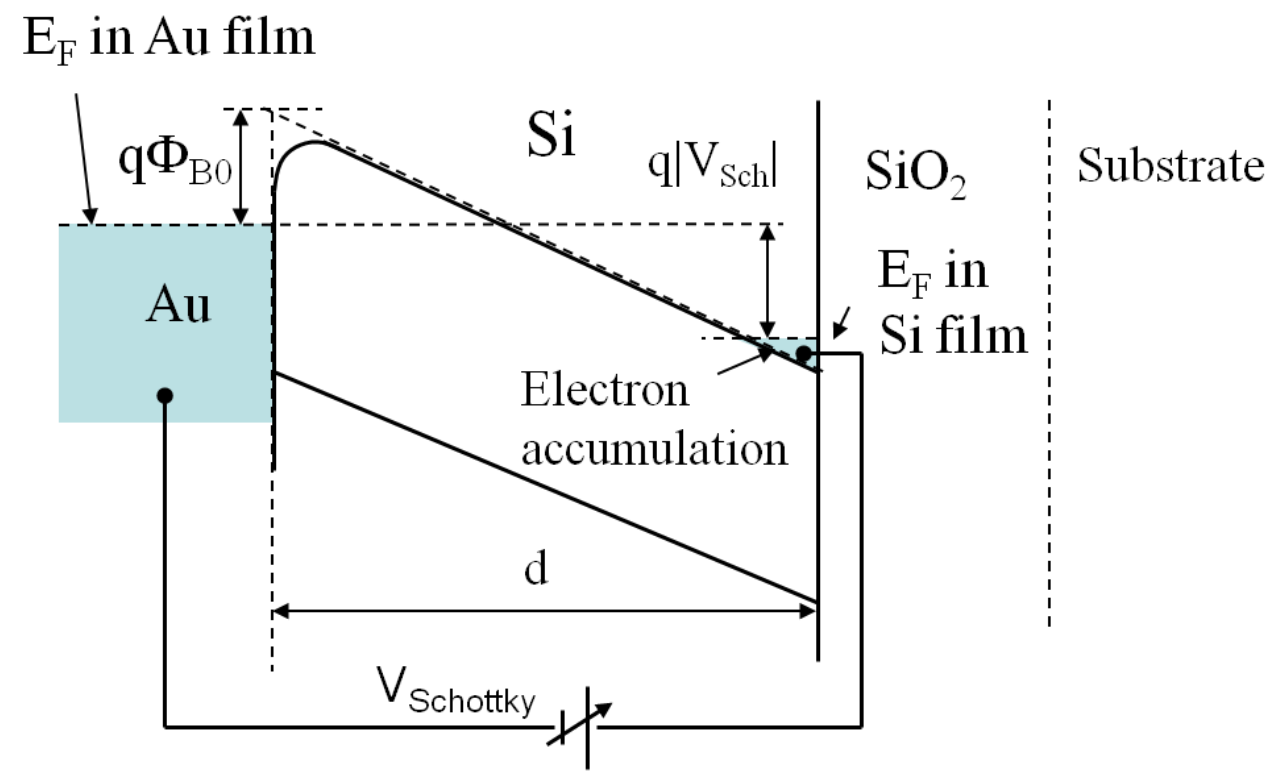

Negative bias on Schottky contact

Figure 6.10: Energy band diagrams when there is bias between the Schottky contact and the ohmic contact. 


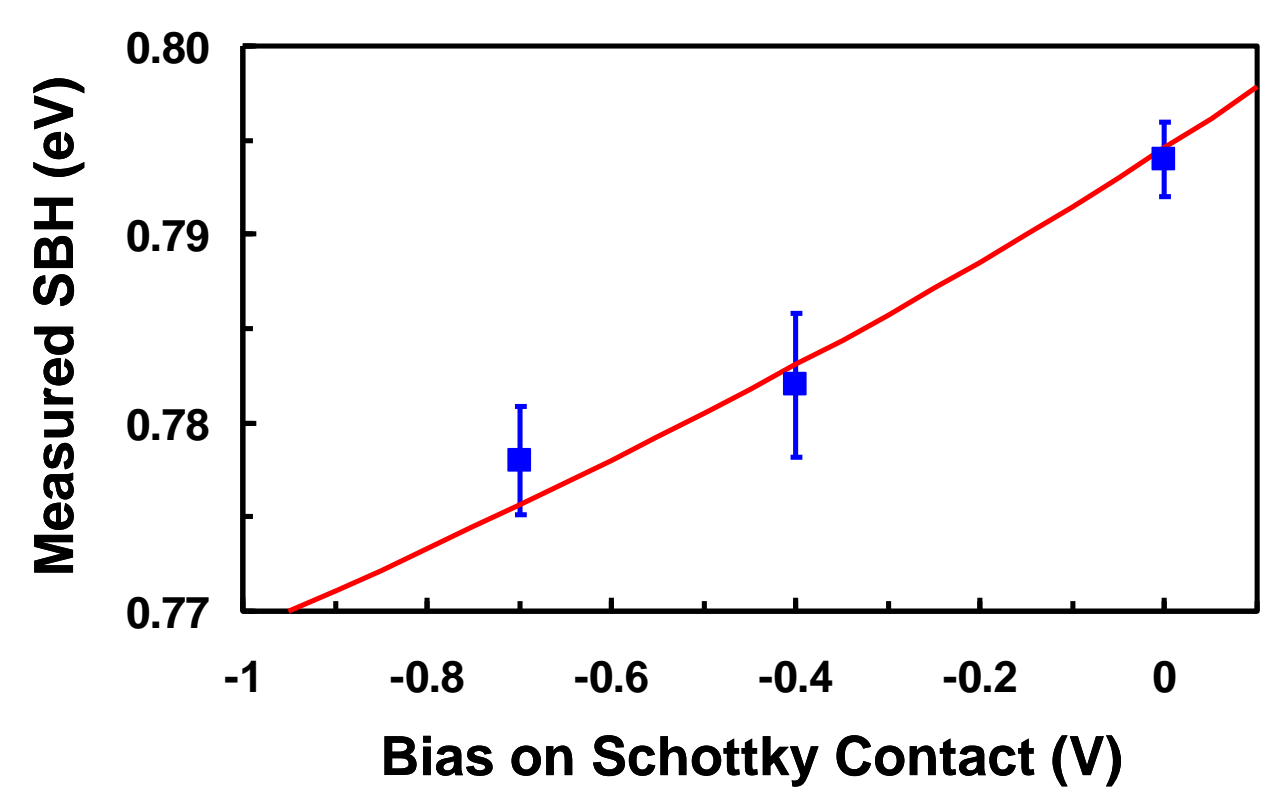

Figure 6.11: Schottky barrier heights measured on $\mathrm{Au} / \mathrm{SOI}$ samples at $\mathrm{T}=80 \mathrm{~K}$ with $\mathrm{V}_{\mathrm{Sch}}=0 \mathrm{~V}(\mathrm{SBH}=0.794(2)),-0.4 \mathrm{~V}(\mathrm{SBH}=0.782(4))$ and $-0.7 \mathrm{~V}(\mathrm{SBH}=$ $0.778(3))$.

height) to be nearly independent of $V_{\text {sub }}$, since the same electric field should produce the same image force lowering (see Section 3.3 ). All the BEEM/BHEM measurements discussed earlier in this chapter were made with $V_{\mathrm{Sch}}=0 \mathrm{~V}$. However, a non-zero value of $V_{\text {Sch }}$ should change electric field according to

$$
E=\left(V_{S c h}+\phi_{n(p) 0}\right) / d
$$


(as shown in Figure 6.10), and so should have a measurable effect on the Schottky barrier height ( see Equation 3.8 ). Figure 6.11 shows the measured electron Schottky barrier height vs $V_{\text {Sch }}$ (data points) on Au/Si SOI sample along with the predicted variation (based on Equations 6.5 and 3.8), where we see a good agreement. Therefore, we demonstrated that BEEM can monitor changes in the $\mathrm{SBH}$ due to variations in the interface electric field, and so should be able to quantify local variations in Schottky barrier heights with high spatial resolution.

\subsection{Wet etch SOI silicon film}

This work is aimed to develop a recipe for etching a $\sim 10 \mathrm{~nm}$ step on the $35 \mathrm{~nm}$ SIMOX SOI wafer. With such a recipe, we can fabricate a structure as shown in Figure 6.12. Using this structure, we can test how the Schottky barrier height can be modified by the E-field $E=\phi_{n(p) 0} / d$ by varying the film thickness $d$. And also in this structure, the electric field will be larger at the corner (marked by the red circles) than other places due to the "lightning rod effect" [26], so a lower Schottky barrier height should be seen at these corners because of image force lowering.

Since the SIMOX SOI only has a $35 \mathrm{~nm}$ silicon film, the ideal recipe should give a very slow etch rate so that it can be precisely controlled. And after etching the silicon surface should be very smooth in order to have good tip-metal tunneling characteristics in order to see BEEM signals. It has been demonstrated by Tang et al. that the standard clean 1 (SC-1, see Section 5.2) can be modified to etch the silicon 


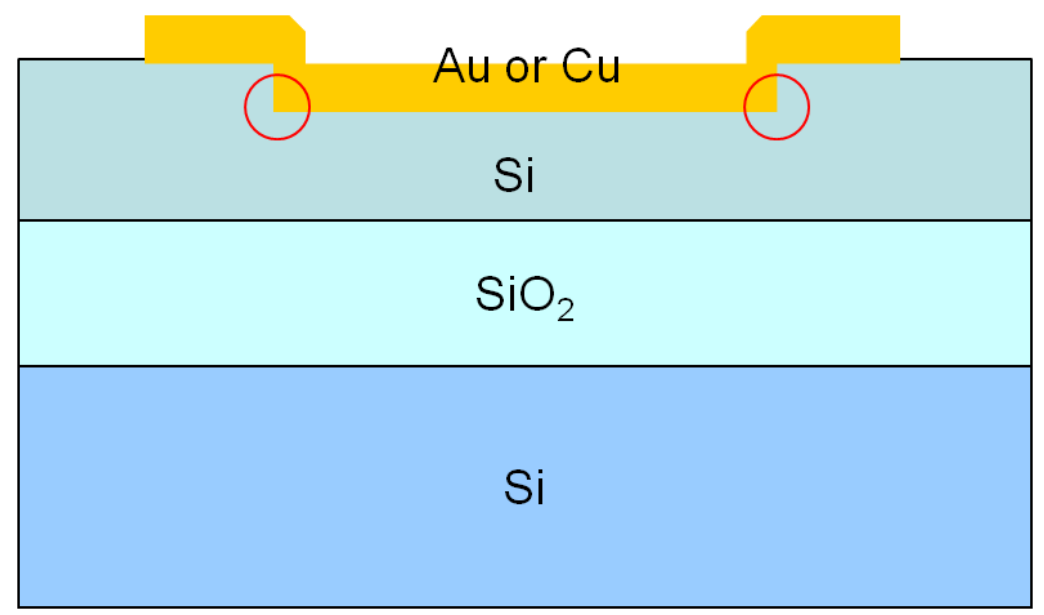

Figure 6.12: SOI sample structure with sharp corners.

film on a SIMOX wafer slowly and smoothly [71]. Their recipe is:

Solution: $\mathrm{NH}_{4} \mathrm{OH}: \mathrm{H}_{2} \mathrm{O}_{2}: \mathrm{H}_{2} \mathrm{O}=1: 8: 64$;

Temperature: $70^{\circ} \mathrm{C}$.

The etch rate they measured was $0.12 \AA / s$ and the roughness of the silicon film after etching was $4-5 \AA$.

We tried this recipe and found that for our laboratory conditions and for our SIMOX wafers the etch rate was about $0.01 \AA / s$, which is about 10 times smaller than reported by Tang et al. To increase the etch rate, we modified this recipe by 
increasing the solution density and the ratio of $\mathrm{NH}_{4} \mathrm{OH}: \mathrm{H}_{2} \mathrm{O}_{2}$. The recipe which we found to be good for us is:

Solution: $\mathrm{NH}_{4} \mathrm{OH}: \mathrm{H}_{2} \mathrm{O}_{2}: \mathrm{H}_{2} \mathrm{O}=1: 2: 16$;

Temperature: $70{ }^{\circ} \mathrm{C}$.

For our $35 \mathrm{~nm}$ SIMOX SOI, with a 15 -minute etching in a mixture of $20 \mathrm{ml} \mathrm{NH}_{4} \mathrm{OH}+$ $40 \mathrm{ml} \mathrm{H}_{2} \mathrm{O}_{2}+320 \mathrm{ml} \mathrm{H}_{2} \mathrm{O}$ at $70{ }^{\circ} \mathrm{C}$, about $10 \mathrm{~nm}$ SOI film was etched away. The RMS roughness of the silicon film after this etching was measured to be 2-3 $\AA$ using AFM ( Atomic Force Microscopy), which is similar to the roughness of Si film before the etch (see Fig. 6.13). So we see this wet etching method leaves an extremely smooth surface.

In order to etch a step as shown in Figure 6.12, we have to find a mask material that will not be etched. We found that photoresist S1813 does not work because it starts to pearl off after 10-minute dipping in our SC-1 solution. Since the etch rate of thermal oxide using the $\mathrm{SC}-1$ etch is about $\sim 5$ times slower than the etch rate of silicon [18,71], we decided to use a thermally grown thin oxide film (4-5nm) as a mask. This thin oxide film was grown by Paul Steven in Nanotech West. The growth condition was

Dry thermal oxidation at $850^{\circ} \mathrm{C}$ for 25 minutes. 

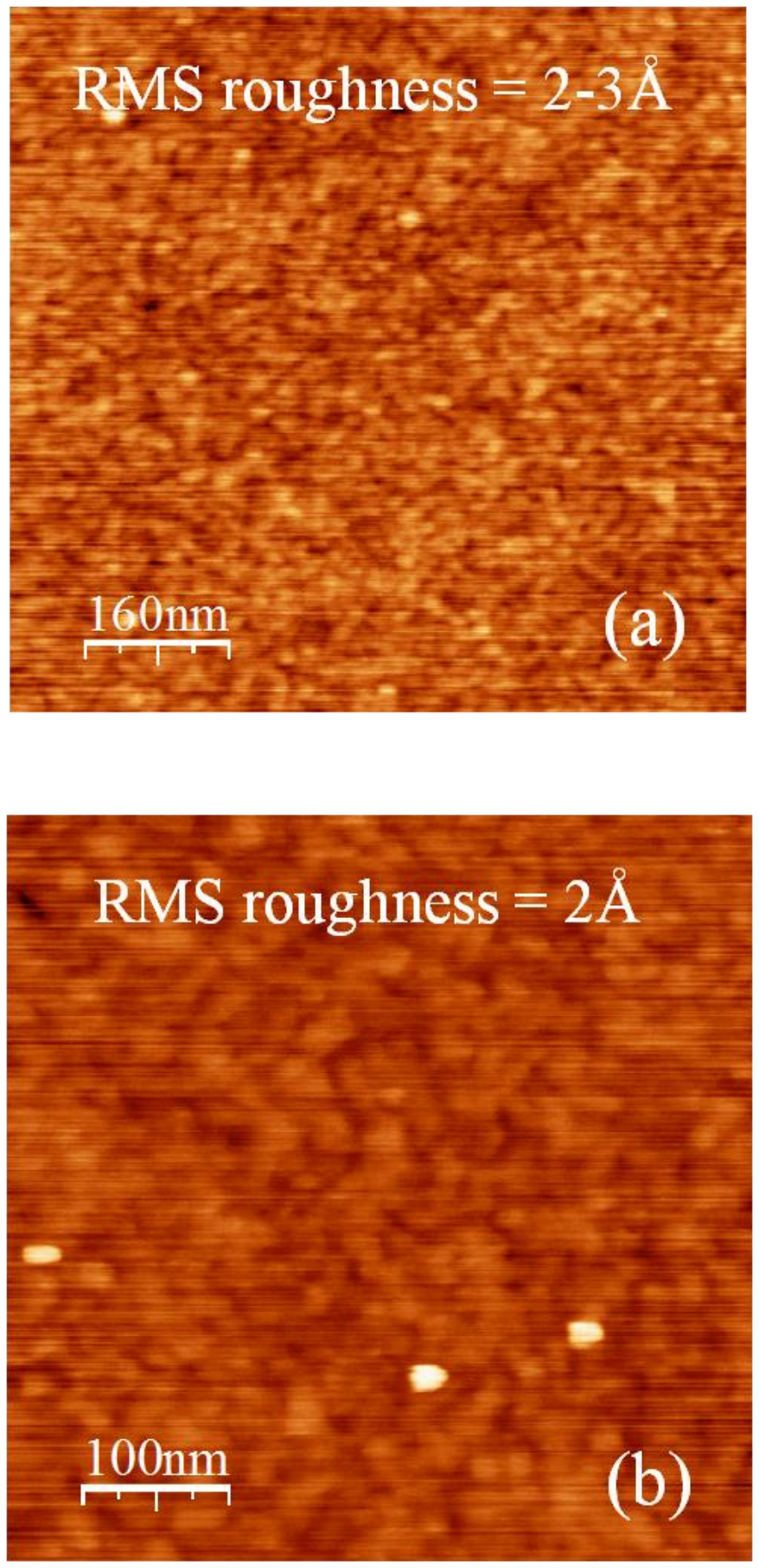

Figure 6.13: AFM images of the silicon surface (a) before and (b) after SC-1 etching. 

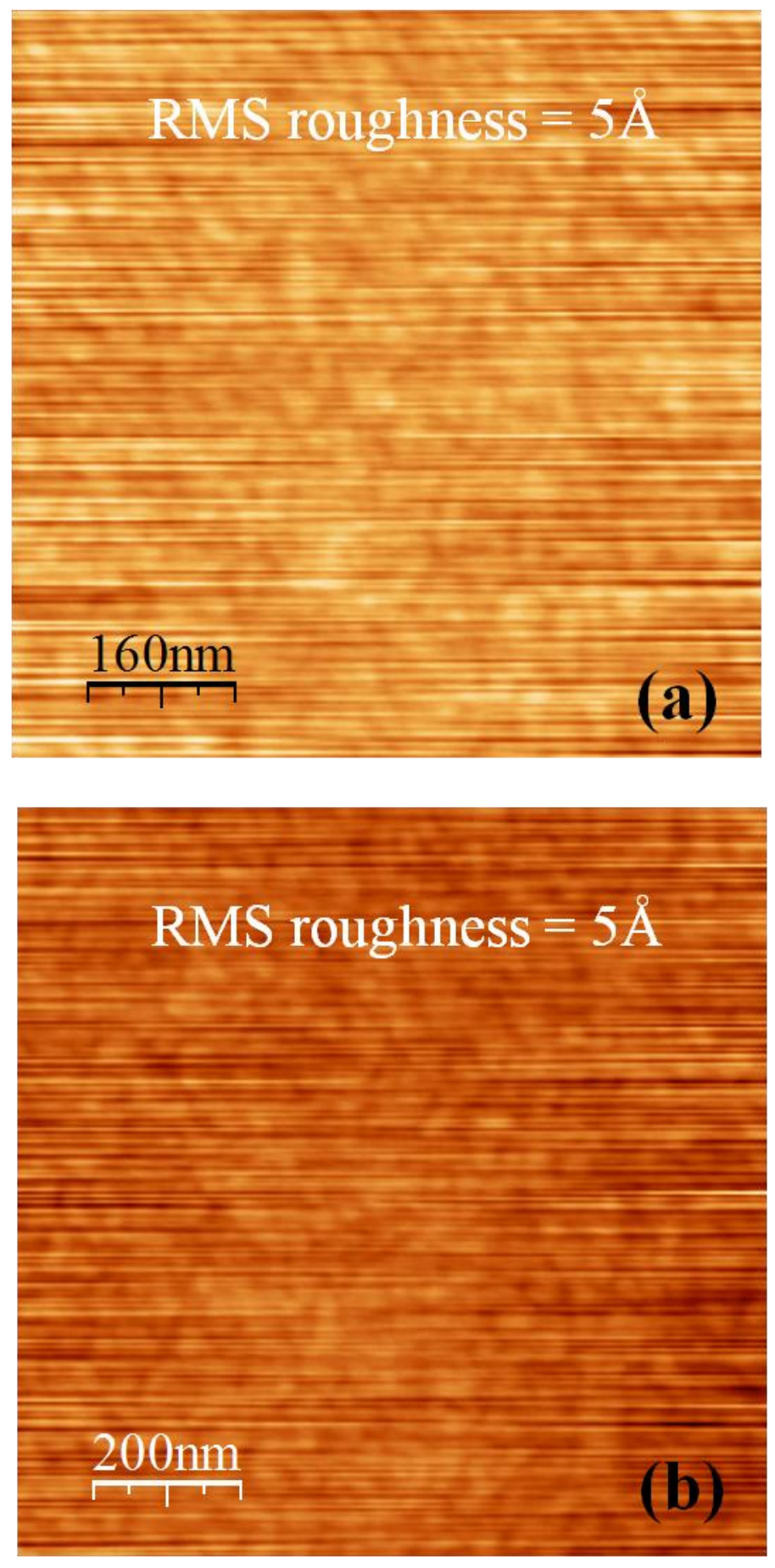

Figure 6.14: AFM images of the silicon surface after SC-1 etching (a) covered and (b) not covered by the thermal oxide. 

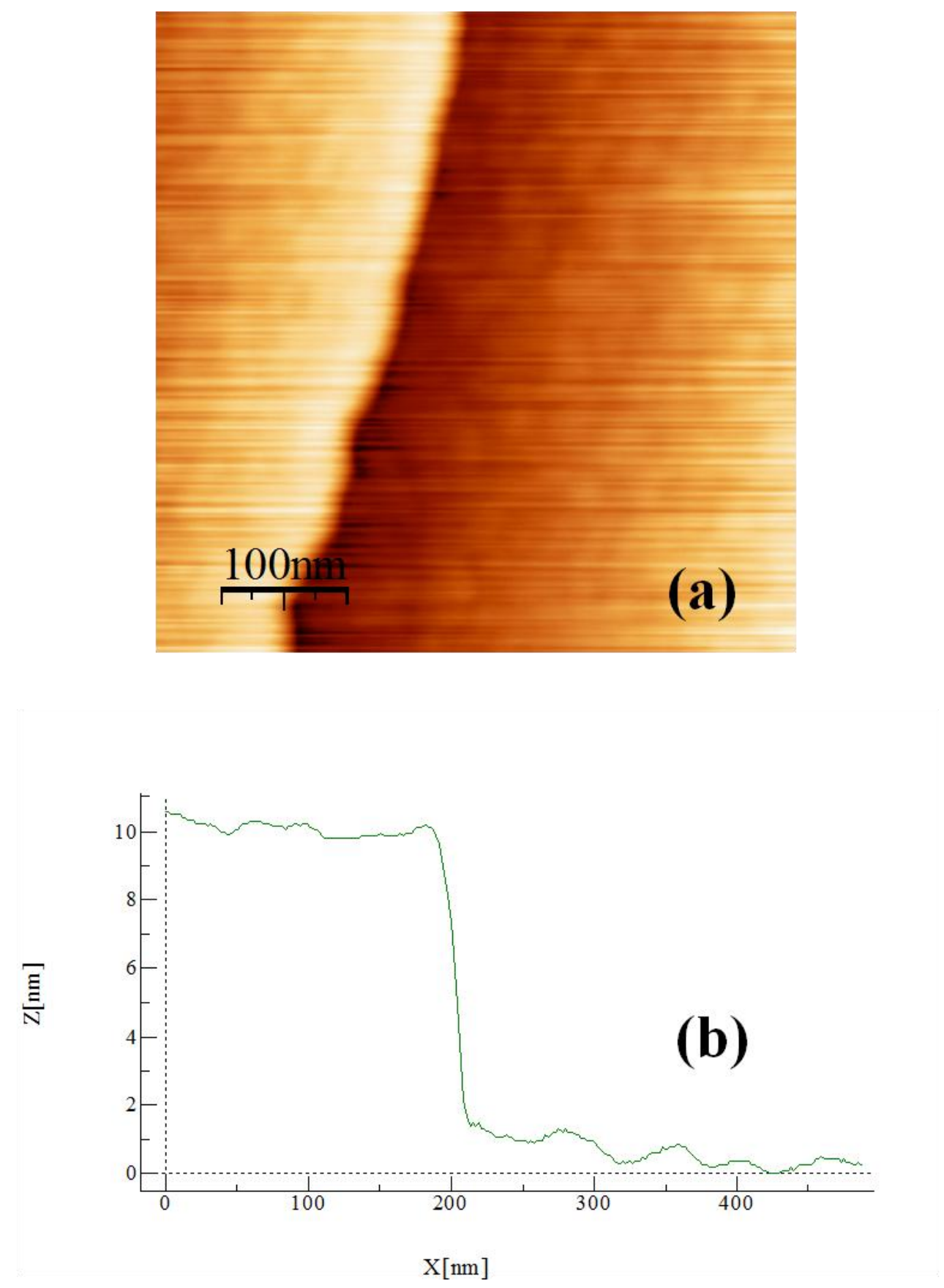

Figure 6.15. Step on the sample etched using our SC-1 recipe with $4-5 \mathrm{~nm}$ thermal oxide as a mask. 
The SIMOX SOI wafer was cleaned using Piranha cleaning (see Section 5.2 ) and then after a 10-20s 10:1 buffered HF dip the wafer was transferred to the oxide furnace for oxide film growth. After a 4-5 $\mathrm{nm}$ oxide film was grown, photolithography with S1813 was used to define areas where this thin oxide film was removed by a 10:1 buffered HF dip. Then photoresist S1813 was removed by an ultrasonic clean in Acetone and the sample was etched using our SC-1 recipe for 15 minutes. After the etching, the sample was dipped in 10:1 buffered HF for 20-30 seconds to remove all the oxide film. Figure 6.14 shows two AFM images taken on one of our SC-1 etched SIMOX SOI sample with 4-5nm thermal oxide as a mask. Figure 6.14 (a) is the AFM image taken on the area which is covered by the thermal oxide during SC-1 etch and Figure 6.14 (b) is the AFM image taken on the area which is not covered by the thermal oxide during etching. We can see the roughness ( $5 \AA$ ) is a little bigger than the roughness $(2-3 \AA)$ on samples etched without oxide growth, but it is still small and the surface after etching is very smooth. Figure 6.15 is the AFM image across the step, from which we can see the step height is $~ 9-10 \mathrm{~nm}$.

\subsection{Conclusions and future works}

In summary, we developed a new method to monitor the energy (relative to the metal Fermi energy) of both the conduction band minimum and the valence band maximum of a thin semiconductor film at the same location. By comparing these two energies, this technique can be used to monitor and quantify lateral variations of (i) the local interface electric field, (ii) local interface dipoles, and (iii) the local bandgap 
at metal/semiconductor contacts. In our measurements, we performed BEEM and BHEM measurements at the same location on the same $\mathrm{Au} / \mathrm{Si}$ and $\mathrm{Cu} / \mathrm{Si}$ contacts at $\mathrm{T}=80 \mathrm{~K}$. After accounting for image force lowering, the measured local Schottky barrier heights for electrons and holes sum to approximately the Si band gap, indicating that we can accurately probe both the conduction band and valence bands at the same location, and hence are sensitive to local variations in the metal/Si interface dipole and/or interface electric field. The gate voltages used in our experiment were explained by assuming there is interface charge (due to interfaces states) in the order of $\sim 10^{12} / \mathrm{eV} / \mathrm{cm}^{2}$ in combination with some fixed charge ) in the order of $\sim 10^{12} \mathrm{q} / \mathrm{cm}^{2}$ at $\mathrm{Si} / \mathrm{SiO}_{2}$ interfaces. We also demonstrated that BEEM can monitor changes in the SBH due to variations in the interface electric field, and so should be able to quantify local variations in Schottky barrier heights with high spatial resolution. By modifying an $\mathrm{SC}-1$ recipe, we found a method to etch a thin layer of silicon from our 35nm SIMOX SOI wafer that leaves a very smooth surface (RMS roughness $\approx 5 \AA)$ and with a very slow etch rate $(\approx 0.1 \AA / s)$ so it is easy to control how much silicon is etched away.

In the future, we will apply our ambipolar BEEM technique to study the energy band structure of some epitaxial layers such $\mathrm{MgO}$. A thin layer of $\mathrm{MgO}$ can be grown epitaxially on a silicon substrate and can be used as a tunneling barrier for spin injection. The energy band structure of thin $\mathrm{MgO}$ is not well known. Our method is a promising way to measure this. 


\section{CHAPTER 7}

\section{ION MILL AND RIE ETCHING OF SILICON NANOWIRES}

\subsection{Introduction}

Vertical Si nanowires have been used to fabricate some novel devices such as Si vertically integrated nanowire FETs or Surround-gate FETs [72, 73], Si nanowire photodetectors [7.4], and Si nanowire solar cell [75]. These devices were claimed to have some better performance than conventional structures. For example, FETs with a surrounding gate have been proposed and demonstrated to have excellent subthreshold behavior due to the high gate coupling efficiency and a $35 \%$ reduction of short-channel effects compared to double-gate (Fin FET) devices [76-79]. End-on metal contacts to vertical Si nanowires can also be used to study the role of Fermi level pinning in nano-scale metal-semiconductor contacts, which is one of the critical issues in nanowire or carbon nanotube FETs [80].

In this chapter, we will first give a brief introduction on the methods for fabricating vertical silicon nanowires. Then we will discuss our experiments that use 
ion milling to increase the yield of vertical nanowires on a nanowire sample in which the yield of vertical nanowires is not $100 \%$.

\subsection{Vertical Si nanowire fabrication}

Two groups of methods have been used to fabricate vertical Si nanowires: the "bottom up" approach and the "top down" approach. In the bottom up approach, Si nanowires are synthesized by one-dimensional epitaxial crystal growth using the vapor-liquid-solid (VLS) mechanism [81]. In this process, metal nanoclusters are used as catalyst and are spin-coated or deposited using photolithography/e-beam lithography on the semiconductor substrate surface. Then after the substrate is heated above the eutectic temperature, liquid droplets of metal/semiconductor alloy will form on the substrate surface. In the presence of a vapor-phase source of the semiconductor, the continuous feeding of the semiconductor reactant into the liquid droplet will supersaturate the eutectic. This leads to nucleation of the solid semiconductor at the liquid-solid interface and the nanowire grows. Figure 7.1 shows an SEM image of vertical Si nanowire array grown by Atwater's group from Caltech [82]. In their experiment, a 300nm thermal oxide was grown on Si (111) substrates and then was patterned using photolithography. The oxide within the patterned photoresist holes was removed by $\mathrm{HF}$ dip, then 500nm $\mathrm{Au}$ or $\mathrm{Cu}$ was thermal evaporated on the substrate. After lift-off of the photoresist, these patterned $\mathrm{Au}$ or $\mathrm{Cu}$

dots form an array of catalyst islands separated by a buffer oxide layer. Then these samples were annealed and $\mathrm{Si}$ nanowires were grown using $\mathrm{SiCl}_{4} / \mathrm{H}_{2}$. With this 


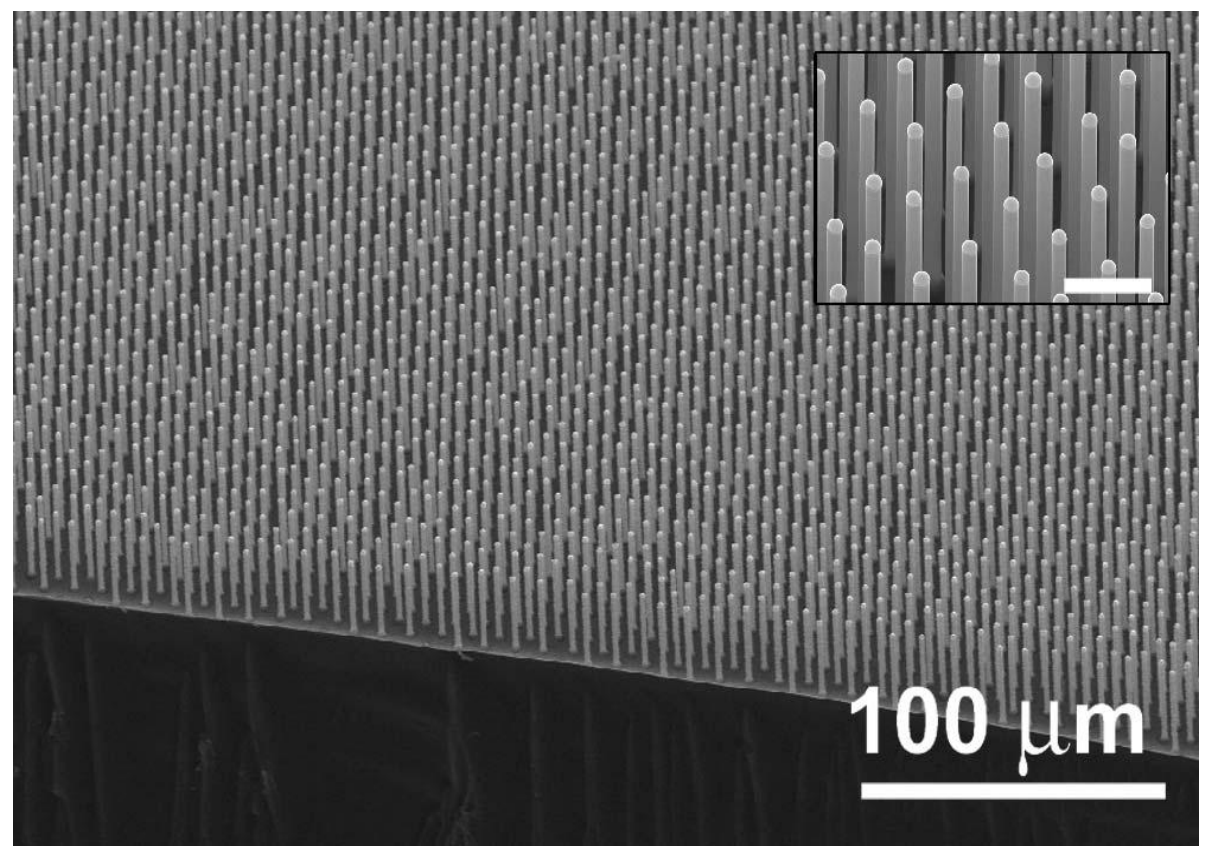

Figure 7.1: SEM views of a Cu-catalyzed Si wire array having nearly $100 \%$ fidelity over a large $1 \mathrm{~cm}^{2}$ area. The scale bar in the inset is $10 \mu \mathrm{m}$. (figure taken from Ref. [82]).

method, they attained excellent control of the size, position, and uniformity of vertically aligned, large-area Si wire arrays. The epitaxial growth on $\mathrm{Si}(111)$ substrate guarantees most of nanowires are vertical. Also Lieber et al. found that the silicon nanowire growth direction is related to the diameter for silicon nanowires grown by VLS method [83]. They demonstrated that for small silicon nanowires (diameters between 3 and $10 \mathrm{~nm}$ ) 95\% grew along the <110> direction and for large silicon nanowires (diameters between 20 and $30 \mathrm{~nm}$ ) 64\% grew along the $<111>$ direction. 


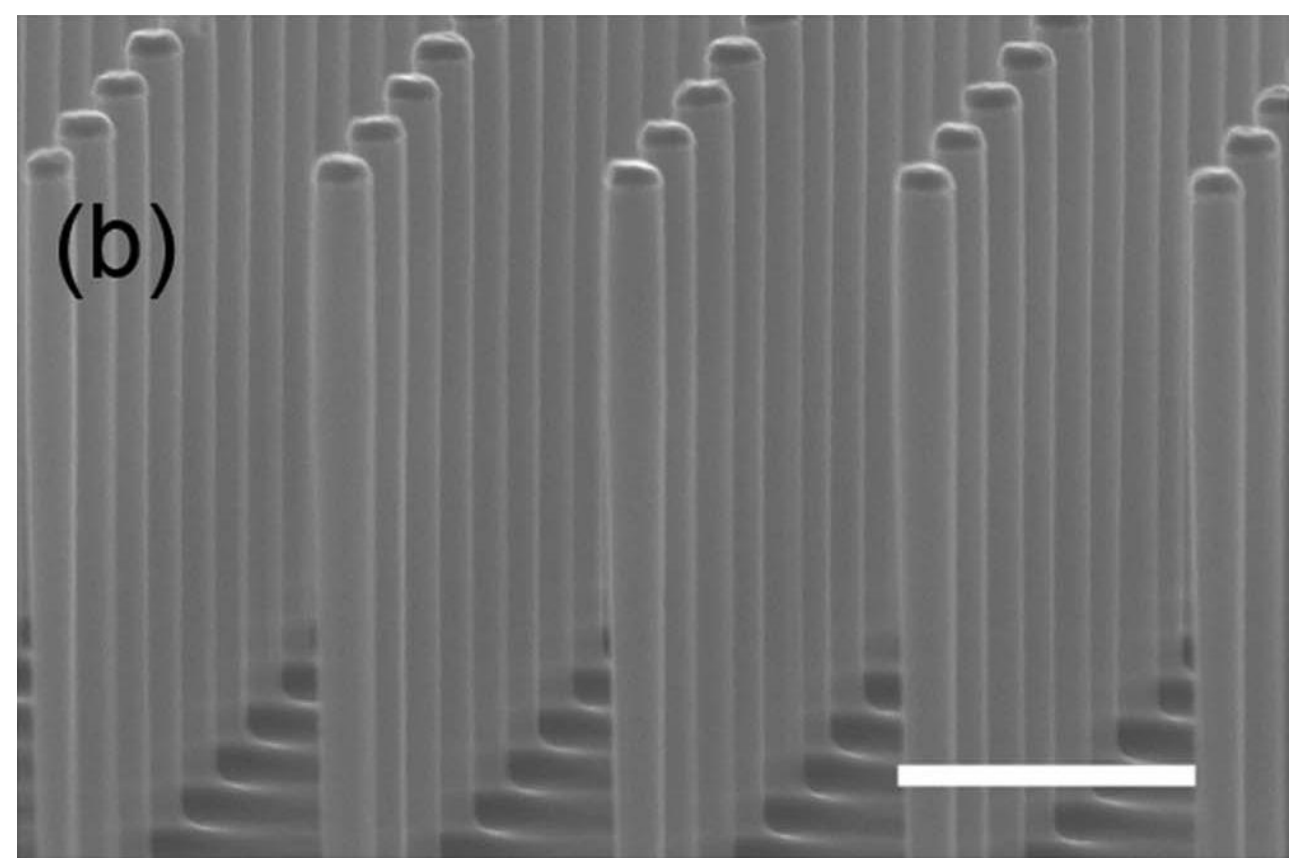

Figure 7.2: SEM of vertical-etched Si NW array device. The wires are $200 \mathrm{~nm}$ in diameter and $2.7 \mu \mathrm{m}$ in length with $1 \mu \mathrm{m}$ spacing. Scale bar is $1 \mu \mathrm{m}$. (figure taken from Ref. [74]).

In the top-down approach, vertical Si nanowires are usually fabricated using anisotropic ICP-RIE (inductively coupled plasma reactive ion etching) etching. In this process, an array of mask material with nano-scale size is patterned on Si substrates using e-beam lithography/photolithography. Then vertical $\mathrm{Si}$ nanowires can be fabricated by etching away the Si material not covered by the mask using RIE etching. The undercut and side-wall slope can be controlled by the pressure, RF bias, the composition of reactive chemicals [see the reference 84 for more details]. For example, for silicon etching using $\mathrm{SF}_{6}+\mathrm{O}_{2}$, when $\mathrm{SF}_{6} / \mathrm{O}_{2}=1$, the sidewalls are nearly 
vertical [84]. Figure 7.2 shows an SEM image of vertical Si nanowire array fabricated using ICP-RIE etching by Wang's group from UCSD [74]. In their experiment, an array of nano-scale $\mathrm{Ni}$ dots was patterned on a p-type $\mathrm{Si}$ wafer using e-beam lithography with PMMA as a resist. Then an ICP-RIE process at room temperature was used to anisotropically etch Si nanowires from the Ni dots.

\subsection{Vertical ion milling of Si nanowires}

As has been discussed in Sec.7.2, high quality vertical Si nanowires (with controllable size, position, uniformity and high fidelity) can be fabricated using both bottom-up and top-down approaches. However, finding the right recipe for growing or fabricating this kind of high quality vertical Si nanowires is not easy. In most cases the vertical Si nanwire samples we received from collaborators had Si nanowires lying on the substrate, some were tilted at an angle (but were not perpendicular to the substrate), and the rest were perfectly vertical. It seems that small changes in growth parameters (temperature, nanowire doping, substrate orientation, diameter of catalyst particles, etc.) caused many of the nanowires to grow in a non-vertical orientation.

Here we discuss a method to increase the yield of vertical Si nanowires on such "imperfect" samples using ion milling or RIE. This method may be useful to those who need to increase the fraction of vertical nanowires on samples with a high density of non-vertical nanowires.

Before discussing the details of ion milling vertical Si nanowire, we first discuss the equipment used for performing the ion milling and measuring scanning 
electron microscope (SEM) images of the samples before and after the ion milling. The equipment used for these experiments is the FEI Helios Nanolab 600 Dual Beam Focused Ion Beam/Scanning Electron Microscope [85]. This piece of equipment has both an electron beam for SEM imaging and $\mathrm{a} \mathrm{Ga}^{+}$ion beam for ion milling. The range of beam energy is $50 \mathrm{eV}-30 \mathrm{keV}$ and $500 \mathrm{eV}-30 \mathrm{keV}$ for the electron and $\mathrm{Ga}+$ beams respectively. The size of both particle beams can be adjusted by the user, and both particle beams can be raster-scanned over the sample surface. The electron beam and the ion beam form an angle of 52 degrees [Figure 7.3]. The sample stage can be rotated up to an angle of 52 degrees so that an SEM image can be taken at any angle

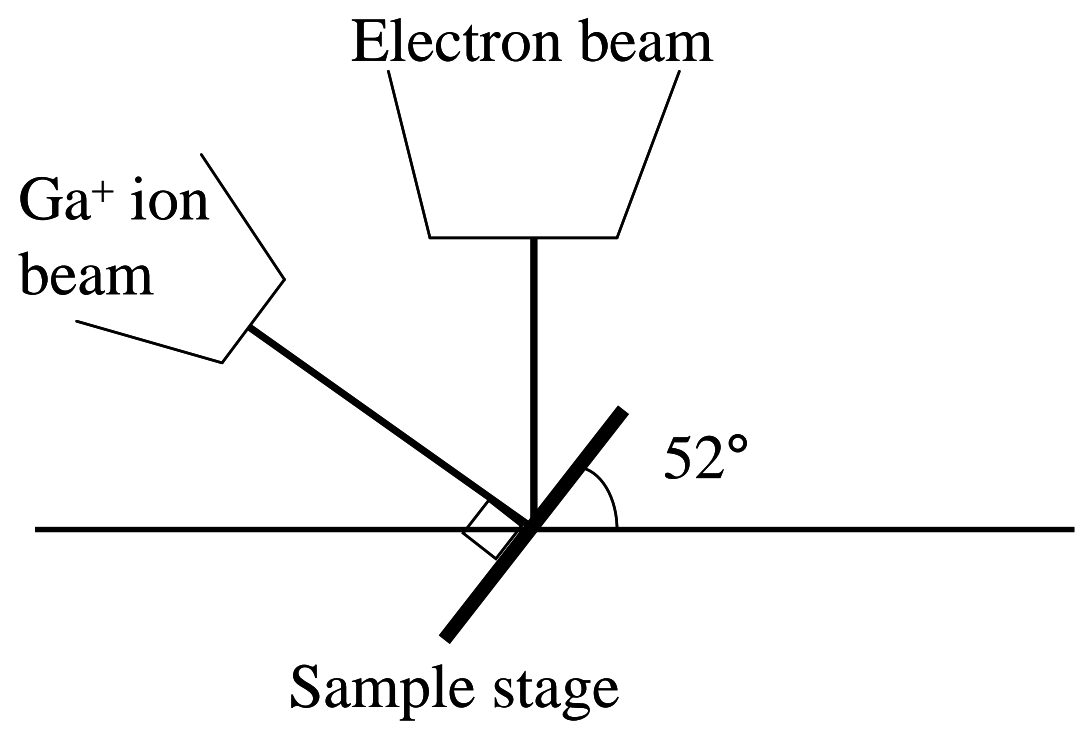

Figure 7.3: Dual Beam Focused Ion Beam/Scanning Electron Microscope. 


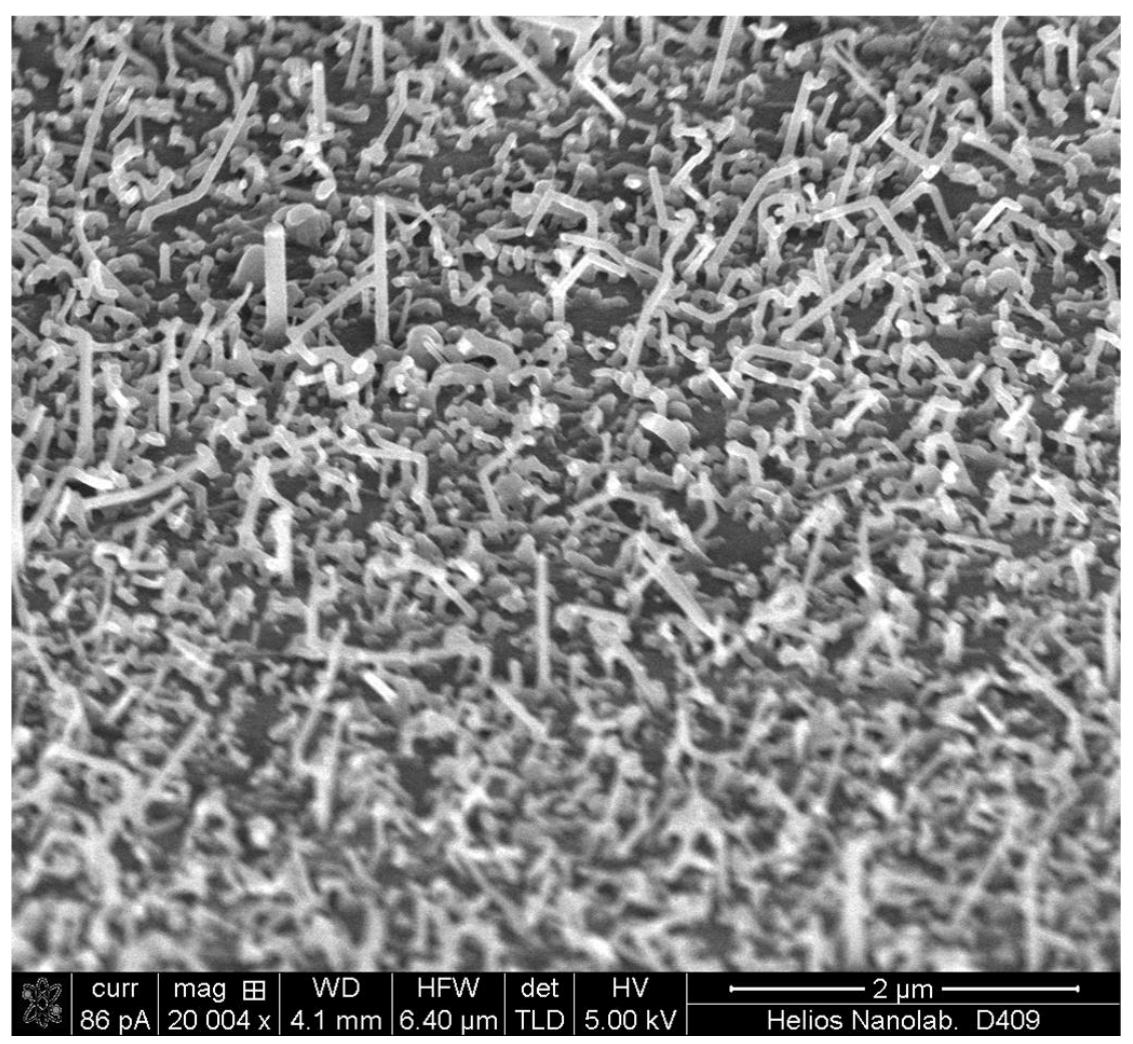

Figure 7.4: An SEM of Si nanowire sample taken at 52 degree.

between 0 to 52 degrees to the surface normal. The sample surface can be oriented perpendicular to the ion beam for "vertical" ion milling by the high energy heavy $\mathrm{Ga}^{+}$ ions.

In our experiments, the vertical $\mathrm{Si}$ nanowire samples were obtained from Lauhon's group in Northwest University [86]. These vertical Si NWs were epitaxially grown on an n-Si (111) substrate by chemical vapor deposition (CVD) at $450{ }^{\circ} \mathrm{C}$ with dispersed colloidal Au particles as catalyst and silane as Si source. Figure 7.4 shows 
an SEM image of this Si nanowire sample measured with the electron beam at $52^{\circ}$ from the surface normal. We can see from Fig. 7.4 that there are some vertical Si nanowires, but that most of the Si nanowires are lying flat on the substrate or are tilted at an angle less than $90^{\circ}$ from the surface. We found that the highly anisotropic ion milling from the focused $\mathrm{Ga}^{+}$ion beam oriented normal to the sample surface can

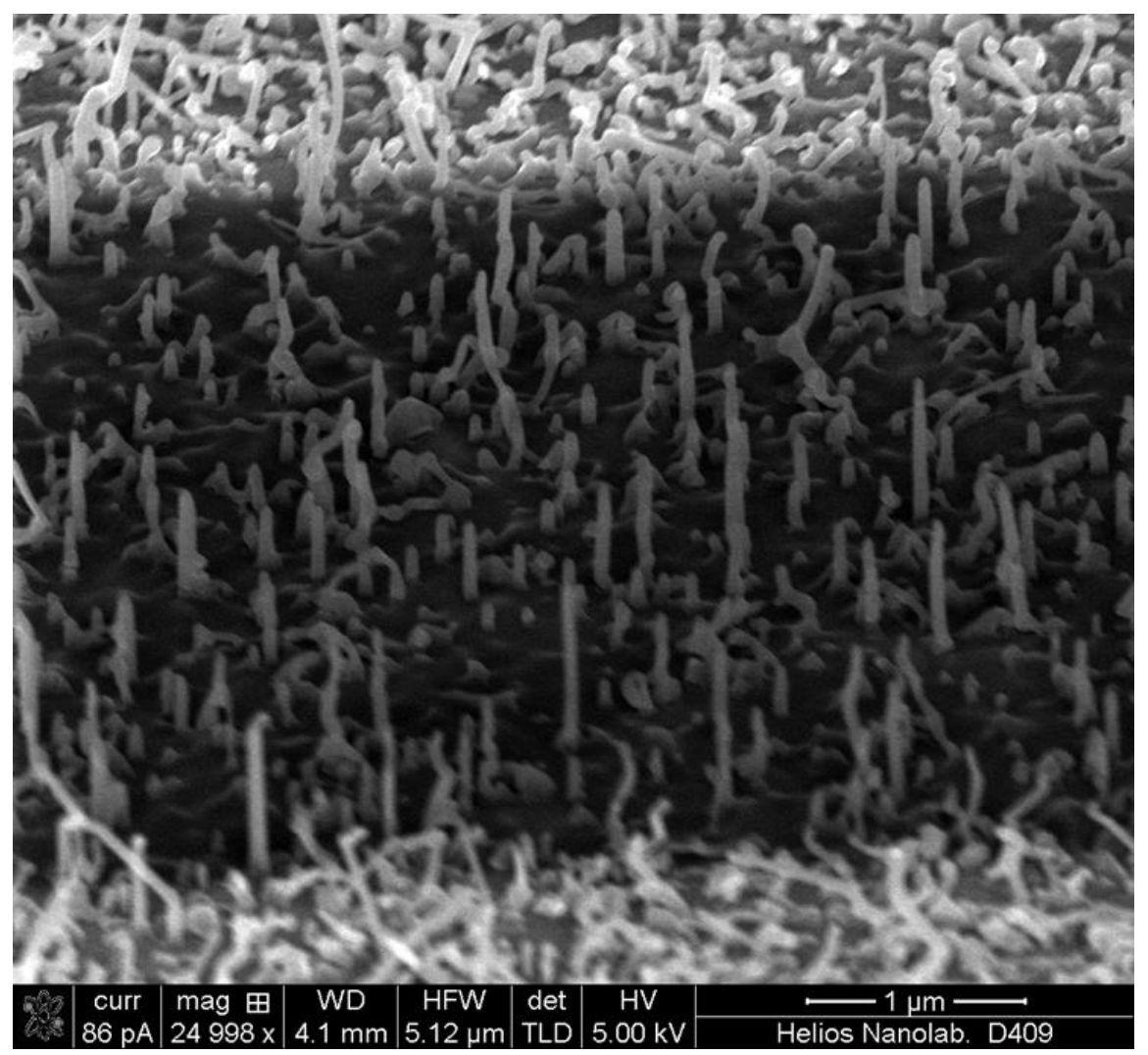

Figure 7.5: A $52^{\circ} \mathrm{SEM}$ image of Si nanowire sample after ion milling expected to etch $10 \mathrm{~nm}$ of Si. 
be a good tool to remove non-vertical Si nanowires, while leaving the vertical nanowires largely intact.

To do the ion milling, we first followed the standard operation procedure of the SEM to focus the electron beam on the sample surface and to bring the sample surface to a distance of $4.1 \mathrm{~mm}$ away from the electron gun. Then the stage was carefully rotated by $52^{\circ}$ so that the ion beam was perpendicular to the sample surface. Then ion milling over an area of $5 \mathrm{um} \times 5 \mathrm{um}$ was done by an amount that was expected to etch about $10 \mathrm{~nm}$ of $\mathrm{Si}$. Another SEM image was then taken with the sample still

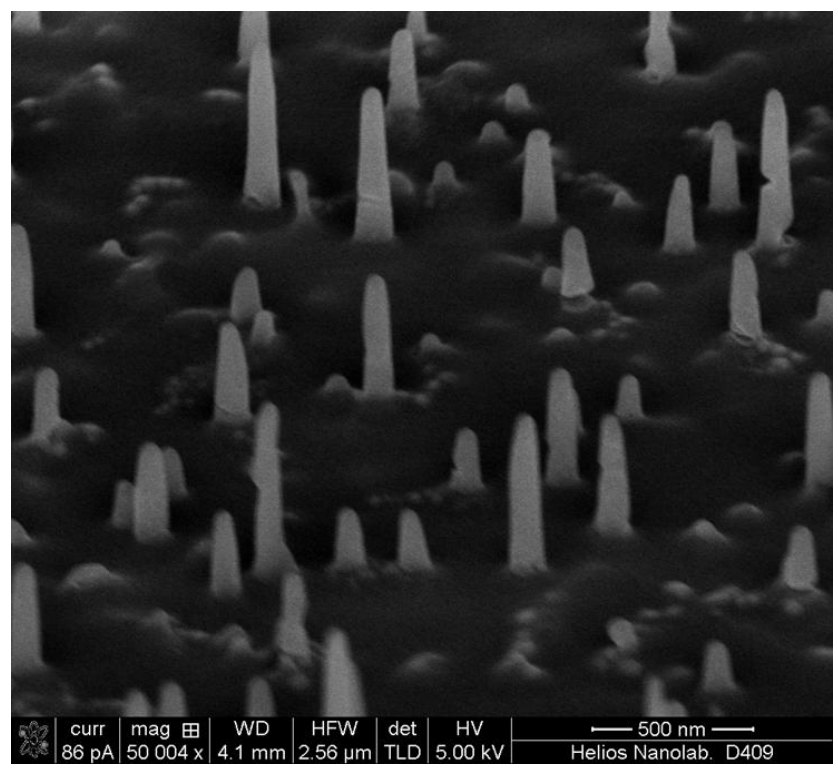

Figure 7.6: A $52^{\circ} \mathrm{SEM}$ image of Si nanowire sample after ion milling expected to etch $30 \mathrm{~nm}$ of Si. 


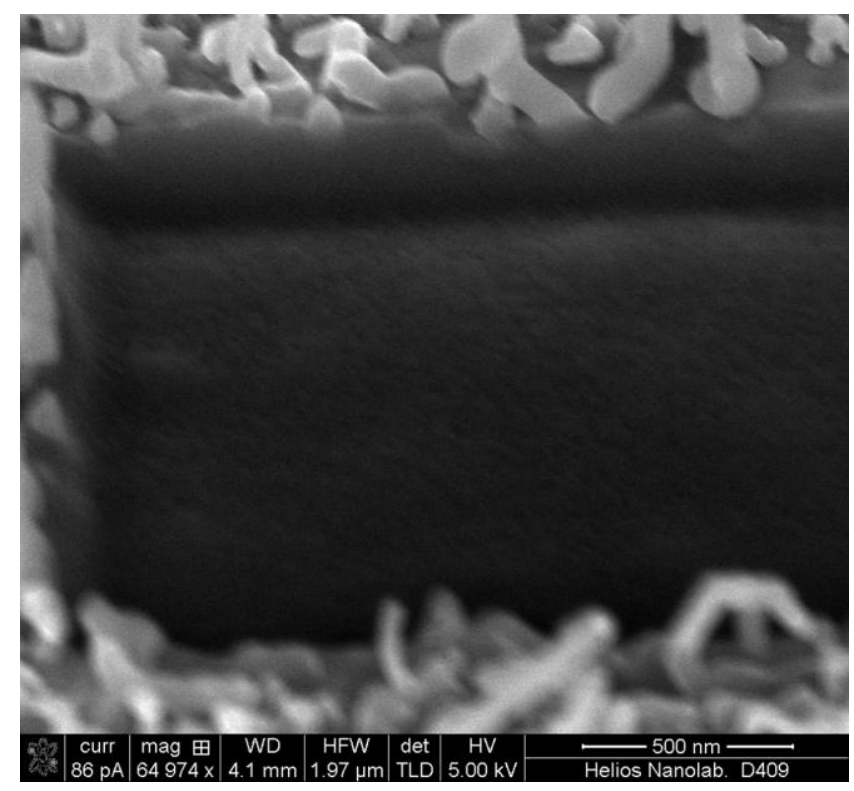

Figure 7.7: A $52^{\circ} \mathrm{SEM}$ image of $\mathrm{Si}$ nanowire sample after ion milling expected to etch $200 \mathrm{~nm}$ of $\mathrm{Si}$.

facing the ion beam, as shown in Figure 7.5. From this image we can see that most of the Si nanowires which survived from the ion milling are vertical Si nanowires, and that most of the non-vertical Si nanowries were milled away. We can also notice that some nanowires seem to be etched more than others, which could be a problem for some applications. Figure 7.6 shows an SEM image of an area after an ion milling expected to etch roughly $30 \mathrm{~nm}$ of $\mathrm{Si}$, and here we can see essentially only vertical $\mathrm{Si}$ nanowires remaining, with essentially all the non-vertical Si nanowires removed. For 
comparison, an SEM image of an area with an expected 200nm of Si ion milling is shown in Figure 7.7 from which we can see all of the Si nanowires were milled away.

\subsection{ICP-RIE etch silicon nanowires.}

We also tried to use low temperature ICP-RIE etching to increase the yield of vertical silicon nanowire in a large area, since ICP-RIE etching is a more common etching tool for Si processing than ion milling, and because it produces more uniform etching over large areas. The reactive gases we used were a mixture of $\mathrm{SF}_{6}$ and $\mathrm{O}_{2}$, which was reported to produce high aspect ratio etching of silicon because $\mathrm{O}_{2}$ in $\mathrm{SF}_{6}$ plasma can form a thin fluorinated silicon oxide passivation layer on the (vertical) sidewalls (which can prevent chemical etching of the Si on the sidewalls), while the physical etching of the ions (which are mainly moving in the vertical direction) removes the passivation layer on horizontal surfaces [84]. Cooling the substrate can also slow down the lateral etching, resulting a high aspect ratio etching.

The RIE etcher used for these experiments was the Oxford 180 ICP-RIE in the Dreese Lab cleanroom. In this system, there are two RF power sources: one is the "ICP power" which is mainly used to create the plasma, and the other is mainly used to accelerate ions in the "vertical" direction (normal to the sample surface) (Figure 7.8). The sample stage temperature can varied from $-150{ }^{\circ} \mathrm{C}$ to $+400{ }^{\circ} \mathrm{C}$ by liquid $\mathrm{N}_{2}$ cooling or electrical heating. 


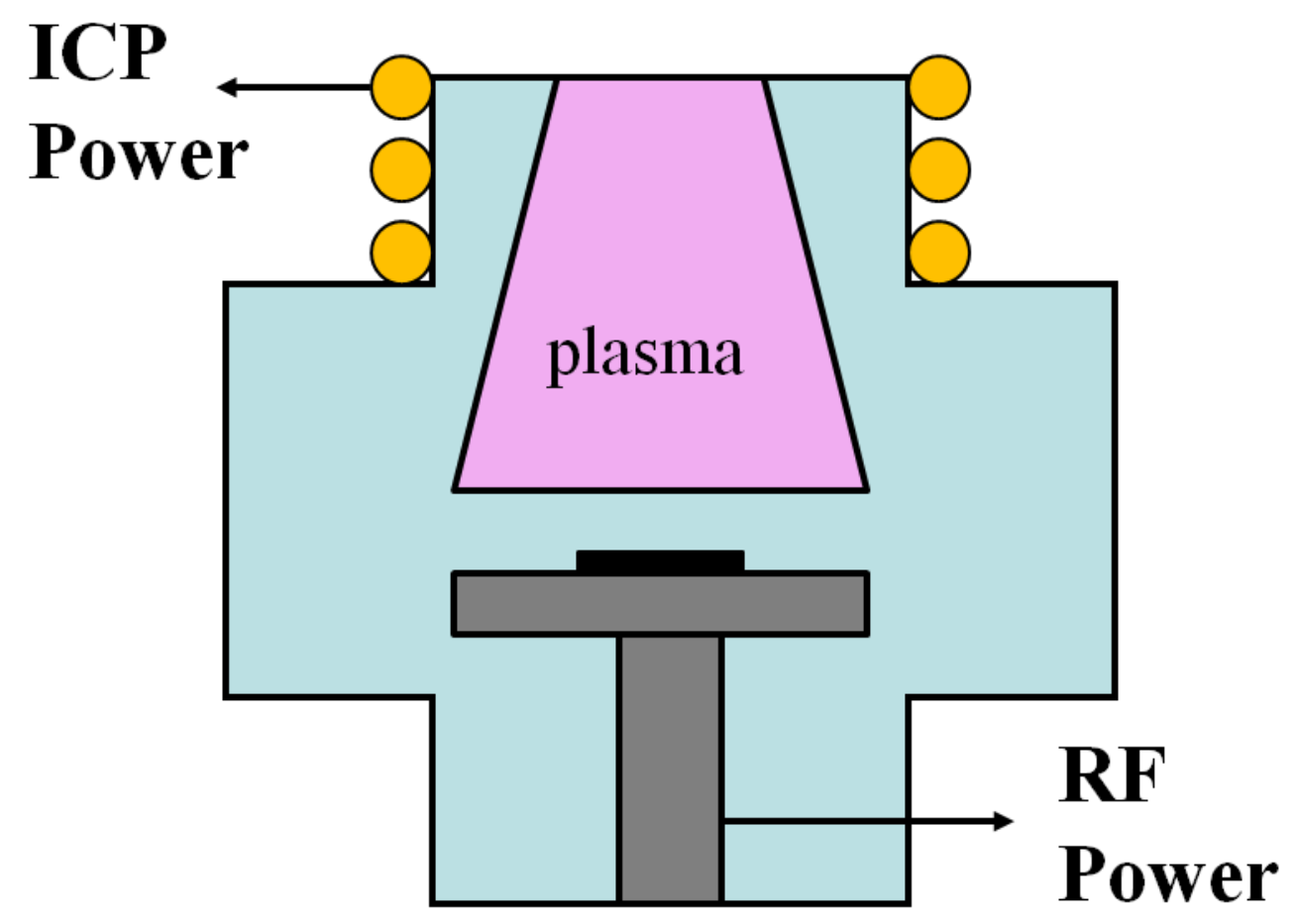

Figure 7.8: Schematic of ICP-RIE.

We found a recipe which can give nearly vertical etching of a bulk silicon sample. The recipe is

$$
\begin{aligned}
& \mathrm{SF} 6: \mathrm{O} 2=25 \mathrm{sccm}: 25 \mathrm{sccm} \\
& \text { ICP power }=500 \mathrm{~W} ; \\
& \text { RF power }=90 \mathrm{~W} ; \\
& \text { Pressure }=15 \mathrm{mT} \text { Torr; }
\end{aligned}
$$




$$
\text { Temperature }=-110^{\circ} \mathrm{C} \text {. }
$$

However, we found two problems with this recipe. First, even though the average etching is almost vertical the sidewalls after etching were found to be rough. Second, the etch rate of $\sim 18 \mathrm{~nm} / \mathrm{s}$ for bulk Si was found to be too fast, making it very hard to control the etch depth. At present we are working on finding a recipe which can give a high aspect ratio etch (sidewall angle $\left.\approx 90^{\circ}\right)$ and a slow etch rate $(<2 \mathrm{~nm} / \mathrm{s}$ ). We do not yet know if ICP-RIE etching can effectively remove non-vertical $\mathrm{Si}$ nanowires while leaving vertical Si NWs largely intact.

\subsection{Conclusions and future work}

From the above discussion, we can conclude that by choose the correct ion milling thickness of silicon essentially all of the non-vertical silicon nanowires can be removed, leaving essentially only vertical silicon nanowires standing on the substrate. While this method is not able to fabricate a highly-uniform array of vertical silicon nanowires from a highly non-uniform starting sample (such as that shown in Fig. 7.4), but may be useful as a means to remove most non-veritical nanowires if they exist. In the future, we will try to use low-temperature ICP-RIE (Inductively Coupled Plasma Reactive Ion Etching) to do the same work, since ICP-RIE can etch a larger area than Ion Milling and is a more common tool for Si processing. 


\section{APPENDIX A}

\section{NUMERICAL SIMULATION OF GATE VOLTAGES}

The gate voltages needed for our BEEM and BHEM measurements on SOI samples was simulated using a commercial numerical simulation tool COMSOL MultiPhysics 4.0 which can solve coupled differential equations using finite element methods.

Figure A.1 shows the geometry and symbols used in this simulation.

1. Parameters:

1) Bandgap $E g$ :
a. Silicon: $E g_{S i}=1.17 \mathrm{eV}$.
b. $\mathrm{SiO}_{2}: E g_{\text {ox }}=9 \mathrm{eV}$

2) Electron affinity $\chi$ :

a. Silicon: $\chi_{\mathrm{Si}}=4.05 \mathrm{eV}$ 
b. $\mathrm{SiO}_{2}: \chi_{\mathrm{ox}}=0.95 \mathrm{eV}$

3) Schottky barrier height (SBH) for electrons
a. $\mathrm{Au} / \mathrm{Si}: \mathrm{SBH}=0.84 \mathrm{eV}$
b. $\mathrm{Au} / \mathrm{Si}: \mathrm{SBH}=0.62 \mathrm{eV}$

4) Dielectric constant $\varepsilon$ :
a. Silicon: $\varepsilon_{\mathrm{Si}}=11.9 \varepsilon_{0}$.
b. $\mathrm{SiO}_{2}: \varepsilon_{\mathrm{ox}}=3.9 \varepsilon_{0}$.

5) Geometry:
a. Silicon film thickness: $d_{S i}=35 \mathrm{~nm}$.
b. BOX thickness: $d_{o x}=150 \mathrm{~nm}$.
c. Silicon subtrate thickness: $d_{s u b}=3000 \mathrm{~nm}$.

6) Doping:

$$
\begin{aligned}
& N_{A}=1.0 \times 10^{15} / \mathrm{cm}^{3} \text { in Regions } \mathrm{R} 1 \text { and } \mathrm{R} 3 \\
& N_{A}=0 \text { in Region R2. }
\end{aligned}
$$

7) Temperature: $\mathrm{T}=80 \mathrm{~K}$.

8) Boltzmann constant: $k_{B}=1.381 \times 10^{-23} \mathrm{~J} / \mathrm{K}$.

9) Elementary charge: $q=1.602 \times 10^{-19} \mathrm{C}$.

10) Permitivity in vacuum: $\varepsilon_{0}=8.854 \times 10^{-14} \mathrm{~F} / \mathrm{cm}$.

11) Intrinsic carrier density $n_{i}=6.07 \times 10^{-14} / \mathrm{cm}^{3}$ at $80 \mathrm{~K}$.

12) Charge neutrality level (CNL) $\phi_{\mathrm{CNL}}=E_{i}$. 
2. Variables:

a. Applied substrate voltage: $V_{a}(\mathrm{~V})$.

b. Interface state density at $\mathrm{Si} / \mathrm{SiO} 2$ interface: $D_{i t}\left(1 / \mathrm{cm}^{2} / \mathrm{eV}\right)$.

c. Fixed charge density at $\mathrm{Si} / \mathrm{SiO} 2$ interface: $D_{f}\left(q / \mathrm{cm}^{2}\right)$.

3. Model: AC/DC modeling $\rightarrow$ electrostatics interface.

Equation: $-\nabla \cdot(\varepsilon \nabla \psi)=\rho$

$$
\rho=q\left(p-n-N_{A}\right)
$$

a. Region R1:

$$
\begin{aligned}
& n=n_{i} \exp \left(\left(q \psi+E g_{S i} / 2-S B H 1\right) / k_{B} T\right) \\
& p=n_{i} \exp \left(\left(S B H 1-q \psi-E g_{S i} / 2\right) / k_{B} T\right)
\end{aligned}
$$

b. Region R2: $p=0, n=0, N_{A}{ }^{-}=0$.

c. Region R3:

$$
\begin{aligned}
& n=n_{i} \exp \left(\left(q \psi+E g_{S i} / 2-S B H 1-q V_{a}\right) / k_{B} T\right) \\
& p=n_{i} \exp \left(\left(S B H 1+q V_{a}-q \psi-E g_{S i} / 2\right) / k_{B} T\right)
\end{aligned}
$$

Boundary conditions:

a. B1: $\psi=0$

b. B2: Surface charge

$$
\sigma_{s}=\left(S B H 1-q \psi-E g_{S i} / 2\right) D_{i t}+D_{f}
$$


c. B3: Surface charge

$$
\sigma_{s}=\left(S B H 1+q V_{a}-q \psi-E g_{S i} / 2\right) D_{i t}+D_{f}
$$

d. $\mathrm{B} 4: \psi=(\mathrm{SBH} 1-\mathrm{SBH} 2) / q+V_{a}$.

4. $\psi$ is the potential of the vacuum level. So the conduction band minimum $E c=q\left(\psi-\chi_{\mathrm{Si}}\right)$ in regions $\mathrm{R} 1$ and $\mathrm{R} 3$, and $E c=q\left(\psi-\chi_{\mathrm{ox}}\right)$ in the region $\mathrm{R} 2$. And the valence band maximum $E v=\left(q \psi-q \chi_{\mathrm{Si}}-E g_{S i}\right)$ in regions $\mathrm{R} 1$ and $\mathrm{R} 3$, and $E v=\left(q \psi-q \chi_{\mathrm{ox}}-E g_{o x}\right)$ in the region $\mathrm{R} 2$.

Vacumm level $q \psi$

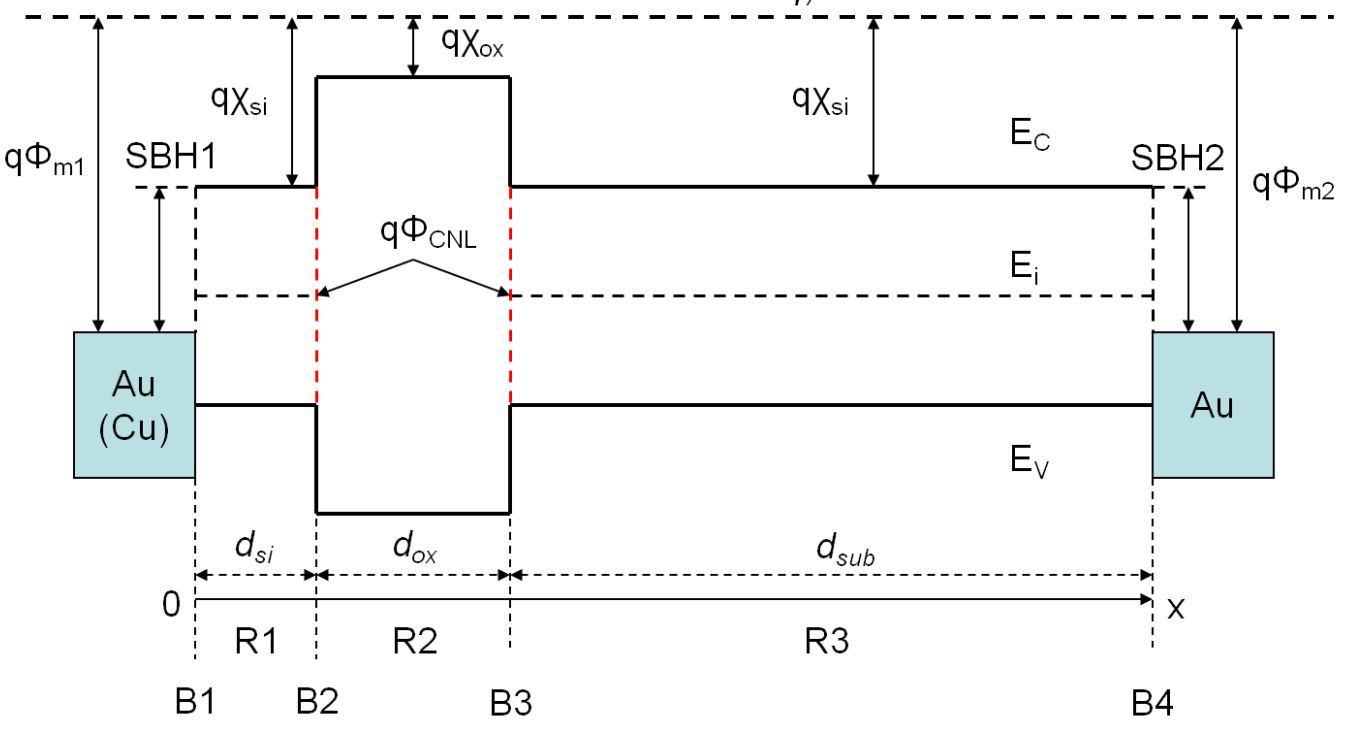

Figure A.1: Geometry for COMSOL numerical simulation. 


\section{REFERENCES}

[1] J.Appenzeller, J.Knoch, V.Derycke, R.Martel, S.Wind, and Ph.Avouris, Phys. Rev. Lett. 89, 126801 (2002).

[2] H.-C. Lin, K.-L.Yeh, T.-Y.Huang, R.-G.Huang, and S.M.Sze, IEEE Trans. Electron Devices 49, 264 (2002).

[3] W.Y.Choi, B.-G.Park, J.D.Lee, and T.-J.King Liu, IEEE Electron Device Lett., 28, 743 (2007).

[4] J.Knoch and J.Appenzeller, Phys. Stat. Sol. (A) 205, 679 (2008).

[5] N. W. Ashcroft and N. D. Mermin, Solid State Physics, Brooks/Cole Thomson Learing, 1976.

[6] S.M.Sze, Physics of Semiconductor Devices, $2^{\text {nd }}$ Ed, Wiley, New York, 1981.

[7] www.wiredchemist.com/chemistry/instructional/intro_chem_valencebond.

[8] D.G.Thomas,ed., II-VI Semiconducting Compounds, W.A.Benjamin, New York, 1967.

[9] YP.Y.Yu and M.Cardona, Fundamentals of Semiconductors - Physics and Materials Properties, $3^{\text {rd }}$ Ed, Springer-Verlag, Berlin, 2001. 
[10] C.A.Hogarth, ed.,Materials Used in Semiconductor Devices, Interscience,New York, 1965.

[11] Y. Tu and J. Tersoff, Phys. Rev. Lett. 84, 4393 (2000).

[12] B.E.Deal, IEE Trans. Electrons Devices 27, 606 (1980).

[13] S.I.Raider and A.Berman, J. Electronchem. Soc.125, 629 (1978).

[14] E.H.Nicollian and J.R.Brews, MOS Physics and Technology, Wiley, New York, 1982.

[15] B.E.Deal, M.Sklar, A.S.Grove, and E.H.Snow, J. Electronchem. Soc.114, 266 (1967).

[16] X.G.Zhang, Electrochemistry of Silicon and its oxide, Kluwer Academic/ Plenum Publishers, New York, 2001.

[17] J.-P. Colinge, Silicon-on-Insulator Technology: Materials to VLSI, $3^{\text {rd }}$ Ed., Springer, New York, 2004.

[18] M.J.Anc, ed., SIMOX, The Institution of Electrical Engineers, London, 2004.

[19] S.Nakashima and K.Izumi, J. Mater. Res. 8, 523 (1993).

[20] S.Nakashima, T.Katayama, Y.Miyamura, A.Matsuzaki, M.Imai, K.Izumi, and N.Ohwada, Proc.IEEE SOI Conf., 71 (1993).

[21] J.Jiao, B.Jonhson, S.Seraphin, M.J.Anc, R.P.Dolan, and B.F.Cordts, Mater. Sci. Eng. B 72, 150 (2000).

[22] A.M.Cowley and S.M.Sze, J. Appl. Phys., 36, 3212 (1965).

[23] D. A. Neamen, Semiconductor Physics and Devices: Basic Principles, Richard D. Irwin, Boston, (1992). 
[24] V.Heine, Phys. Rev., 138, A1689, (1965).

[25] W.E.Spicer, P.W.Chye, P.R.Skeath, C.Y.Su and I.Lindau, J. Vac. Sci. Technol. 16, 1422 (1979).

[26] J.D.Jackson, Classical Electrodynamics, Wiley, New York, 1975.

[27] S.M.Sze, C.R.Crowell, and D.Hahng, J. Appl. Phys., 35, 2534 (1964).

[28] H.A.Bethe, MIT Radiat. Lab. Rep. 43,12 (1942).

[29] C.Y.Chang and S.M.Sze, Solid State Electron., 13, 727 (1970).

[30] F.A.Padovani and R.Stratton, Solid State Electron.,9, 695 (1966).

[31] P.Niedermann, L.Quattropani, K.Solt, A.D.Kent, and Ø.Fischer, J. Vac. Sci. Technol. B, 10, 580 (1992).

[32] H.Palm, M.Arbes and M.Schulz, Phys. Rev. Lett. 71, 2224 (1993).

[33] A.E.Fowell, R.H.William, B.E.Richardson, A.Cafolla, D.I.Westwood, and D.A.Woolf, J. Vac. Sci. Technol. B, 9, 581 (1991).

[34] A.A.Talin, R.S.William, B.A.Morgan, K.M.Ring, and K.L.Kavanagh, J. Vac. Sci. Technol. B, 12, 2634 (1994).

[35] R.T.Tung, Appl. Phys. Lett., 58, 2821 (1991).

[36] R.T.Tung, Phys. Rev. B, 45, 13509 (1992).

[37] W.J.Kaiser and L.D.Bell, Phys. Rev. Lett. 60, 1406 (1988).

[38] T.-H.Shen, M.Eliott, A.E.Fowell, A.Cafolla, B.E.Richardson, D.Westwood and R.H.Williams, J. Vac. Sci. Technol. B 9, 2219 (1991).

[39] C.Tivarus, J.Pelz, M.K.Hudait and S.A.Ringel, Phys. Rev. Lett. 94, 206803 $(2005)$ 
[40] W.H.Rippard and R.A.Buhrman, Phys. Rev. Lett. 84, 971 (2000).

[41] M.T.Cuberes, A.Bauer, H.J.Wen, M.Prietsch, and G.Kaindl, J. Vac. Sci.

Technol. B 12, 2646 (1994).

[42] R.Ludeke, A. Bauer, and E. Cartier, Appl. Phys,Lett. 66, 730 (1995).

[43] B.Kaczer and J.P.Pelz, J. Vac. Sci. Technol. B 14, 2864 (1996).

[44] M.Prietsch, Phys. Rep. 253, 163 (1995).

[45] V.Narayanamurti and M.Kozhevnikov, Phys. Rep. 349, 447 (2001).

[46] Cristian Tivarus, Ph.D Thesis (The Ohio State University, Columbus, 2005).

[47] L.D.Bell and W.J.Kaiser, Phys Rev. Lett. 61, 2368 (1998).

[48] R.Ludeke and M.Prietch, J. Vac. Sci. Technol. A 9, 995 (1991).

[49] R.Ludeke and M.Prietch, Phys. Rev. Lett. 66, 2511 (1991).

[50] J.G.Simmons, J. Appl. Phys. 34, 1793 (1963).

[51] J.A.Stroscio and W.J.Kaier, ed., Scanning Tunneling Microscopy, Academic Press, San Diego, 1993.

[52] E.Y.Lee and L.J.Schowalter, J. Appl. Phys. 70, 2156 (1991).

[53] R.C.Alig, S.Bloom, and C.W.Struck, Phys. Rev. B 22, 5565 (198).

[54] E.Cartier, M.V. Fischetti, E.A.Eklund, and F.R.McFeely, Appl. Phys. Lett. 62, 3339 (1993).

[55] E.A.Eklund, P.D.Kirchner, D.K.Shuh, F.R.McFeely, and E.Cartier, Phys. Rev. Lett. 68, 831 (1992).

[56] Eric Heller, Ph.D Thesis (The Ohio State University, Columbus, 2003).

[57] B. Kaczer, Z. Meng, and J. P. Pelz, Phys. Rev. Lett. 77, 91 (1996). 
[58] http://www.microchem.com

[59] http://snf.stanford.edu/Process/Characterization/NativeOx.html

[60] Benjamin Kaczer, Ph.D thesis, The Ohio State University.

[61] L.D.Bell, W.J.Kaiser, M.H.Hecht, and L.C.Davis, J. Vac. Sci. Technol. B 9, 594 (1991).

[62] L.D.Bell, M.H.Hecht, W.J.Kaiser, and L.C.Davis, Phys. Rev. Lett. 64, 2679 (1990).

[63] M.H.Hecht, L.D.Bell, W.J.Kaiser, and L.C.Davis, Phys. Rev. B 42, 7663 (1990).

[64] E.Y.Lee and L.J.Schowalter, Phys. Rev. B 45, 6325 (1992).

[65] A.Davies and H.G.Craighead, Appl. Phys. Lett. 64, 2833 (1994).

[66] M.T.Cuberes, A.Bauer, H.J.Wen, D.Vandré, M.Prietsch, and G.Kaindl, J. Vac. Sci. Technol. B 12, 2422 (1994).

[67] A.Fernandez, H.D.Hallen, T.Huang, R.A.Huhrman, and J.Silcox, Appl. Phys. Lett. 57, 2826 (1990).

[68] J.J.Garramone, J.R.Abel, I.L.Sitnitsky, R.L.Moore, and V.P.Labella, J. Vac. Sci. Technol.B 27, 2044 (2009).

[69] T.Banerjee, E.Haq, M.H.Siekman, J.C.Lodder and R.Jansen, IEEE Transactions on Magnetics 4, 2642 (2005).

[70] Y.Nakajima et al., Jpn. J. Appl. Phys. 42, 2004 (2003).

[71] X.H. Tang, N.Rechinger, G.Larrieu, E.Dubois, D.Flandre, J-P.Raskin, B.Nysten, A.M.Jonas, and V.Bayot, Nanotechnology 19, 165703 (2008). 
[72] Josh Goldberger, Allon.I.Hochbaum, Rong Fan, and Peidong Yang, Nano Lett. 6, 973 (2006).

[73] Volker Schmidt, Heike Riel, Stephan Senz, Siegfried Karg, Walter Riess, and Ulrich Gösele, Small 2,85 (2006).

[74] Arthur Zhang, Sifang You, Cesare Soci, Yisi Liu, Deli Wang, and Yu-Hwa Lo, Appl.Phys.Lett.93,121110 (2008).

[75] Michael D. Kelzenberg, ShannonW. Boettcher, Jan A. Petykiewicz, Daniel B. Turner-Evans, Morgan C. Putnam, Emily L.Warren, Joshua M. Spurgeon, Ryan M. Briggs, Nathan S. Lewis, and Harry A. Atwater, Nature Materials 9, 239(2010).

[76] J. M.Hergenrother, S.H.Oh, T.Nigam, D.Monroe, F. P Klemens, A.Kornblit, Solid-State Electron.46, 939 (2000).

[77] S.H.Oh, Physics and Technologies of Vertical Transistors. Thesis, Stanford, Palo Alto, CA, 2001.

[78] S.H.Oh, D.Monroe, J.M.Hergenrother, IEEE Electron Device Lett.21, 397 (2000)

[79] A.K.Sharma, S.H.Zaidi, S.Lucero, S.R.JBrueck, N.E.Islam, IEE Proc.sCirc. DeV. Syst. 151, 422 (2004).

[80] F.Leonard and J.Tersoff, Phys.Rev.Lett. 84, 4693 (2000) and Phys.Rev.Lett. 97, 026804 (2006).

[81] R.S.Wagner and W.C.Ellis, Appl.Phys.Lett.4, 89 (1964). 
[82] Brendan M. Kayes,Michael A. Filler,Morgan C. Putnam, Michael D.

Kelzenberg, Nathan S. Lewis, and Harry A. Atwater, Appl.Phys.Lett.91, 103110

(2007).

[83] Y.Wu,Y. Cui, L. Huynh, C. J. Barrelet, D. C. Bell, and

C. M. Lieber, Nano Lett. 4, 433 (2004).

[84] Sergi Gomez and Rodolfo Jun Belen, Mark Kiehlbauch, Eray S. Aydil, J.Vac.Sci.Technol.22,606(2004).

[85] http://www.fei.com/products/dualbeams/helios-nanolab.aspx

[86] J. E. Allen, E. R. Hemesath, D. E. Perea, J. L. Lensch-Falk, Z. Y. Li, F. Yin, M.

H. Gass, P. Wang, A. L. Bleloch, R. E. Palmer, and L. J. Lauhon, Nature Nanotechnology 3, 168 (2008). 